\title{
Atomic data from the Iron Project
}

\section{Relativistic transition probabilities for carbon-like Ar XIII and Fe XXI using Breit-Pauli R-matrix method ${ }^{\star}$}

\author{
S.N. Nahar \\ Department of Astronomy, The Ohio State University, Columbus, OH 43210, U.S.A.
}

Received June 23; accepted September 5, 2000

\begin{abstract}
The Breit-Pauli R-matrix method developed under the Iron Project has been used to obtain extensive sets of oscillator strengths and transition probabilities for dipole allowed and intercombination fine structure transitions in carbon like ions, Ar XIII and Fe XXI. The complete set consists of 1274 fine structure bound energy levels and 198259 oscillator strengths for Ar XIII, and 1611 bound levels and 300079 oscillator strengths for Fe XXI. These correspond to levels of total angular momenta of $0 \leq J \leq 7$ of even and odd parities formed from total spin of $2 S+1=5,3,1$, and orbital angular momenta $0 \leq L \leq 9$ with $n \leq 10,0 \leq l \leq 9$ for each ion. The relativistic effects are included in the Breit-Pauli approximation. The close coupling wavefunction expansion for each ion is represented by the lowest 15 fine structure levels of target configurations, $2 \mathrm{~s}^{2} 2 \mathrm{p}, 2 \mathrm{~s} 2 \mathrm{p}^{2}$ and $2 \mathrm{p}^{3}$. The energy levels are identified spectroscopically using a newly developed identification procedure. The procedure also makes a correspondence between the fine structure energy levels and $L S$ terms. This provides the check for completeness of the calculated levels. Comparison is made of the present energies and the $f$-values with the available observed and theoretical values. Present transition probabilities agree very well with the relativistic atomic structure calculations of Mendoza et al. for the intercombination transitions, $2 \mathrm{~s} 2 \mathrm{p}^{3}\left({ }^{5} \mathrm{~S}_{2}^{\circ}\right)-2 \mathrm{~s}^{2} 2 \mathrm{p}^{2}\left({ }^{3} \mathrm{P}_{1,2},{ }^{1} \mathrm{D}_{2}\right)$. This further indicates that the importance of the neglected Breit interaction decreases with ion charge and constrains the uncertainty in the present calculations to within $15 \%$ even for the weak transitions.
\end{abstract}

Key words: atomic data

\footnotetext{
* Completes tables are only available in electronic form at the CDS via anonymous ftp to cdsarc.u-strasbg.fr (130.79.128.5) or via http://cdsweb.u-strasbg.fr/Abstract.html
}

\section{Introduction}

With the aim of calculating accurate astrophysical opacities, extensive set of oscillator strengths ( $f$-values) for bound - bound transitions and photoionization cross sections for bound - free transitions were obtained under the Opacity Project (The Opacity Project 1995, 1996; Seaton et al. 1994). These data are available through the OP database, the TOPbase (Cunto et al. 1993). Determination of opacities requires transition probabilities or oscillator strengths for all transitions of all ionization stages of the constituent elements in the plasma as obtained under the OP. However, the OP $f$-values were obtained in $L S$ coupling whereas transitions among fine structure levels are often needed in various astrophysical models as well as in experimental or observational spectral analysis. For example, electron densities in solar flares can be determined from the fine structure transitions in Fe XXI (e.g. Pallavicini et al. 1977). Transition probabilies for fine structure levels were obtained for a number of Fe ions, such as Fe II (Nahar 1995), Fe III (Nahar \& Pradhan 1996) and Fe XIII (Nahar 1999) through algebraic transformation of LS multiplets calculated in close coupling approximation. Hence, no relativistic effects were included for these transitions.

The Breit-Pauli R-matrix (BPRM) (Hummer et al. 1993; Berrington et al. 1995) method includes relativistic effects in the Breit-Pauli approximation (Scott \& Burke 1980; Scott \& Taylor 1982). It enables the calculations of both the dipole allowed $(\Delta S=0)$ and the intercombination $(\Delta S \neq 0)$ transitions, in contrast to $L S$ calculations where only dipole allowed transitions could be included. Incorporation of the relativistic effects in the close coupling R-matrix method yields a large number of fine structure transition probabilities with higher accuracy.

However, a major obtstable in the BPRM calculations has been the spectroscopic identification of the large number of fine structure energy levels being calculated. 
These are obtained as the eigenvalues of the BP Hamiltonian labeled only by the total angular momentum and parity, i.e. by $J \pi$, which is insufficient for unique identification. Except for large scale model calculations, complete identification of levels is needed for various diagnostics and spectrocopic applications. A new procedure is developed to identify these levels by a complete set of quantum numbers through analysis of collision channels (Nahar \& Pradhan 2000). The procedure also makes a correspondence between the fine structure levels and their proper $L S$ terms.

BPRM method has been applied for large scale computations of $f$-values for a few iron ions, Fe V (Nahar \& Pradhan 2000; Nahar et al 2000), Fe XXIV and Fe XXV (Nahar \& Pradhan 1999). The $f$-values of Fe XXIV and Fe XXV are found to be very accurate. They agree within a few percent with the measured and other very accurate calculations available for a few transitions. The present work reports oscillator strengths for two carbon like ions, Ar XIII and Fe XXI. Both the dipole allowed and the intercombination transitions are considered. The accuracy and completeness of the results are discussed. While samples of fine structure energy and oscillator strengths are presented herein, the complete tables will be available electronically.

\section{Theory}

In the coupled channel or close coupling (CC) approximation an ion is described in terms of an $(\mathrm{e}+$ ion $)$ complex that comprises of a "target" or core ion, with $N$ bound electrons, and a "free" $(N+1)$-th electron that may be either bound or continuum. For a total spin and angular symmetry $S L \pi$ or $J \pi$, of the $(N+1)$-electron system, the total wavefunction, $\Psi_{\mathrm{E}}$, is represented in terms of the target ion states or levels as:

$\Psi_{\mathrm{E}}(\mathrm{e}+$ ion $)=A \sum_{i} \chi_{i}($ ion $) \theta_{i}+\sum_{j} c_{j} \Phi_{j}$,

where $\chi_{i}$ is the target ion wave function in a specific state $S_{i} L_{i} \pi_{i}$ or level $J_{i} \pi_{i}$, and $\theta_{i}$ is the wave function for the $(N+1)$-th electron in a channel labeled as $S_{i} L_{i}\left(J_{i}\right) \pi_{i} k_{i}^{2} \ell_{i}(S L \pi) \quad[J \pi] ; k_{i}^{2}$ is the incident kinetic energy. In the second sum the $\Phi_{j}$ 's are correlation wavefunctions of the $(N+1)$-electron system that (a) compensate for the orthogonality conditions between the continuum and the bound orbitals, and (b) represent additional short-range correlation that is often of crucial importance in scattering and radiative $\mathrm{CC}$ calculations for each $S L \pi$.

Details of the theory of Breit-Pauli R-matrix method in the close coupling approximation is described in earlier work (Hummer et al. 1993). The method yields the solutions of the relativistic $\mathrm{CC}$ equations using the Breit-Pauli Hamiltonian for the $(N+1)$-electron system to obtain the total wavefunctions $\Psi_{\mathrm{E}}(\mathrm{e}+\mathrm{ion})$. The BreitPauli Hamiltonian is

$H_{N+1}^{\mathrm{BP}}=H_{N+1}+H_{N+1}^{\mathrm{mass}}+H_{N+1}^{\mathrm{Dar}}+H_{N+1}^{\mathrm{so}}$,

where $H_{N+1}$ is the nonrelativistic Hamiltonian,

$H_{N+1}=\sum_{i=1}^{N+1}\left\{-\nabla_{i}^{2}-\frac{2 Z}{r_{i}}+\sum_{j>i}^{N+1} \frac{2}{r_{i j}}\right\}$,

and the relativistic effects are included through the additional terms which are the one-body mass correction term, the Darwin term and the spin-orbit term respectively. The other relatively weaker Breit-interaction,

$H^{\mathrm{B}}=\sum_{i>j}\left[g_{i j}\left(\mathrm{so}+\mathrm{so}^{\prime}\right)+g_{i j}\left(\mathrm{ss}^{\prime}\right)\right]$,

representing the two-body spin-spin and the spin-otherorbit interactions is not included.

The eigenvalues of the $(N+1)$-electron Hamiltonian are the energies of the states such that,

$E=k^{2}>0 \longrightarrow$ continuum (scattering) channel $\left(\Psi_{\mathrm{F}}\right)$

$E=-\frac{z^{2}}{\nu^{2}}<0 \longrightarrow$ bound $\operatorname{state}\left(\Psi_{\mathrm{B}}\right)$,

where $\nu=n-\mu(\ell)$ is the effective quantum number relative to the core level. $\mu(\ell)$ is the quantum defect.

The oscillator strength is proportional to the generalized line strength defined, in either length form or velocity form, by the equations

$S_{\mathrm{L}}=\left|\left\langle\Psi_{k}\left|\sum_{j=1}^{N+1} r_{j}\right| \Psi_{i}\right\rangle\right|^{2}$

and

$S_{\mathrm{V}}=\omega^{-2}\left|\left\langle\Psi_{k}\left|\sum_{j=1}^{N+1} \frac{\partial}{\partial r_{j}}\right| \Psi_{i}\right\rangle\right|^{2}$.

In these equations $\omega$ is the incident photon energy in Rydberg units, and $\Psi_{i}$ and $\Psi_{k}$ are the wave functions representing the initial and final states respectively.

Using the energy difference, $E_{k i}$, between the initial and final states, the oscillator strength, $f_{i k}$, for the transition can be obtained from $S$ as

$f_{i k}=\frac{E_{k i}}{3 g_{i}} S$,

and the Einstein's $A$-coefficient, $A_{k i}$, as

$A_{k i}$ (a.u.) $=\frac{1}{2} \alpha^{3} \frac{g_{i}}{g_{k}} E_{k i}^{2} f_{i k}$,

where $\alpha$ is the fine structure constant, and $g_{i}=2 J_{i}+1$, $g_{k}=2 J_{k}+1$ are the statistical weight factors of the initial and final states, respectively. In terms of c.g.s. unit of time,

$A_{k i}\left(\mathrm{~s}^{-1}\right)=\frac{A_{k i}(\text { a.u. })}{\tau_{0}}$

where $\tau_{0}=2.419^{-17} \mathrm{~s}$ is the atomic unit of time. The lifetime of a level can be obtained from the $A$-values of the level as,

$\tau_{k}=\frac{1}{A_{k}}$ 
where $A_{k}$ is the total radiative transition probability for the level $k$, i.e.,

$A_{k}=\sum_{i} A_{k i}$

\section{Computations}

The R-matrix calculations begin with the target wavefunction obtained through configuration interaction atomic structure calculation. Present target wavefunctions are obtained from the atomic structure calculations using SUPERSTRUCTURE (Eissner et al. 1974). The wavefunctions of Ar XIII and Fe XXI are represented by expansions of 15 fine structure levels of the target or the core ion, Ar XIV and Fe XXII, belonging to the 8 lowest $L S$ terms: $2 \mathrm{~s}^{2} 2 \mathrm{p}\left({ }^{2} \mathrm{P}^{\mathrm{o}}\right), 2 \mathrm{~s} 2 \mathrm{p}^{2}\left({ }^{4} \mathrm{P},{ }^{2} \mathrm{P},{ }^{2} \mathrm{D},{ }^{2} \mathrm{~S}\right)$, and $2 \mathrm{p}^{3}\left({ }^{4} \mathrm{~S}^{\mathrm{o}},{ }^{2} \mathrm{D}^{\mathrm{o}},{ }^{2} \mathrm{P}^{\mathrm{o}}\right)$. The 15 fine structure levels alongwith their energies are listed in Table 1. The energies are the observed ones (Kelly, NIST, for Ar XIV, and Sugar \& Corliss 1985 for Fe XXII) for improved accuray. The correlation and spectroscopic configurations and the values of the scaling parameter $(\lambda)$ in the Thomas-Fermi potential for each orbital of Ar XIV and Fe XXII in the atomic structure calculations are also listed in Table 1. Each target ion was optimized separately with configurations contributing significantly and, hence, does not correspond to the same set. For example, $2 \mathrm{p}^{2} 3$ s does not effect the optimization of Ar XIV, and is not included. The optimization was carried out to achieve close agreement with the observed energies and between the length and velocity $f$-values. The boundchannel term, the second expansion in the wavefunction, Eq. (1), includes all possible configurations upto $2 \mathrm{p}^{4}, 3 \mathrm{~s}^{2}$, $3 \mathrm{p}^{2}$, and $3 \mathrm{~d}^{2}$ for both Ar XIII and Fe XXI.

The one- and two-electron radial integrals are computed by STG1 of the BPRM codes using the one-electron target orbitals generated by SUPERSTRUCTURE. The number of continuum R-matrix basis functions is 12 for each ion. The calculations consider all possible bound levels for $0 \leq J \leq 7$ with $n<10, \ell \leq n-1,0 \leq L \leq 9$, and $(2 S+1)=1,3,5$, even and odd parities. The intermediate coupling calculations are carried out on recoupling the $L S$ symmetries in a pair-coupling representation in stage RECUPD. The (e + core) Hamiltonian matrix is diagonalized for each resulting $J \pi$ in STGH.

STGB of the BPRM codes calculates the fine structure energy levels and their wavefunctions. As fine structure causes large number of closely spaced energy levels, STGB requires to use an energy mesh of effective quantum number, $\Delta \nu=0.001$, an order of magnitude finer than needed in the $L S$ coulping case to avoid levels missing. This increases the computation time considerably.

The oscillator strengths ( $f$-values) are computed using STGBB of the BPRM codes. STGBB computes the transition matrix elements using the bound wavefuncitons created by STGB and angular algebra for the dipole moment calculated by STGH. The STGBB computations are also considerably CPU time extensive due to large number of dipole allowed and intercombination transitions among the fine structure levels.

The BPRM method, which uses the collision theory, describes the energy levels with channel identification for a given total $J \pi$. The information is not adequate for spectroscopic identification of the levels. A level identification procedure as described in Nahar \& Pradhan (2000) is implemented to identify the levels of Ar XIII and Fe XXI. The identification scheme is based on the analysis of quantum defects and percentage weights of the channel wavefunctions similar to that under the Opacity Project (Seaton 1987). Each level is associated with a numer of collision channels. The analysis is carried out over the contributing channels with maximum channel percentage weight, i.e., the dominant channels that determine the proper configurations and terms of the core and the outer electrons. The levels are finally designated with possible identification of $C_{\mathrm{t}}\left(S_{\mathrm{t}} L_{\mathrm{t}} \pi_{\mathrm{t}}\right) J_{\mathrm{t}} n l J(S L) \pi$ where $C_{\mathrm{t}}, S_{\mathrm{t}} L_{\mathrm{t}} \pi_{\mathrm{t}}$, $J_{\mathrm{t}}$ are the configuration, $L S$ term and parity, and total angular momentum of the core or target, $n l$ are the principle and orbital quantum numbers of the outer or the valence electron, $J$ and $S L \pi$ are the total angular momentum, possible $L S$ term and parity of the $(N+1)$-electron system.

The principle quantum number, $n$, of the outer electron of a level is determined from its effective quantum number, $\nu$. For each partial wave $(l), \nu$ of the lowest member (level with the lowest principle quantum number of the valence electron) is determined from quantum defect analysis of all the computed levels with same $l$. The lowest partial wave (e.g. $s$ ) has the highest quantum defect $(\mu)$. A check is maintained to differentiate the quantum defect of a " $s$ " electron from that of an equivalent electron state which has typically a large $\mu$ in a close coupling calculation. Once the lowest $\nu$ is determined, the levels of the corresponding Rydberg series is identified through $\Delta \nu$ which is approximately equal to 1 for the consecutive members. Such pattern for a series can be seen in the complete table for level energies (available electronically). For example, $\nu$ for a $3 s$ electron is about 2.77 and for a $4 s$ electron is about 3.66 for Ar XIII (Table $2 \mathrm{a}, J \pi=0^{\circ}$ ), and it is 2.85 for a $3 s$ electron and 3.85 for a $4 s$ electron for Fe XXI (Table $3 \mathrm{a}, J \pi=0^{\circ}$ ). It may be noted that due to weaker correlation effects quantum defects are more well defined for higher levels, than the lower ones. where the difference, $\Delta \nu$, approaches unity.

Two levels with the same configuration and set of quantum numbers can actually be two independent levels due to outer electron spin addition/subtraction to/from the parent spin angular momentum, i.e. $S_{\mathrm{t}} \pm s=S$. The lower energies are normally assigned with the higher spin multiplicity while the higher levels are with the lower spin multiplicity. However, the energies and effective quantum numbers $(\nu)$ of levels of higher and lower spin multiplicity 
Table 1. The 15 fine structure levels and their relative energies of the target (core) ions Ar XIV and Fe XXII in the close coupling eigenfunction expansions of Ar XIII and Fe XXI. The list of spectroscopic and correlation configurations, and the scaling parameter $(\lambda)$ for each orbital are given below the table

\begin{tabular}{|c|c|c|c|c|c|c|c|c|c|c|c|c|c|c|c|}
\hline \multicolumn{2}{|r|}{ Term } & $\overline{J_{\mathrm{t}}}$ & $E_{\mathrm{t}}(\mathrm{Ry})$ & & Term & $\overline{J_{\mathrm{t}}}$ & $E_{\mathrm{t}}(\mathrm{Ry})$ & & Term & $\overline{J_{\mathrm{t}}}$ & $E_{\mathrm{t}}(\mathrm{Ry})$ & & Term & $J_{\mathrm{t}}$ & $\overline{E_{\mathrm{t}}(\mathrm{Ry})}$ \\
\hline \multicolumn{8}{|c|}{ Ar XIV } & \multicolumn{8}{|c|}{ Fe XXI } \\
\hline 1 & $2 \mathrm{~s}^{2} 2 \mathrm{p}\left({ }^{2} \mathrm{P}^{\mathrm{o}}\right)$ & $1 / 2$ & 0.0 & 9 & $2 \mathrm{~s} 2 \mathrm{p}^{2}\left({ }^{2} \mathrm{P}^{\mathrm{e}}\right)$ & $1 / 2$ & 4.9685 & 1 & $2 \mathrm{~s}^{2} 2 \mathrm{p}\left({ }^{2} \mathrm{P}^{\mathrm{o}}\right)$ & $1 / 2$ & 0.0 & 9 & $2 \mathrm{~s} 2 \mathrm{p}^{2}\left({ }^{2} \mathrm{~S}^{\mathrm{e}}\right)$ & $1 / 2$ & 8.91420 \\
\hline 2 & $2 \mathrm{~s}^{2} 2 \mathrm{p}\left({ }^{2} \mathrm{P}^{\mathrm{o}}\right)$ & $3 / 2$ & 0.20647 & 10 & $2 \mathrm{~s} 2 \mathrm{p}^{2}\left({ }^{2} \mathrm{P}^{\mathrm{e}}\right)$ & $3 / 2$ & 5.0544 & 2 & $2 \mathrm{~s}^{2} 2 \mathrm{p}\left({ }^{2} \mathrm{P}^{\mathrm{o}}\right)$ & $3 / 2$ & 1.07776 & 10 & $2 \mathrm{~s} 2 \mathrm{p}^{2}\left({ }^{2} \mathrm{P}^{\mathrm{e}}\right)$ & $3 / 2$ & 9.04241 \\
\hline 3 & $2 \mathrm{~s} 2 \mathrm{p}^{2}\left({ }^{4} \mathrm{P}^{\mathrm{e}}\right)$ & $1 / 2$ & 1.7925 & 11 & $2 \mathrm{p}^{3}\left({ }^{4} \mathrm{~S}^{\mathrm{O}}\right)$ & $3 / 2$ & 6.2455 & 3 & $2 \mathrm{~s} 2 \mathrm{p}^{2}\left({ }^{4} \mathrm{P}^{\mathrm{e}}\right)$ & $1 / 2$ & 3.68653 & 11 & $2 \mathrm{p}^{3}\left({ }^{4} \mathrm{~S}^{\mathrm{o}}\right)$ & $3 / 2$ & 11.44278 \\
\hline 4 & $2 \mathrm{~s} 2 \mathrm{p}^{2}\left({ }^{4} \mathrm{P}^{\mathrm{e}}\right)$ & $3 / 2$ & 1.8709 & 12 & $2 \mathrm{p}^{3}\left({ }^{2} \mathrm{D}^{\mathrm{o}}\right)$ & $3 / 2$ & 7.1115 & 4 & $2 \mathrm{~s} 2 \mathrm{p}^{2}\left({ }^{4} \mathrm{P}^{\mathrm{e}}\right)$ & $3 / 2$ & 4.19365 & 12 & $2 \mathrm{p}^{3}\left({ }^{2} \mathrm{D}^{\mathrm{o}}\right)$ & $3 / 2$ & 12.72512 \\
\hline 5 & $2 \mathrm{~s} 2 \mathrm{p}^{2}\left({ }^{4} \mathrm{P}^{\mathrm{e}}\right)$ & $5 / 2$ & 1.9756 & 13 & $2 \mathrm{p}^{3}\left({ }^{2} \mathrm{D}^{\mathrm{o}}\right)$ & $5 / 2$ & 7.1361 & 5 & $2 \mathrm{~s} 2 \mathrm{p}^{2}\left({ }^{4} \mathrm{P}^{\mathrm{e}}\right)$ & $5 / 2$ & 4.67717 & 13 & $2 \mathrm{p}^{3}\left({ }^{2} \mathrm{D}^{\mathrm{o}}\right)$ & $5 / 2$ & 13.00269 \\
\hline 6 & $2 \mathrm{~s} 2 \mathrm{p}^{2}\left({ }^{2} \mathrm{D}^{\mathrm{e}}\right)$ & $3 / 2$ & 3.7387 & 14 & $2 \mathrm{p}^{3}\left({ }^{2} \mathrm{P}^{\mathrm{o}}\right)$ & $1 / 2$ & 8.0301 & 6 & $2 \mathrm{~s} 2 \mathrm{p}^{2}\left({ }^{2} \mathrm{D}^{\mathrm{e}}\right)$ & $3 / 2$ & 6.71166 & 14 & $2 \mathrm{p}^{3}\left({ }^{2} \mathrm{P}^{\mathrm{o}}\right)$ & $1 / 2$ & 14.30352 \\
\hline 7 & $2 \mathrm{~s} 2 \mathrm{p}^{2}\left({ }^{2} \mathrm{D}^{\mathrm{e}}\right)$ & $5 / 2$ & 3.7471 & 15 & $2 \mathrm{p}^{3}\left({ }^{2} \mathrm{P}^{\mathrm{o}}\right)$ & $3 / 2$ & 8.0711 & 7 & $2 \mathrm{~s} 2 \mathrm{p}^{2}\left({ }^{2} \mathrm{D}^{\mathrm{e}}\right)$ & $5 / 2$ & 6.92217 & 15 & $2 \mathrm{p}^{3}\left({ }^{2} \mathrm{P}^{\mathrm{o}}\right)$ & $3 / 2$ & 14.83288 \\
\hline 8 & $2 \mathrm{~s} 2 \mathrm{p}^{2}\left({ }^{2} \mathrm{~S}^{\mathrm{e}}\right)$ & $1 / 2$ & 4.6878 & & & & & 8 & $2 \mathrm{~s} 2 \mathrm{p}^{2}\left({ }^{2} \mathrm{P}^{\mathrm{e}}\right)$ & $1 / 2$ & 7.77748 & & & & \\
\hline
\end{tabular}

Spectroscopic: $2 \mathrm{~s}^{2} 2 \mathrm{p}, 2 \mathrm{~s} 2 \mathrm{p}^{2}, 2 \mathrm{p}^{3}$

Correlation: $2 \mathrm{~s}^{2} 3 \mathrm{~s}, 2 \mathrm{~s}^{2} 3 \mathrm{p}, 2 \mathrm{~s}^{2} 3 \mathrm{~d}, 2 \mathrm{~s} 3 \mathrm{p}^{2}, 2 \mathrm{~s} 2 \mathrm{p} 3 \mathrm{~s}, 2 \mathrm{~s} 2 \mathrm{p} 3 \mathrm{p}, 2 \mathrm{~s} 2 \mathrm{p} 3 \mathrm{~d}, 2 \mathrm{p}^{2} 3 \mathrm{p}, 2 \mathrm{p}^{2} 3 \mathrm{~d}, 2 \mathrm{p} 3 \mathrm{p} 3 \mathrm{~d}$

$\lambda: 2.45(1 \mathrm{~s}), 1.34(2 \mathrm{~s}), 1.44(2 \mathrm{p}),-0.76(3 \mathrm{~s}),-0.83(3 \mathrm{p}),-1.5(3 \mathrm{~d})$

Fe XXI:

Spectroscopic: $2 \mathrm{~s}^{2} 2 \mathrm{p}, 2 \mathrm{~s} 2 \mathrm{p}^{2}, 2 \mathrm{p}^{3}$

Correlation: $2 \mathrm{~s}^{2} 3 \mathrm{~s}, 2 \mathrm{~s}^{2} 3 \mathrm{p}, 2 \mathrm{~s}^{2} 3 \mathrm{~d}, 2 \mathrm{~s} 3 \mathrm{~d}^{2}, 2 \mathrm{~s} 2 \mathrm{p} 3 \mathrm{~s}, 2 \mathrm{~s} 2 \mathrm{p} 3 \mathrm{p}, 2 \mathrm{~s} 2 \mathrm{p} 3 \mathrm{~d}, 2 \mathrm{p}^{2} 3 \mathrm{~s}, 2 \mathrm{p}^{2} 3 \mathrm{p}, 2 \mathrm{p}^{2} 3 \mathrm{~d}$

$\lambda: 1.38259(1 \mathrm{~s}), 1.16738(2 \mathrm{~s}), 1.09097(2 \mathrm{p}),-1.18466(3 \mathrm{~s}),-1.18082(3 \mathrm{p}),-1.50447(3 \mathrm{~d})$

can be very close to each other, in which case the observation may show them otherwise.

Following level identification, a direct correspondence is made with standard spectroscopic designations that follow different coupling schemes, such as between $L S$ and $J J$. The correspondence provides the check for completeness of calculated set of levels or the levels missing. A computer program, PRCBPID, is developed to carry out the identification scheme (Nahar \& Pradhan 2000).

The level identification procedure involves considerable manipulation of the bound level data and, although it has been encoded for general applications, still requires analysis and interpretation of problem cases of highly mixed levels that are difficult to identify. The mixed states are dominated by a number of channels. The uncertainty in their identification can be large.

\section{Results and discussions}

The fine structure energy levels and oscillator strengths for the dipole allowed and the intercombination transitions are discussed in separate subsections below.

\subsection{Energy levels}

A total of 1274 bound fine structure energy levels of Ar XIII and 1611 levels of Fe XXI are obtained. These correspond to total angular momenta of $0 \leq J \leq 7$ of both even and odd parities formed from spin multiplicities of $2 S+1=5,3,1$, and total orbital angular momenta of $0 \leq L \leq 9$ with $n \leq 10$, $0 \leq l \leq(n-1)$. The levels are presented in two formats: (i) in $J \pi$ order for practical applications and (ii) in $L S$ term order for spectroscopy and completeness check up.

Tables $2 \mathrm{a}$ and $3 \mathrm{a}$ present a partial set of energy levels of Ar XIII and Fe XXI, respectively, in format (i), i.e., in $J \pi$ order (the complete tables are available electronically). At the top of each set. $N_{J}$ is the total number of energy levels for symmetry of $J \pi$. For example, there are 56 fine structure levels of Ar XIII with $J \pi=0^{\mathrm{e}}$. However, in the table, only part of the levels belonging to symmetry $J \pi$ is presented for illustration. The levels are identified with the configuration and $L S$ term of the core and the outer electron quantum numbers. The effective quantum number, $\left.\nu=z / \sqrt{(} E-E_{\mathrm{t}}\right)$ where $E_{\mathrm{t}}$ is the immediate target threshold, is given next to the energies. However, $\nu$ is not given for the equivalent electron levels as it is not defined for these levels. Each level is assigned to one or more $L S$ terms in the last column. If number of possible term is more than one, all are specified. However, the proper term from a multiple possibilities can be determined from Hund's rule; the term with higher angular momentum lies lower in energy. For example, the three levels 4, 5, and 6 of set $J \pi=1^{\mathrm{e}}$ in Table $2 \mathrm{a}$ are assigned with 3 possible terms, ${ }^{3}(S P D)^{\mathrm{e}}$, whereupon the first level, i.e., level 4 can be designated as the ${ }^{3} \mathrm{D}^{\mathrm{e}}$, while level 5 is ${ }^{3} \mathrm{P}^{\mathrm{e}}$, and level 6 is ${ }^{3} \mathrm{~S}$. One reason for specifying all possible terms is that the order of calculated energy levels may not match exactly that of the measured ones. The other reason is that Hund's rule may not apply to all cases for complex ions; nonetheless it is useful to establish completeness. Similar sets of levels for Fe XXI are presented in Table 3a.

Tables $2 \mathrm{~b}$ and $3 \mathrm{~b}$ present the energy levels of Ar XIII and Fe XXI, respectively, in ascending order regardless of $J \pi$ values, and are grouped together according to the 
Table 2. a. Calculated BPRM fine structure energy levels of Ar XIII with spectrocopic identification. $N_{J}=$ total number of levels of symmetry $J \pi$. $S L \pi$ lists possible set of $L S$ terms

\begin{tabular}{|c|c|c|c|c|c|c|c|c|}
\hline$i$ & $C_{\mathrm{t}}$ & $S_{\mathrm{t}} L_{\mathrm{t}} \pi_{\mathrm{t}}$ & $J_{\mathrm{t}}$ & $n l$ & $J$ & $E(\mathrm{Ry})$ & $\nu$ & $S L \pi$ \\
\hline \multicolumn{9}{|c|}{$N_{J}=56, \quad J \pi=0^{\mathrm{e}}$} \\
\hline 1 & $2 \mathrm{~s} 22 \mathrm{p} 2$ & & & & 0 & $-5.04159 \mathrm{E}+01$ & & ${ }^{3} \mathrm{Pe}$ \\
\hline 2 & $2 \mathrm{~s} 22 \mathrm{p} 2$ & & & & 0 & $-4.89054 \mathrm{E}+01$ & & ${ }^{1} \mathrm{Se}$ \\
\hline 3 & $2 \mathrm{p} 4$ & & & & 0 & $-4.14331 \mathrm{E}+01$ & & ${ }^{3} \mathrm{Pe}$ \\
\hline 4 & $2 \mathrm{p} 4$ & & & & 0 & $-3.96913 \mathrm{E}+01$ & & ${ }^{1} \mathrm{Se}$ \\
\hline 5 & $2 \mathrm{~s} 22 \mathrm{p}$ & ${ }^{2} \mathrm{P}^{\mathrm{o}}$ & $1 / 2$ & $3 p$ & 0 & $-2.05345 \mathrm{E}+01$ & 2.87 & ${ }^{3} \mathrm{Pe}$ \\
\hline 6 & $2 \mathrm{~s} 22 \mathrm{p}$ & ${ }^{2} \mathrm{P}^{\mathrm{o}}$ & $3 / 2$ & $3 p$ & 0 & $-1.97019 \mathrm{E}+01$ & 2.91 & ${ }^{1} \mathrm{Se}$ \\
\hline 7 & $2 \mathrm{~s} 2 \mathrm{p} 2$ & ${ }^{4} \mathrm{P}^{\mathrm{e}}$ & $1 / 2$ & $3 \mathrm{~s}$ & 0 & $-1.93963 \mathrm{E}+01$ & 2.82 & ${ }^{3} \mathrm{Pe}$ \\
\hline 8 & $2 \mathrm{~s} 2 \mathrm{p} 2$ & ${ }^{4} \mathrm{P}^{\mathrm{e}}$ & $3 / 2$ & $3 \mathrm{~d}$ & 0 & $-1.74680 \mathrm{E}+01$ & 2.96 & ${ }^{5} \mathrm{De}$ \\
\hline 9 & $2 \mathrm{~s} 2 \mathrm{p} 2$ & ${ }^{4} \mathrm{P}^{\mathrm{e}}$ & $5 / 2$ & $3 \mathrm{~d}$ & 0 & $-1.71405 \mathrm{E}+01$ & 2.97 & ${ }^{3} \mathrm{Pe}$ \\
\hline 10 & $2 \mathrm{~s} 2 \mathrm{p} 2$ & ${ }^{2} \mathrm{~S}^{\mathrm{e}}$ & $1 / 2$ & $3 \mathrm{~s}$ & 0 & $-1.70355 \mathrm{E}+01$ & 2.79 & ${ }^{1} \mathrm{Se}$ \\
\hline 11 & $2 \mathrm{~s} 2 \mathrm{p} 2$ & ${ }^{2} \mathrm{P}^{\mathrm{e}}$ & $1 / 2$ & $3 \mathrm{~s}$ & 0 & $-1.67478 \mathrm{E}+01$ & 2.79 & ${ }^{3} \mathrm{Pe}$ \\
\hline 12 & $2 \mathrm{~s} 2 \mathrm{p} 2$ & ${ }^{2} \mathrm{D}^{\mathrm{e}}$ & $3 / 2$ & $3 \mathrm{~d}$ & 0 & $-1.53551 \mathrm{E}+01$ & 2.97 & ${ }^{3} \mathrm{Pe}$ \\
\hline 13 & $2 \mathrm{~s} 2 \mathrm{p} 2$ & ${ }^{2} \mathrm{D}^{\mathrm{e}}$ & $5 / 2$ & $3 \mathrm{~d}$ & 0 & $-1.49520 \mathrm{E}+01$ & 3.01 & ${ }^{1} \mathrm{Se}$ \\
\hline 14 & $2 \mathrm{~s} 2 \mathrm{p} 2$ & ${ }^{2} \mathrm{P}^{\mathrm{e}}$ & $3 / 2$ & $3 \mathrm{~d}$ & 0 & $-1.41433 \mathrm{E}+01$ & 2.97 & ${ }^{3} \mathrm{Pe}$ \\
\hline 15 & $2 \mathrm{p} 3$ & ${ }^{4} \mathrm{~S}^{\circ}$ & $3 / 2$ & $3 p$ & 0 & $-1.36805 \mathrm{E}+01$ & 2.91 & ${ }^{3} \mathrm{Pe}$ \\
\hline 16 & $2 \mathrm{~s} 22 \mathrm{p}$ & ${ }^{2} \mathrm{P}^{\mathrm{o}}$ & $1 / 2$ & $4 \mathrm{p}$ & 0 & $-1.31449 \mathrm{E}+01$ & 3.59 & ${ }^{3} \mathrm{Pe}$ \\
\hline \multicolumn{9}{|c|}{$N_{J}=53, \quad J \pi=0^{\circ}$} \\
\hline 1 & $2 \mathrm{~s} 2 \mathrm{p} 3$ & & & & 0 & $-4.58522 \mathrm{E}+01$ & & ${ }^{3} \mathrm{Po}$ \\
\hline 2 & $2 \mathrm{~s} 22 \mathrm{p}$ & ${ }^{2} \mathrm{P}^{\mathrm{o}}$ & $1 / 2$ & $3 \mathrm{~s}$ & 0 & $-2.20575 \mathrm{E}+01$ & 2.77 & ${ }^{3} \mathrm{Po}$ \\
\hline 3 & $2 \mathrm{~s} 22 \mathrm{p}$ & ${ }^{2} \mathrm{P}^{\mathrm{o}}$ & $3 / 2$ & $3 \mathrm{~d}$ & 0 & $-1.90794 \mathrm{E}+01$ & 2.96 & ${ }^{3} \mathrm{Po}$ \\
\hline 4 & $2 \mathrm{~s} 2 \mathrm{p} 2$ & ${ }^{4} \mathrm{P}^{\mathrm{e}}$ & $1 / 2$ & $3 p$ & 0 & $-1.86737 \mathrm{E}+01$ & 2.87 & ${ }^{5} \mathrm{Do}$ \\
\hline 5 & $2 \mathrm{~s} 2 \mathrm{p} 2$ & ${ }^{4} \mathrm{P}^{\mathrm{e}}$ & $3 / 2$ & $3 p$ & 0 & $-1.80112 \mathrm{E}+01$ & 2.91 & ${ }^{3} \mathrm{Po}$ \\
\hline 6 & $2 \mathrm{~s} 2 \mathrm{p} 2$ & ${ }^{2} \mathrm{D}^{\mathrm{e}}$ & $3 / 2$ & $3 p$ & 0 & $-1.64710 \mathrm{E}+01$ & 2.89 & ${ }^{3} \mathrm{Po}$ \\
\hline 7 & $2 \mathrm{~s} 2 \mathrm{p} 2$ & ${ }^{2} \mathrm{~S}^{\mathrm{e}}$ & $1 / 2$ & $3 p$ & 0 & $-1.59211 \mathrm{E}+01$ & 2.86 & ${ }^{3} \mathrm{Po}$ \\
\hline 8 & $2 \mathrm{~s} 2 \mathrm{p} 2$ & ${ }^{2} \mathrm{P}^{\mathrm{e}}$ & $3 / 2$ & $3 p$ & 0 & $-1.57212 \mathrm{E}+01$ & 2.85 & ${ }^{3} \mathrm{Po}$ \\
\hline 9 & $2 \mathrm{~s} 2 \mathrm{p} 2$ & ${ }^{2} \mathrm{P}^{\mathrm{e}}$ & $1 / 2$ & $3 p$ & 0 & $-1.54559 \mathrm{E}+01$ & 2.88 & ${ }^{1}$ So \\
\hline 10 & $2 \mathrm{p} 3$ & ${ }^{2} \mathrm{P}^{\mathrm{o}}$ & $1 / 2$ & $3 \mathrm{~s}$ & 0 & $-1.33819 \mathrm{E}+01$ & 2.81 & ${ }^{3} \mathrm{Po}$ \\
\hline 11 & $2 \mathrm{p} 3$ & ${ }^{4} \mathrm{~S}^{\circ}$ & $3 / 2$ & $3 \mathrm{~d}$ & 0 & $-1.30580 \mathrm{E}+01$ & 2.96 & ${ }^{5} \mathrm{Do}$ \\
\hline 12 & $2 \mathrm{~s} 22 \mathrm{p}$ & ${ }^{2} \mathrm{P}^{\mathrm{o}}$ & $1 / 2$ & $4 \mathrm{~s}$ & 0 & $-1.26019 \mathrm{E}+01$ & 3.66 & ${ }^{3} \mathrm{Po}$ \\
\hline 13 & $2 \mathrm{p} 3$ & ${ }^{2} \mathrm{D}^{\mathrm{o}}$ & $5 / 2$ & $3 \mathrm{~d}$ & 0 & $-1.21319 \mathrm{E}+01$ & 2.96 & ${ }^{3} \mathrm{Po}$ \\
\hline 14 & $2 \mathrm{p} 3$ & ${ }^{2} \mathrm{D}^{\mathrm{o}}$ & $3 / 2$ & $3 \mathrm{~d}$ & 0 & $-1.17041 \mathrm{E}+01$ & 3.00 & ${ }^{1}$ So \\
\hline 15 & $2 \mathrm{~s} 2 \mathrm{p} 2$ & ${ }^{4} \mathrm{P}^{\mathrm{e}}$ & $1 / 2$ & $4 \mathrm{p}$ & 0 & $-1.14540 \mathrm{E}+01$ & 3.57 & ${ }^{5} \mathrm{Do}$ \\
\hline 16 & $2 \mathrm{~s} 2 \mathrm{p} 2$ & ${ }^{4} \mathrm{P}^{\mathrm{e}}$ & $3 / 2$ & $4 \mathrm{p}$ & 0 & $-1.11632 \mathrm{E}+01$ & 3.60 & ${ }^{3} \mathrm{Po}$ \\
\hline \multicolumn{9}{|c|}{$N_{J}=131, \quad J \pi=1^{\mathrm{e}}$} \\
\hline 1 & $2 \mathrm{~s} 22 \mathrm{p} 2$ & & & & 1 & $-5.03260 \mathrm{E}+01$ & & ${ }^{3} \mathrm{Pe}$ \\
\hline 2 & $2 \mathrm{p} 4$ & & & & 1 & $-4.14770 \mathrm{E}+01$ & & ${ }^{3} \mathrm{Pe}$ \\
\hline 3 & $2 \mathrm{~s} 22 \mathrm{p}$ & ${ }^{2} \mathrm{P}^{\mathrm{o}}$ & $1 / 2$ & $3 p$ & 1 & $-2.08448 \mathrm{E}+01$ & 2.85 & ${ }^{1} \mathrm{Pe}$ \\
\hline 4 & $2 \mathrm{~s} 22 \mathrm{p}$ & ${ }^{2} \mathrm{P}^{\mathrm{o}}$ & $3 / 2$ & $3 p$ & 1 & $-2.07028 \mathrm{E}+01$ & 2.84 & ${ }^{3} \mathrm{SPDe}$ \\
\hline 5 & $2 \mathrm{~s} 22 \mathrm{p}$ & ${ }^{2} \mathrm{P}^{\mathrm{o}}$ & $3 / 2$ & $3 p$ & 1 & $-2.05068 \mathrm{E}+01$ & 2.86 & ${ }^{3} \mathrm{SPDe}$ \\
\hline 6 & $2 \mathrm{~s} 22 \mathrm{p}$ & ${ }^{2} \mathrm{P}^{\mathrm{o}}$ & $1 / 2$ & $3 p$ & 1 & $-2.04340 \mathrm{E}+01$ & 2.88 & ${ }^{3} \mathrm{SPDe}$ \\
\hline 7 & $2 \mathrm{~s} 2 \mathrm{p} 2$ & ${ }^{4} \mathrm{P}^{\mathrm{e}}$ & $1 / 2$ & $3 \mathrm{~s}$ & 1 & $-1.99821 \mathrm{E}+01$ & 2.79 & ${ }^{5} \mathrm{Pe}$ \\
\hline 8 & $2 \mathrm{~s} 2 \mathrm{p} 2$ & ${ }^{4} \mathrm{P}^{\mathrm{e}}$ & $3 / 2$ & $3 \mathrm{~s}$ & 1 & $-1.93348 \mathrm{E}+01$ & 2.82 & ${ }^{3} \mathrm{Pe}$ \\
\hline 9 & $2 \mathrm{~s} 2 \mathrm{p} 2$ & ${ }^{2} \mathrm{D}^{\mathrm{e}}$ & $3 / 2$ & $3 \mathrm{~s}$ & 1 & $-1.81570 \mathrm{E}+01$ & 2.78 & ${ }^{3} \mathrm{De}$ \\
\hline 10 & $2 \mathrm{~s} 2 \mathrm{p} 2$ & ${ }^{4} \mathrm{P}^{\mathrm{e}}$ & $1 / 2$ & $3 \mathrm{~d}$ & 1 & $-1.76801 \mathrm{E}+01$ & 2.95 & ${ }^{5} \mathrm{PDFe}$ \\
\hline 11 & $2 \mathrm{~s} 2 \mathrm{p} 2$ & ${ }^{4} \mathrm{P}^{\mathrm{e}}$ & $3 / 2$ & $3 \mathrm{~d}$ & 1 & $-1.74609 \mathrm{E}+01$ & 2.96 & ${ }^{5} \mathrm{PDFe}$ \\
\hline 12 & $2 \mathrm{~s} 2 \mathrm{p} 2$ & ${ }^{4} \mathrm{Pe}^{\mathrm{e}}$ & $5 / 2$ & $3 \mathrm{~d}$ & 1 & $-1.72186 \mathrm{E}+01$ & 2.97 & ${ }^{5} \mathrm{PDFe}$ \\
\hline 13 & $2 \mathrm{~s} 2 \mathrm{p} 2$ & ${ }^{4} \mathrm{P}^{\mathrm{e}}$ & $5 / 2$ & $3 \mathrm{~d}$ & 1 & $-1.71594 \mathrm{E}+01$ & 2.97 & ${ }^{3} \mathrm{PDe}$ \\
\hline 14 & $2 \mathrm{~s} 2 \mathrm{p} 2$ & ${ }^{4} \mathrm{P}^{\mathrm{e}}$ & $5 / 2$ & $3 \mathrm{~d}$ & 1 & $-1.71434 \mathrm{E}+01$ & 2.97 & ${ }^{3} \mathrm{PDe}$ \\
\hline 15 & $2 \mathrm{~s} 2 \mathrm{p} 2$ & ${ }^{2} \mathrm{~S}^{\mathrm{e}}$ & $1 / 2$ & $3 \mathrm{~s}$ & 1 & $-1.69176 \mathrm{E}+01$ & 2.80 & ${ }^{3} \mathrm{Se}$ \\
\hline 16 & $2 \mathrm{~s} 2 \mathrm{p} 2$ & ${ }^{2} \mathrm{P}^{\mathrm{e}}$ & $3 / 2$ & $3 \mathrm{~s}$ & 1 & $-1.67240 \mathrm{E}+01$ & 2.79 & ${ }^{3} \mathrm{Pe}$ \\
\hline \multicolumn{9}{|c|}{$N_{J}=136, \quad J \pi=1^{\circ}$} \\
\hline 1 & $2 \mathrm{~s} 2 \mathrm{p} 3$ & & & & 1 & $-4.65272 \mathrm{E}+01$ & & ${ }^{3} \mathrm{Do}$ \\
\hline 2 & 2s2p3 & & & & 1 & $-4.58436 \mathrm{E}+01$ & & ${ }^{3} \mathrm{Po}$ \\
\hline 3 & $2 \mathrm{~s} 2 \mathrm{p} 3$ & & & & 1 & $-4.45972 \mathrm{E}+01$ & & ${ }^{3}$ So \\
\hline 4 & $2 \mathrm{~s} 2 \mathrm{p} 3$ & & & & 1 & $-4.39490 \mathrm{E}+01$ & & ${ }^{1} \mathrm{Po}$ \\
\hline 5 & $2 \mathrm{~s} 22 \mathrm{p}$ & ${ }^{2} \mathrm{P}^{\mathrm{o}}$ & $3 / 2$ & $3 \mathrm{~s}$ & 1 & $-2.20006 \mathrm{E}+01$ & 2.76 & ${ }^{3} \mathrm{Po}$ \\
\hline 6 & $2 \mathrm{~s} 22 \mathrm{p}$ & ${ }^{2} \mathrm{P}^{\mathrm{o}}$ & $1 / 2$ & $3 \mathrm{~s}$ & 1 & $-2.17248 \mathrm{E}+01$ & 2.79 & ${ }^{1} \mathrm{Po}$ \\
\hline 7 & $2 \mathrm{~s} 22 \mathrm{p}$ & ${ }^{2} \mathrm{P}^{\mathrm{o}}$ & $1 / 2$ & $3 \mathrm{~d}$ & 1 & $-1.92584 \mathrm{E}+01$ & 2.96 & ${ }^{3} \mathrm{PDo}$ \\
\hline 8 & $2 \mathrm{~s} 22 \mathrm{p}$ & ${ }^{2} \mathrm{P}^{\mathrm{o}}$ & $3 / 2$ & $3 \mathrm{~d}$ & 1 & $-1.91117 \mathrm{E}+01$ & 2.96 & ${ }^{3} \mathrm{PDo}$ \\
\hline 9 & $2 \mathrm{~s} 22 \mathrm{p}$ & ${ }^{2} \mathrm{P}^{\mathrm{o}}$ & $3 / 2$ & $3 \mathrm{~d}$ & 1 & $-1.87724 \mathrm{E}+01$ & 2.98 & ${ }^{1} \mathrm{Po}$ \\
\hline 10 & $2 \mathrm{~s} 2 \mathrm{p} 2$ & ${ }^{4} \mathrm{P}^{\mathrm{e}}$ & $3 / 2$ & $3 p$ & 1 & $-1.86813 \mathrm{E}+01$ & 2.87 & ${ }^{3} \mathrm{SPDo}$ \\
\hline 11 & $2 \mathrm{~s} 2 \mathrm{p} 2$ & ${ }^{4} \mathrm{P}^{\mathrm{e}}$ & $1 / 2$ & $3 p$ & 1 & $-1.86458 \mathrm{E}+01$ & 2.88 & ${ }^{5} \mathrm{PDo}$ \\
\hline 12 & $2 \mathrm{~s} 2 \mathrm{p} 2$ & ${ }^{4} \mathrm{P}^{\mathrm{e}}$ & $3 / 2$ & $3 p$ & 1 & $-1.84751 \mathrm{E}+01$ & 2.88 & ${ }^{5} \mathrm{PDo}$ \\
\hline 13 & $2 \mathrm{~s} 2 \mathrm{p} 2$ & ${ }^{4} \mathrm{P}^{\mathrm{e}}$ & $1 / 2$ & $3 p$ & 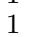 & $-1.82444 \mathrm{E}+01$ & 2.90 & ${ }^{3} \mathrm{SPDo}$ \\
\hline 14 & $2 \mathrm{~s} 2 \mathrm{p} 2$ & ${ }^{4} \mathrm{P}^{\mathrm{e}}$ & $1 / 2$ & $3 p$ & 1 & $-1.79813 \mathrm{E}+01$ & 2.92 & ${ }^{3} \mathrm{SPDo}$ \\
\hline 15 & $2 \mathrm{~s} 2 \mathrm{p} 2$ & ${ }^{2} \mathrm{D}^{\mathrm{e}}$ & $3 / 2$ & $3 p$ & 1 & $-1.66700 \mathrm{E}+01$ & 2.88 & ${ }^{1} \mathrm{Po}$ \\
\hline 16 & $2 \mathrm{~s} 2 \mathrm{p} 2$ & ${ }^{2} \mathrm{D}^{\mathrm{e}}$ & $5 / 2$ & $3 p$ & 1 & $-1.66046 \mathrm{E}+01$ & 2.88 & ${ }^{3} \mathrm{PDo}$ \\
\hline
\end{tabular}


Table 2. b. Calculated BPRM fine strucuture energy levels of Ar XIII, identified and ordered. Nlv = total number of levels expected for the possible set of $L S$ terms listed and $N l v(\mathrm{c})=$ number of calculated levels. $S L \pi$ lists the possible $L S$ terms for each level. (See texts for details)

\begin{tabular}{|c|c|c|c|c|c|c|c|}
\hline$C_{\mathrm{t}}$ & $S_{\mathrm{t}} L_{\mathrm{t}} \pi_{\mathrm{t}}$ & $J_{\mathrm{t}}$ & $n l$ & $J$ & $E(\mathrm{cal})$ & $\nu$ & $S L \pi$ \\
\hline $\begin{array}{l}\text { Eqv electron/u } \\
2 \mathrm{p} 4 \\
2 \mathrm{p} 4 \\
2 \mathrm{p} 4 \\
N l v(\mathrm{c})=3 \text { : set }\end{array}$ & $\begin{array}{l}\text { identified } \\
\text { complete }\end{array}$ & levels, & parity & $\begin{array}{l}: \mathrm{e} \\
2 \\
1 \\
0\end{array}$ & $\begin{array}{l}-4.16174 \mathrm{E}+01 \\
-4.14770 \mathrm{E}+01 \\
-4.14331 \mathrm{E}+01\end{array}$ & & 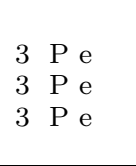 \\
\hline $\begin{array}{l}N l v=3, \quad 3, \mathrm{o}: \\
2 \mathrm{~s} 22 \mathrm{p} \\
2 \mathrm{~s} 22 \mathrm{p} \\
2 \mathrm{~s} 22 \mathrm{p} \\
\operatorname{Nlv}(\mathrm{c})=3: \mathrm{s}\end{array}$ & $\begin{array}{l}\mathrm{P}(210 \\
(2 \mathrm{Po}) \\
(2 \mathrm{Po}) \\
(2 \mathrm{Po}) \\
\text { complete }\end{array}$ & $\begin{array}{l}) \\
1 / 2 \\
3 / 2 \\
3 / 2\end{array}$ & $\begin{array}{l}3 \mathrm{~s} \\
3 \mathrm{~s} \\
3 \mathrm{~s}\end{array}$ & $\begin{array}{l}0 \\
1 \\
2\end{array}$ & $\begin{array}{l}-2.20575 \mathrm{E}+01 \\
-2.20006 \mathrm{E}+01 \\
-2.18454 \mathrm{E}+01\end{array}$ & $\begin{array}{l}2.77 \\
2.76 \\
2.77\end{array}$ & $\begin{array}{lll}3 & \mathrm{P} & \mathrm{o} \\
3 & \mathrm{P} & \mathrm{o} \\
3 & \mathrm{P} & \mathrm{o}\end{array}$ \\
\hline $\begin{array}{l}N l v=1,1, \mathrm{o}: \\
2 \mathrm{~s} 22 \mathrm{p} \\
N l v(c)=1: \mathrm{set}\end{array}$ & $\begin{array}{c}\mathrm{P}(1) \\
(2 \mathrm{Po}) \\
\text { complete }\end{array}$ & $1 / 2$ & $3 \mathrm{~s}$ & 1 & $-2.17248 \mathrm{E}+01$ & 2.79 & $1 \mathrm{P}$ o \\
\hline $\begin{array}{l}N l v=3, \quad 1, \mathrm{e}: \\
2 \mathrm{~s} 22 \mathrm{p} \\
2 \mathrm{~s} 22 \mathrm{p} \\
2 \mathrm{~s} 22 \mathrm{p} \\
\operatorname{Nlv}(\mathrm{c})=3: \mathrm{s}\end{array}$ & $\begin{array}{l}\mathrm{S}(0) \mathrm{P} \\
(2 \mathrm{Po}) \\
(2 \mathrm{Po}) \\
(2 \mathrm{Po}) \\
\text { complete }\end{array}$ & $\begin{array}{l}(1) \\
1 / 2 \\
3 / 2 \\
3 / 2 \\
\end{array}$ & $\begin{array}{l}(2) \\
3 \mathrm{p} \\
3 \mathrm{p} \\
3 \mathrm{p}\end{array}$ & $\begin{array}{l}1 \\
2 \\
0\end{array}$ & $\begin{array}{l}-2.08448 \mathrm{E}+01 \\
-2.06875 \mathrm{E}+01 \\
-1.97019 \mathrm{E}+01\end{array}$ & $\begin{array}{l}2.85 \\
2.84 \\
2.91\end{array}$ & $\begin{array}{ll}1 & \mathrm{P} \mathrm{e} \\
1 & \mathrm{D} \mathrm{e} \\
1 & \mathrm{~S} \mathrm{e}\end{array}$ \\
\hline $\begin{array}{l}N l v=7,3, \mathrm{e}: \\
2 \mathrm{~s} 22 \mathrm{p} \\
2 \mathrm{~s} 22 \mathrm{p} \\
2 \mathrm{~s} 22 \mathrm{p} \\
2 \mathrm{~s} 22 \mathrm{p} \\
2 \mathrm{~s} 22 \mathrm{p} \\
2 \mathrm{~s} 22 \mathrm{p} \\
2 \mathrm{~s} 22 \mathrm{p} \\
N l v(c)=7: \text { set }\end{array}$ & $\begin{array}{c}\mathrm{S}(1) \mathrm{P} \\
(2 \mathrm{Po}) \\
(2 \mathrm{Po}) \\
(2 \mathrm{Po}) \\
(2 \mathrm{Po}) \\
(2 \mathrm{Po}) \\
(2 \mathrm{Po}) \\
(2 \mathrm{Po}) \\
\text { complete } \\
\end{array}$ & $\begin{array}{l}(21 \\
3 / 2 \\
3 / 2 \\
1 / 2 \\
3 / 2 \\
1 / 2 \\
3 / 2 \\
3 / 2\end{array}$ & $\begin{array}{l}7 \mathrm{D} \\
3 \mathrm{p} \\
3 \mathrm{p} \\
3 \mathrm{p} \\
3 \mathrm{p} \\
3 \mathrm{p} \\
3 \mathrm{p} \\
3 \mathrm{p}\end{array}$ & $\begin{array}{l}32 \\
1 \\
3 \\
0 \\
1 \\
1 \\
2 \\
2\end{array}$ & $\begin{array}{l}1 \text { ) } \\
-2.07028 \mathrm{E}+01 \\
-2.05487 \mathrm{E}+01 \\
-2.05345 \mathrm{E}+01 \\
-2.05068 \mathrm{E}+01 \\
-2.04340 \mathrm{E}+01 \\
-2.03885 \mathrm{E}+01 \\
-2.00647 \mathrm{E}+01 \\
\end{array}$ & $\begin{array}{l}2.84 \\
2.85 \\
2.87 \\
2.86 \\
2.88 \\
2.86 \\
2.89\end{array}$ & $\begin{array}{ll}3 & \text { SPD e } \\
3 & \mathrm{D} \mathrm{e} \\
3 & \mathrm{P} \mathrm{e} \\
3 & \mathrm{SPD} \text { e } \\
3 & \text { SPD e } \\
3 & \mathrm{PD} \mathrm{e} \\
3 & \mathrm{PD} \mathrm{e}\end{array}$ \\
\hline $\begin{array}{l}N l v=3, \quad 5, \mathrm{e}: \\
2 \mathrm{~s} 2 \mathrm{p} 2 \\
2 \mathrm{~s} 2 \mathrm{p} 2 \\
2 \mathrm{~s} 2 \mathrm{p} 2 \\
N l v(c)=3: \text { set }\end{array}$ & 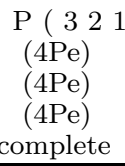 & $\begin{array}{l}) \\
1 / 2 \\
3 / 2 \\
5 / 2\end{array}$ & $\begin{array}{l}3 \mathrm{~s} \\
3 \mathrm{~s} \\
3 \mathrm{~s}\end{array}$ & $\begin{array}{l}1 \\
2 \\
3\end{array}$ & $\begin{array}{l}-1.99821 \mathrm{E}+01 \\
-1.99038 \mathrm{E}+01 \\
-1.98012 \mathrm{E}+01\end{array}$ & $\begin{array}{l}2.79 \\
2.79 \\
2.79\end{array}$ & $\begin{array}{ll}5 & \mathrm{P} \mathrm{e} \\
5 & \mathrm{P} \mathrm{e} \\
5 & \mathrm{P} \mathrm{e}\end{array}$ \\
\hline $\begin{array}{l}N l v=3, \quad 1, \mathrm{o}: \\
2 \mathrm{~s} 22 \mathrm{p} \\
2 \mathrm{~s} 22 \mathrm{p} \\
2 \mathrm{~s} 22 \mathrm{p} \\
N l v(\mathrm{c})=3: \text { set }\end{array}$ & $\begin{array}{c}\mathrm{P}(\mathrm{l}) \mathrm{D} \\
(2 \mathrm{Po}) \\
(2 \mathrm{Po}) \\
(2 \mathrm{Po}) \\
\text { complete }\end{array}$ & $\begin{array}{l}(2) \\
1 / 2 \\
3 / 2 \\
3 / 2\end{array}$ & $\begin{array}{l}F(3) \\
3 d \\
3 d \\
3 d\end{array}$ & $\begin{array}{l}2 \\
3 \\
1\end{array}$ & $\begin{array}{l}-1.95754 \mathrm{E}+01 \\
-1.87869 \mathrm{E}+01 \\
-1.87724 \mathrm{E}+01\end{array}$ & $\begin{array}{l}2.94 \\
2.98 \\
2.98\end{array}$ & $\begin{array}{lll}1 & \mathrm{D} & \mathrm{o} \\
1 & \mathrm{~F} & \mathrm{o} \\
1 & \mathrm{P} & \mathrm{o}\end{array}$ \\
\hline $\begin{array}{l}N l v=9,3, \mathrm{o}: \\
2 \mathrm{~s} 22 \mathrm{p} \\
2 \mathrm{~s} 22 \mathrm{p} \\
2 \mathrm{~s} 22 \mathrm{p} \\
2 \mathrm{~s} 22 \mathrm{p} \\
2 \mathrm{~s} 22 \mathrm{p} \\
2 \mathrm{~s} 22 \mathrm{p} \\
2 \mathrm{~s} 22 \mathrm{p} \\
2 \mathrm{~s} 22 \mathrm{p} \\
2 \mathrm{~s} 22 \mathrm{p} \\
N l v(\mathrm{c})=9: \mathrm{se}\end{array}$ & $\begin{array}{l}\mathrm{P}(2 \mathrm{ll} 0 \\
(2 \mathrm{Po}) \\
(2 \mathrm{Po}) \\
(2 \mathrm{Po}) \\
(2 \mathrm{Po}) \\
(2 \mathrm{Po}) \\
(2 \mathrm{Po}) \\
(2 \mathrm{Po}) \\
(2 \mathrm{Po}) \\
(2 \mathrm{Po}) \\
\text { complete }\end{array}$ & $\begin{array}{l}\text { ) } \mathrm{D}( \\
1 / 2 \\
3 / 2 \\
3 / 2 \\
1 / 2 \\
3 / 2 \\
3 / 2 \\
3 / 2 \\
3 / 2 \\
3 / 2 \\
\end{array}$ & $\begin{array}{l}321 \\
3 \mathrm{~d} \\
3 \mathrm{~d} \\
3 \mathrm{~d} \\
3 \mathrm{~d} \\
3 \mathrm{~d} \\
3 \mathrm{~d} \\
3 \mathrm{~d} \\
3 \mathrm{~d} \\
3 \mathrm{~d}\end{array}$ & $\begin{array}{l}\mathrm{F} \\
3 \\
2 \\
4 \\
1 \\
2 \\
3 \\
2 \\
1 \\
0\end{array}$ & $\begin{array}{l}432.9) \\
-1.94634 \mathrm{E}+01 \\
-1.94284 \mathrm{E}+01 \\
-1.93579 \mathrm{E}+01 \\
-1.92584 \mathrm{E}+01 \\
-1.92077 \mathrm{E}+01 \\
-1.91768 \mathrm{E}+01 \\
-1.91492 \mathrm{E}+01 \\
-1.91117 \mathrm{E}+01 \\
-1.90794 \mathrm{E}+01\end{array}$ & $\begin{array}{l}2.95 \\
2.93 \\
2.94 \\
2.96 \\
2.95 \\
2.95 \\
2.95 \\
2.96 \\
2.96 \\
\end{array}$ & $\begin{array}{ll}3 & \text { DF o } \\
3 & \text { PDF o } \\
3 & \text { F o } \\
3 & \text { PD o } \\
3 & \text { PDF o } \\
3 & \text { DF o } \\
3 & \text { PDF o } \\
3 & \text { PD o } \\
3 & \text { P o }\end{array}$ \\
\hline $\begin{array}{l}N l v=9, \quad 3, \mathrm{e} \\
2 \mathrm{~s} 22 \mathrm{p} \\
2 \mathrm{~s} 22 \mathrm{p} \\
2 \mathrm{~s} 22 \mathrm{p} \\
2 \mathrm{~s} 22 \mathrm{p} \\
2 \mathrm{~s} 22 \mathrm{p} \\
N l v(\mathrm{c})=5, N l\end{array}$ & $\begin{array}{l}\mathrm{G}(543 \\
(2 \mathrm{Po}) \\
(2 \mathrm{Po}) \\
(2 \mathrm{Po}) \\
(2 \mathrm{Po}) \\
(2 \mathrm{Po}) \\
=9: \text { set } \mathrm{i} \\
\end{array}$ & $\begin{array}{l}3) \mathrm{H}( \\
1 / 2 \\
1 / 2 \\
1 / 2 \\
3 / 2 \\
3 / 2 \\
\text { incomp }\end{array}$ & $\begin{array}{l}654 \\
6 \mathrm{~h} \\
6 \mathrm{~h} \\
6 \mathrm{~h} \\
6 \mathrm{~h} \\
6 \mathrm{~h} \\
\text { lete, }\end{array}$ & $\begin{array}{c}\text { I }( \\
4 \\
5 \\
6 \\
7 \\
3 \\
\text { evel }\end{array}$ & $\begin{array}{l}765) \\
-4.69457 \mathrm{E}+00 \\
-4.69457 \mathrm{E}+00 \\
-4.69455 \mathrm{E}+00 \\
-4.48800 \mathrm{E}+00 \\
-4.48795 \mathrm{E}+00 \\
\text { nissing: } 5465\end{array}$ & $\begin{array}{l}6.00 \\
6.00 \\
6.00 \\
6.00 \\
6.00\end{array}$ & $\begin{array}{ll}3 & \mathrm{GH} \mathrm{e} \\
3 & \mathrm{GHI} \text { e } \\
3 & \mathrm{HI} \mathrm{e} \\
3 & \mathrm{I} \mathrm{e} \\
3 & \mathrm{G} \mathrm{e}\end{array}$ \\
\hline $\begin{array}{l}N l v=3, \quad 1, \mathrm{o}: \\
2 \mathrm{~s} 22 \mathrm{p} \\
2 \mathrm{~s} 22 \mathrm{p} \\
2 \mathrm{~s} 22 \mathrm{p} \\
N l v(\mathrm{c})=3: \mathrm{set}\end{array}$ & $\begin{array}{c}\mathrm{P}(1) \mathrm{D} \\
(2 \mathrm{Po}) \\
(2 \mathrm{Po}) \\
(2 \mathrm{Po}) \\
\text { complete }\end{array}$ & $\begin{array}{l}(2) \\
3 / 2 \\
3 / 2 \\
3 / 2\end{array}$ & $\begin{array}{l}(3) \\
6 \mathrm{~d} \\
6 \mathrm{~d} \\
6 \mathrm{~d}\end{array}$ & $\begin{array}{l}2 \\
3 \\
1\end{array}$ & $\begin{array}{l}-4.58862 \mathrm{E}+00 \\
-4.54791 \mathrm{E}+00 \\
-4.54451 \mathrm{E}+00\end{array}$ & $\begin{array}{l}5.94 \\
5.96 \\
5.96\end{array}$ & $\begin{array}{lll}1 & \mathrm{D} & \mathrm{o} \\
1 & \mathrm{~F} & \mathrm{o} \\
1 & \mathrm{P} & \mathrm{o}\end{array}$ \\
\hline
\end{tabular}


Table 3. a. Calculated BPRM fine structure energy levels of Fe XXI with spectrocopic identification. $N_{J}=$ total number of levels of symmetry $J \pi . S L \pi$ lists possible set of $L S$ terms

\begin{tabular}{|c|c|c|c|c|c|c|c|c|}
\hline$i$ & $C_{\mathrm{t}}$ & $S_{\mathrm{t}} L_{\mathrm{t}} \pi_{\mathrm{t}}$ & $J_{\mathrm{t}}$ & $n l$ & $J$ & $E(\mathrm{Ry})$ & $\nu$ & $S L \pi$ \\
\hline & & & \multicolumn{2}{|c|}{$N_{J}=64}$, & \multicolumn{2}{|c|}{$J \pi=0^{\mathrm{e}}$} & & \\
\hline 1 & $2 \mathrm{~s} 22 \mathrm{p} 2$ & & & & 0 & $-1.24292 \mathrm{E}+02$ & & ${ }^{3} \mathrm{Pe}$ \\
\hline 2 & $2 \mathrm{~s} 22 \mathrm{p} 2$ & & & & 0 & $-1.20849 \mathrm{E}+02$ & & ${ }^{1} \mathrm{Se}$ \\
\hline 3 & $2 \mathrm{p} 4$ & & & & 0 & $-1.08406 \mathrm{E}+02$ & & ${ }^{3} \mathrm{Pe}$ \\
\hline 4 & $2 \mathrm{p} 4$ & & & & 0 & $-1.05511 \mathrm{E}+02$ & & ${ }^{1} \mathrm{Se}$ \\
\hline 5 & $2 \mathrm{~s} 22 \mathrm{p}$ & ${ }^{2} \mathrm{P}^{\mathrm{o}}$ & $1 / 2$ & $3 p$ & 0 & $-5.21862 \mathrm{E}+01$ & 2.91 & ${ }^{3} \mathrm{Pe}$ \\
\hline 6 & $2 \mathrm{~s} 2 \mathrm{p} 2$ & ${ }^{4} \mathrm{P}^{\mathrm{e}}$ & $1 / 2$ & $3 s$ & 0 & $-5.03119 \mathrm{E}+01$ & 2.86 & ${ }^{3} \mathrm{Pe}$ \\
\hline 7 & $2 \mathrm{~s} 22 \mathrm{p}$ & ${ }^{2} \mathrm{P}^{\mathrm{o}}$ & $3 / 2$ & $3 \mathrm{p}$ & 0 & $-4.99106 \mathrm{E}+01$ & 2.94 & ${ }^{1} \mathrm{Se}$ \\
\hline 8 & $2 \mathrm{~s} 2 \mathrm{p} 2$ & ${ }^{2} \mathrm{P}^{\mathrm{e}}$ & $1 / 2$ & $3 \mathrm{~s}$ & 0 & $-4.66313 \mathrm{E}+01$ & 2.85 & ${ }^{3} \mathrm{Pe}$ \\
\hline 9 & $2 \mathrm{~s} 2 \mathrm{p} 2$ & ${ }^{4} \mathrm{P}^{\mathrm{e}}$ & $3 / 2$ & $3 \mathrm{~d}$ & 0 & $-4.62816 \mathrm{E}+01$ & 2.96 & ${ }^{5} \mathrm{De}$ \\
\hline 10 & $2 \mathrm{~s} 2 \mathrm{p} 2$ & ${ }^{2} \mathrm{Se}^{\mathrm{e}}$ & $1 / 2$ & $3 \mathrm{~s}$ & 0 & $-4.52695 \mathrm{E}+01$ & 2.85 & ${ }^{1} \mathrm{Se}$ \\
\hline 11 & $2 \mathrm{~s} 2 \mathrm{p} 2$ & ${ }^{4} \mathrm{Pe}^{\mathrm{e}}$ & $5 / 2$ & $3 d$ & 0 & $-4.52395 \mathrm{E}+01$ & 2.97 & ${ }^{3} \mathrm{Pe}$ \\
\hline 12 & $2 \mathrm{~s} 2 \mathrm{p} 2$ & ${ }^{2} \mathrm{D}^{\mathrm{e}}$ & $3 / 2$ & $3 d$ & 0 & $-4.30183 \mathrm{E}+01$ & 2.98 & ${ }^{3} \mathrm{Pe}$ \\
\hline 13 & $2 \mathrm{~s} 2 \mathrm{p} 2$ & ${ }^{2} \mathrm{D}^{\mathrm{e}}$ & $5 / 2$ & $3 \mathrm{~d}$ & 0 & $-4.22934 \mathrm{E}+01$ & 2.99 & ${ }^{1} \mathrm{Se}$ \\
\hline 14 & $2 \mathrm{~s} 2 \mathrm{p} 2$ & ${ }^{2} \mathrm{P}^{\mathrm{e}}$ & $3 / 2$ & $3 \mathrm{~d}$ & 0 & $-4.08816 \mathrm{E}+01$ & 2.97 & ${ }^{3} \mathrm{Pe}$ \\
\hline 15 & $2 \mathrm{p} 3$ & ${ }^{4} \mathrm{~S}^{\mathrm{O}}$ & $3 / 2$ & $3 p$ & 0 & $-4.01067 \mathrm{E}+01$ & 2.92 & ${ }^{3} \mathrm{Pe}$ \\
\hline \multirow[t]{2}{*}{16} & $2 \mathrm{p} 3$ & ${ }^{2} \mathrm{D}^{\mathrm{o}}$ & $3 / 2$ & $3 \mathrm{p}$ & 0 & $-3.85891 \mathrm{E}+01$ & 2.93 & ${ }^{3} \mathrm{Pe}$ \\
\hline & & & \multicolumn{2}{|c|}{$N_{J}=61}$, & \multicolumn{2}{|c|}{$J \pi=0^{\circ}$} & & \\
\hline 1 & $2 \mathrm{~s} 2 \mathrm{p} 3$ & & & & 0 & $-1.15895 \mathrm{E}+02$ & & ${ }^{3} \mathrm{Po}$ \\
\hline 2 & $2 \mathrm{~s} 22 \mathrm{p}$ & ${ }^{2} \mathrm{P}^{\mathrm{o}}$ & $1 / 2$ & $3 \mathrm{~s}$ & 0 & $-5.48009 \mathrm{E}+01$ & 2.84 & ${ }^{3} \mathrm{Po}$ \\
\hline 3 & $2 \mathrm{~s} 22 \mathrm{p}$ & ${ }^{2} \mathrm{P}^{\mathrm{o}}$ & $3 / 2$ & $3 d$ & 0 & $-4.90612 \mathrm{E}+01$ & 2.97 & ${ }^{3} \mathrm{Po}$ \\
\hline 4 & $2 \mathrm{~s} 2 \mathrm{p} 2$ & ${ }^{4} \mathrm{P}^{\mathrm{e}}$ & $1 / 2$ & $3 \mathrm{p}$ & 0 & $-4.89298 \mathrm{E}+01$ & 2.89 & ${ }^{5} \mathrm{Do}$ \\
\hline 5 & $2 \mathrm{~s} 2 \mathrm{p} 2$ & ${ }^{4} \mathrm{P}^{\mathrm{e}}$ & $3 / 2$ & $3 p$ & 0 & $-4.73308 \mathrm{E}+01$ & 2.93 & ${ }^{3} \mathrm{Po}$ \\
\hline 6 & $2 \mathrm{~s} 2 \mathrm{p} 2$ & ${ }^{2} \mathrm{D}^{\mathrm{e}}$ & $3 / 2$ & $3 \mathrm{p}$ & 0 & $-4.50107 \mathrm{E}+01$ & 2.92 & ${ }^{3} \mathrm{Po}$ \\
\hline 7 & $2 \mathrm{~s} 2 \mathrm{p} 2$ & ${ }^{2} \mathrm{Pe}^{\mathrm{e}}$ & $1 / 2$ & $3 p$ & 0 & $-4.46865 \mathrm{E}+01$ & 2.90 & ${ }^{3} \mathrm{Po}$ \\
\hline 8 & $2 \mathrm{~s} 2 \mathrm{p} 2$ & ${ }^{2} \mathrm{Pe}^{\mathrm{e}}$ & $3 / 2$ & $3 \mathrm{p}$ & 0 & $-4.35887 \mathrm{E}+01$ & 2.89 & ${ }^{1}$ So \\
\hline 9 & $2 \mathrm{~s} 2 \mathrm{p} 2$ & ${ }^{2} \mathrm{~S}^{\mathrm{e}}$ & $1 / 2$ & $3 \mathrm{p}$ & 0 & $-4.31686 \mathrm{E}+01$ & 2.91 & ${ }^{3} \mathrm{Po}$ \\
\hline 10 & $2 \mathrm{p} 3$ & ${ }^{2} \mathrm{P}^{\mathrm{o}}$ & $1 / 2$ & $3 \mathrm{~s}$ & 0 & $-3.98818 \mathrm{E}+01$ & 2.85 & ${ }^{3} \mathrm{Po}$ \\
\hline 11 & $2 \mathrm{p} 3$ & ${ }^{4} \mathrm{~S}^{\mathrm{o}}$ & $3 / 2$ & $3 \mathrm{~d}$ & 0 & $-3.89417 \mathrm{E}+01$ & 2.96 & ${ }^{5} \mathrm{Do}$ \\
\hline 12 & $2 \mathrm{p} 3$ & ${ }^{2} \mathrm{D}^{\circ}$ & $3 / 2$ & $3 \mathrm{~d}$ & 0 & $-3.74513 \mathrm{E}+01$ & 2.96 & ${ }^{3} \mathrm{Po}$ \\
\hline 13 & $2 \mathrm{p} 3$ & ${ }^{2} \mathrm{D}^{\mathrm{o}}$ & $5 / 2$ & $3 \mathrm{~d}$ & 0 & $-3.66926 \mathrm{E}+01$ & 2.98 & ${ }^{1}$ So \\
\hline 14 & $2 \mathrm{p} 3$ & ${ }^{2} \mathrm{P}^{\mathrm{o}}$ & $3 / 2$ & $3 \mathrm{~d}$ & 0 & $-3.53176 \mathrm{E}+01$ & 2.96 & ${ }^{3} \mathrm{Po}$ \\
\hline 15 & $2 \mathrm{~s} 22 \mathrm{p}$ & ${ }^{2} \mathrm{P}^{\mathrm{o}}$ & $1 / 2$ & $4 \mathrm{~s}$ & 0 & $-2.97182 \mathrm{E}+01$ & 3.85 & ${ }^{3} \mathrm{Po}$ \\
\hline \multirow[t]{2}{*}{16} & $2 \mathrm{~s} 22 \mathrm{p}$ & ${ }^{2} \mathrm{P}^{\mathrm{o}}$ & $3 / 2$ & $4 \mathrm{~d}$ & 0 & $-2.69365 \mathrm{E}+01$ & 3.97 & ${ }^{3} \mathrm{Po}$ \\
\hline & & & \multicolumn{2}{|c|}{$N_{J}=153}$, & \multicolumn{2}{|c|}{$J \pi=1^{\mathrm{e}}$} & & \\
\hline 1 & $2 \mathrm{~s} 22 \mathrm{p} 2$ & & & & 1 & $-1.23615 \mathrm{E}+02$ & & ${ }^{3} \mathrm{Pe}$ \\
\hline 2 & $2 \mathrm{p} 4$ & & & & 1 & $-1.08363 \mathrm{E}+02$ & & ${ }^{3} \mathrm{Pe}$ \\
\hline 3 & $2 \mathrm{~s} 22 \mathrm{p}$ & ${ }^{2} \mathrm{P}^{\mathrm{o}}$ & $1 / 2$ & $3 p$ & 1 & $-5.28040 \mathrm{E}+01$ & 2.89 & ${ }^{3}$ SPDe \\
\hline 4 & $2 \mathrm{~s} 22 \mathrm{p}$ & ${ }^{2} \mathrm{P}^{\mathrm{o}}$ & $1 / 2$ & $3 \mathrm{p}$ & 1 & $-5.23926 \mathrm{E}+01$ & 2.90 & ${ }^{3} \mathrm{SPDe}$ \\
\hline 5 & $2 \mathrm{~s} 22 \mathrm{p}$ & ${ }^{2} \mathrm{P}^{\mathrm{o}}$ & $3 / 2$ & $3 p$ & 1 & $-5.15048 \mathrm{E}+01$ & 2.90 & ${ }^{1} \mathrm{Pe}$ \\
\hline 6 & $2 \mathrm{~s} 22 \mathrm{p}$ & ${ }^{2} \mathrm{P}^{\mathrm{o}}$ & $3 / 2$ & $3 \mathrm{p}$ & 1 & $-5.13231 \mathrm{E}+01$ & 2.90 & ${ }^{3} \mathrm{SPDe}$ \\
\hline 7 & $2 \mathrm{~s} 2 \mathrm{p} 2$ & ${ }^{4} \mathrm{P}^{\mathrm{e}}$ & $1 / 2$ & $3 \mathrm{~s}$ & 1 & $-5.09584 \mathrm{E}+01$ & 2.84 & ${ }^{5} \mathrm{Pe}$ \\
\hline 8 & $2 \mathrm{~s} 2 \mathrm{p} 2$ & ${ }^{4} \mathrm{P}^{\mathrm{e}}$ & $3 / 2$ & $3 \mathrm{~s}$ & 1 & $-4.96425 \mathrm{E}+01$ & 2.86 & ${ }^{3} \mathrm{Pe}$ \\
\hline 9 & $2 \mathrm{~s} 2 \mathrm{p} 2$ & ${ }^{2} \mathrm{D}^{\mathrm{e}}$ & $3 / 2$ & $3 \mathrm{~s}$ & 1 & $-4.78489 \mathrm{E}+01$ & 2.84 & ${ }^{3} \mathrm{De}$ \\
\hline 10 & $2 \mathrm{~s} 2 \mathrm{p} 2$ & ${ }^{4} \mathrm{P}^{\mathrm{e}}$ & $1 / 2$ & $3 \mathrm{~d}$ & 1 & $-4.68999 \mathrm{E}+01$ & 2.95 & ${ }^{5} \mathrm{PDFe}$ \\
\hline 11 & $2 \mathrm{~s} 2 \mathrm{p} 2$ & ${ }^{2} \mathrm{~S}^{\mathrm{e}}$ & $1 / 2$ & $3 \mathrm{~s}$ & 1 & $-4.65180 \mathrm{E}+01$ & 2.82 & ${ }^{3} \mathrm{Se}$ \\
\hline 12 & $2 \mathrm{~s} 2 \mathrm{p} 2$ & ${ }^{4} \mathrm{P}^{\mathrm{e}}$ & $3 / 2$ & $3 \mathrm{~d}$ & 1 & $-4.62583 \mathrm{E}+01$ & 2.96 & ${ }^{5} \mathrm{PDFe}$ \\
\hline 13 & $2 \mathrm{~s} 2 \mathrm{p} 2$ & ${ }^{2} \mathrm{P}^{\mathrm{e}}$ & $1 / 2$ & $3 \mathrm{~s}$ & 1 & $-4.58680 \mathrm{E}+01$ & 2.87 & ${ }^{3} \mathrm{Pe}$ \\
\hline 14 & $2 \mathrm{~s} 2 \mathrm{p} 2$ & ${ }^{4} \mathrm{P}^{\mathrm{e}}$ & $5 / 2$ & $3 \mathrm{~d}$ & 1 & $-4.55835 \mathrm{E}+01$ & 2.96 & ${ }^{5} \mathrm{PDFe}$ \\
\hline 15 & $2 \mathrm{~s} 2 \mathrm{p} 2$ & ${ }^{2} \mathrm{P}^{\mathrm{e}}$ & $3 / 2$ & $3 \mathrm{~s}$ & 1 & $-4.53921 \mathrm{E}+01$ & 2.85 & ${ }^{1} \mathrm{Pe}$ \\
\hline \multirow[t]{2}{*}{16} & $2 \mathrm{~s} 2 \mathrm{p} 2$ & ${ }^{4} \mathrm{P}^{\mathrm{e}}$ & $5 / 2$ & $3 \mathrm{~d}$ & 1 & $-4.52726 \mathrm{E}+01$ & 2.97 & ${ }^{3} \mathrm{PDe}$ \\
\hline & & & \multicolumn{2}{|c|}{$N_{J}=157$} & & $\pi=1^{\circ}$ & & \\
\hline 1 & $2 \mathrm{~s} 2 \mathrm{p} 3$ & & & & 1 & $-1.17168 \mathrm{E}+02$ & & ${ }^{3} \mathrm{Do}$ \\
\hline 2 & $2 \mathrm{~s} 2 \mathrm{p} 3$ & & & & 1 & $-1.15815 \mathrm{E}+02$ & & ${ }^{3} \mathrm{Po}$ \\
\hline 3 & $2 \mathrm{~s} 2 \mathrm{p} 3$ & & & & 1 & $-1.14241 \mathrm{E}+02$ & & ${ }^{3}$ So \\
\hline 4 & $2 \mathrm{~s} 2 \mathrm{p} 3$ & & & & 1 & $-1.12695 \mathrm{E}+02$ & & ${ }^{1} \mathrm{Po}$ \\
\hline 5 & $2 \mathrm{~s} 22 \mathrm{p}$ & ${ }^{2} \mathrm{P}^{\mathrm{o}}$ & $3 / 2$ & $3 \mathrm{~s}$ & 1 & $-5.45579 \mathrm{E}+01$ & 2.81 & ${ }^{3} \mathrm{Po}$ \\
\hline 6 & $2 \mathrm{~s} 22 \mathrm{p}$ & ${ }^{2} \mathrm{P}^{\mathrm{o}}$ & $1 / 2$ & $3 \mathrm{~s}$ & 1 & $-5.36838 \mathrm{E}+01$ & 2.87 & ${ }^{1} \mathrm{Po}$ \\
\hline 7 & $2 \mathrm{~s} 22 \mathrm{p}$ & ${ }^{2} \mathrm{P}^{\mathrm{o}}$ & $1 / 2$ & $3 d$ & 1 & $-4.98528 \mathrm{E}+01$ & 2.97 & ${ }^{3} \mathrm{PDo}$ \\
\hline 8 & $2 \mathrm{~s} 22 \mathrm{p}$ & ${ }^{2} \mathrm{Po}^{\mathrm{o}}$ & $3 / 2$ & $3 \mathrm{~d}$ & 1 & $-4.92676 \mathrm{E}+01$ & 2.96 & ${ }^{3} \mathrm{PDo}$ \\
\hline 9 & $2 \mathrm{~s} 2 \mathrm{p} 2$ & ${ }^{4} \mathrm{P}^{\mathrm{e}}$ & $1 / 2$ & $3 p$ & 1 & $-4.89841 \mathrm{E}+01$ & 2.89 & ${ }^{5} \mathrm{PDo}$ \\
\hline 10 & $2 \mathrm{~s} 2 \mathrm{p} 2$ & ${ }^{4} \mathrm{Pe}^{\mathrm{e}}$ & $1 / 2$ & $3 \mathrm{p}$ & 1 & $-4.87720 \mathrm{E}+01$ & 2.90 & ${ }^{5} \mathrm{PDo}$ \\
\hline 11 & $2 \mathrm{~s} 22 \mathrm{p}$ & ${ }^{2} \mathrm{P}^{\mathrm{o}}$ & $3 / 2$ & $3 d$ & 1 & $-4.84796 \mathrm{E}+01$ & 2.98 & ${ }^{1} \mathrm{Po}$ \\
\hline 12 & $2 \mathrm{~s} 2 \mathrm{p} 2$ & ${ }^{4} \mathrm{P}^{\mathrm{e}}$ & $3 / 2$ & $3 p$ & 1 & $-4.79949 \mathrm{E}+01$ & 2.91 & ${ }^{3} \mathrm{SPDo}$ \\
\hline 13 & $2 \mathrm{~s} 2 \mathrm{p} 2$ & ${ }^{4} \mathrm{P}^{\mathrm{e}}$ & $3 / 2$ & $3 p$ & 1 & $-4.78167 \mathrm{E}+01$ & 2.91 & ${ }^{3} \mathrm{SPDo}$ \\
\hline 14 & $2 \mathrm{~s} 2 \mathrm{p} 2$ & ${ }^{4} \mathrm{P}^{\mathrm{e}}$ & $1 / 2$ & $3 \mathrm{p}$ & 1 & $-4.69872 \mathrm{E}+01$ & 2.95 & ${ }^{3} \mathrm{SPDo}$ \\
\hline 15 & $2 \mathrm{~s} 2 \mathrm{p} 2$ & ${ }^{2} \mathrm{D}^{\mathrm{e}}$ & $3 / 2$ & $3 \mathrm{p}$ & 1 & $-4.57908 \mathrm{E}+01$ & 2.90 & ${ }^{1} \mathrm{Po}$ \\
\hline 16 & $2 \mathrm{~s} 2 \mathrm{p} 2$ & ${ }^{2} \mathrm{D}^{\mathrm{e}}$ & $5 / 2$ & $3 \mathrm{p}$ & 1 & $-4.51108 \mathrm{E}+01$ & 2.91 & ${ }^{3} \mathrm{PDo}$ \\
\hline
\end{tabular}


Table 3. b. Calculated BPRM fine structure energy levels of Fe XXI, identified and ordered. Nlv = total number of levels expected for the possible set of $L S$ terms listed and $N l v(\mathrm{c})=$ number of calculated levels. $S L \pi$ lists the possible $L S$ terms for each level. (See texts for details)

\begin{tabular}{|c|c|c|c|c|c|c|c|}
\hline$C_{\mathrm{t}}$ & $S_{\mathrm{t}} L_{\mathrm{t}} \pi_{\mathrm{t}}$ & $J_{\mathrm{t}}$ & $n l$ & $J$ & $E(\mathrm{cal})$ & $\nu$ & $S L \pi$ \\
\hline $\begin{array}{l}N l v=3, \quad 3, \mathrm{o}: \\
2 \mathrm{~s} 22 \mathrm{p} \\
2 \mathrm{~s} 22 \mathrm{p} \\
2 \mathrm{~s} 22 \mathrm{p} \\
N l v(\mathrm{c})=3: \mathrm{set}\end{array}$ & $\begin{array}{l}\mathrm{P}(210 \\
\text { (2Po) } \\
\text { (2Po) } \\
\text { (2Po) } \\
\text { complete } \\
\end{array}$ & $\begin{array}{l} \\
1 / 2 \\
3 / 2 \\
3 / 2\end{array}$ & $\begin{array}{l}3 \mathrm{~s} \\
3 \mathrm{~s} \\
3 \mathrm{~s}\end{array}$ & $\begin{array}{l}0 \\
1 \\
2\end{array}$ & $\begin{array}{l}-5.48009 \mathrm{E}+01 \\
-5.45579 \mathrm{E}+01 \\
-5.37314 \mathrm{E}+01 \\
\end{array}$ & $\begin{array}{l}2.84 \\
2.81 \\
2.84\end{array}$ & $\begin{array}{lll}3 & \mathrm{P} & \mathrm{o} \\
3 & \mathrm{P} & \mathrm{o} \\
3 & \mathrm{P} & \mathrm{o}\end{array}$ \\
\hline $\begin{array}{l}N l v=1, \quad 1, \mathrm{o}: \\
2 \mathrm{~s} 22 \mathrm{p} \\
N l v(\mathrm{c})=1: \text { set }\end{array}$ & $\begin{array}{c}\mathrm{P}(1) \\
(2 \mathrm{Po}) \\
\text { complete }\end{array}$ & $1 / 2$ & $3 \mathrm{~s}$ & 1 & $-5.36838 \mathrm{E}+01$ & 2.87 & $1 \mathrm{P} \circ$ \\
\hline $\begin{array}{l}N l v=7, \quad 3, \mathrm{e}: \\
2 \mathrm{~s} 22 \mathrm{p} \\
2 \mathrm{~s} 22 \mathrm{p} \\
2 \mathrm{~s} 22 \mathrm{p} \\
2 \mathrm{~s} 22 \mathrm{p} \\
2 \mathrm{~s} 22 \mathrm{p} \\
2 \mathrm{~s} 22 \mathrm{p} \\
2 \mathrm{~s} 22 \mathrm{p} \\
N l v(\mathrm{c})=7 \text { : set }\end{array}$ & $\begin{array}{c}\mathrm{S}(1) \mathrm{P} \\
(2 \mathrm{Po}) \\
(2 \mathrm{Po}) \\
(2 \mathrm{Po}) \\
(2 \mathrm{Po}) \\
(2 \mathrm{Po}) \\
\text { (2Po) } \\
\text { (2Po) } \\
\text { complete }\end{array}$ & $\begin{array}{l}(211 \\
1 / 2 \\
1 / 2 \\
1 / 2 \\
1 / 2 \\
3 / 2 \\
3 / 2 \\
3 / 2\end{array}$ & $\begin{array}{l}7 \mathrm{D} \\
3 \mathrm{p} \\
3 \mathrm{p} \\
3 \mathrm{p} \\
3 \mathrm{p} \\
3 \mathrm{p} \\
3 \mathrm{p} \\
3 \mathrm{p}\end{array}$ & $\begin{array}{l}32 \\
1 \\
1 \\
2 \\
0 \\
3 \\
1 \\
2\end{array}$ & $\begin{array}{l}1 \text { ) } \\
-5.28040 \mathrm{E}+01 \\
-5.23926 \mathrm{E}+01 \\
-5.23595 \mathrm{E}+01 \\
-5.21862 \mathrm{E}+01 \\
-5.14268 \mathrm{E}+01 \\
-5.13231 \mathrm{E}+01 \\
-5.12455 \mathrm{E}+01\end{array}$ & $\begin{array}{l}2.89 \\
2.90 \\
2.90 \\
2.91 \\
2.90 \\
2.90 \\
2.90\end{array}$ & $\begin{array}{ll}3 & \text { SPD e } \\
3 & \text { SPD e } \\
3 & \mathrm{PD} \mathrm{e} \\
3 & \mathrm{P} \mathrm{e} \\
3 & \mathrm{D} \mathrm{e} \\
3 & \mathrm{SPD} \text { e } \\
3 & \mathrm{PD} \mathrm{e}\end{array}$ \\
\hline $\begin{array}{l}N l v=3, \quad 1, \mathrm{e}: \\
2 \mathrm{~s} 22 \mathrm{p} \\
2 \mathrm{~s} 22 \mathrm{p} \\
2 \mathrm{~s} 22 \mathrm{p} \\
N l v(\mathrm{c})=3 \text { : set }\end{array}$ & $\begin{array}{l}\mathrm{S}(0) \mathrm{P} \\
(2 \mathrm{Po}) \\
\text { (2Po) } \\
(2 \mathrm{Po}) \\
\text { complete }\end{array}$ & $\begin{array}{l}(1) \mathrm{I} \\
3 / 2 \\
3 / 2 \\
3 / 2\end{array}$ & $\begin{array}{l}(2) \\
3 \mathrm{p} \\
3 \mathrm{p} \\
3 \mathrm{p}\end{array}$ & $\begin{array}{l}1 \\
2 \\
0\end{array}$ & $\begin{array}{l}-5.15048 \mathrm{E}+01 \\
-5.07154 \mathrm{E}+01 \\
-4.99106 \mathrm{E}+01\end{array}$ & $\begin{array}{l}2.90 \\
2.92 \\
2.94\end{array}$ & $\begin{array}{ll}1 & \mathrm{P} \\
1 & \mathrm{e} \\
1 & \mathrm{D} \mathrm{e} \\
1 & \mathrm{~S} \mathrm{e}\end{array}$ \\
\hline $\begin{array}{l}N l v=3, \quad 5, \mathrm{e}: \\
2 \mathrm{~s} 2 \mathrm{p} 2 \\
2 \mathrm{~s} 2 \mathrm{p} 2 \\
2 \mathrm{~s} 2 \mathrm{p} 2 \\
N l v(c)=3 \text { : set }\end{array}$ & 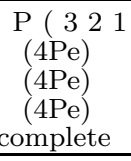 & $\begin{array}{l}\text { ) } \\
1 / 2 \\
3 / 2 \\
5 / 2\end{array}$ & $\begin{array}{l}3 \mathrm{~s} \\
3 \mathrm{~s} \\
3 \mathrm{~s}\end{array}$ & $\begin{array}{l}1 \\
2 \\
3\end{array}$ & $\begin{array}{l}-5.09584 \mathrm{E}+01 \\
-5.05218 \mathrm{E}+01 \\
-5.00672 \mathrm{E}+01 \\
\end{array}$ & $\begin{array}{l}2.84 \\
2.84 \\
2.84\end{array}$ & $\begin{array}{lll}5 & \mathrm{P} & \mathrm{e} \\
5 & \mathrm{P} & \mathrm{e} \\
5 & \mathrm{P} & \mathrm{e}\end{array}$ \\
\hline $\begin{array}{l}N l v=3, \quad 1, \mathrm{o}: \\
2 \mathrm{~s} 22 \mathrm{p} \\
2 \mathrm{~s} 22 \mathrm{p} \\
2 \mathrm{~s} 22 \mathrm{p} \\
N l v(\mathrm{c})=3: \text { set }\end{array}$ & $\begin{array}{l}\mathrm{P}(1) \mathrm{D} \\
(2 \mathrm{Po}) \\
(2 \mathrm{Po}) \\
\text { (2Po) } \\
\text { complete }\end{array}$ & $\begin{array}{l}(2) \\
1 / 2 \\
3 / 2 \\
3 / 2\end{array}$ & $\begin{array}{l}\mathrm{F}(3) \\
3 \mathrm{~d} \\
3 \mathrm{~d} \\
3 \mathrm{~d}\end{array}$ & $\begin{array}{l}2 \\
3 \\
1\end{array}$ & $\begin{array}{l}-5.05889 \mathrm{E}+01 \\
-4.85779 \mathrm{E}+01 \\
-4.84796 \mathrm{E}+01 \\
\end{array}$ & $\begin{array}{l}2.95 \\
2.98 \\
2.98\end{array}$ & $\begin{array}{lll}1 & \mathrm{D} & \mathrm{o} \\
1 & \mathrm{~F} & \mathrm{o} \\
1 & \mathrm{P} & \mathrm{o}\end{array}$ \\
\hline $\begin{array}{l}N l v=3, \quad 3, \mathrm{e}: \\
2 \mathrm{~s} 2 \mathrm{p} 2 \\
2 \mathrm{~s} 2 \mathrm{p} 2 \\
2 \mathrm{~s} 2 \mathrm{p} 2 \\
N l v(\mathrm{c})=3: \text { set }\end{array}$ & $\begin{array}{c}\mathrm{P}(2)^{2} 10 \\
(4 \mathrm{Pe}) \\
(4 \mathrm{Pe}) \\
(4 \mathrm{Pe}) \\
\text { complete }\end{array}$ & $\begin{array}{l}) \\
1 / 2 \\
3 / 2 \\
5 / 2\end{array}$ & $\begin{array}{l}3 \mathrm{~s} \\
3 \mathrm{~s} \\
3 \mathrm{~s}\end{array}$ & $\begin{array}{l}0 \\
1 \\
2\end{array}$ & $\begin{array}{l}-5.03119 \mathrm{E}+01 \\
-4.96425 \mathrm{E}+01 \\
-4.91481 \mathrm{E}+01 \\
\end{array}$ & $\begin{array}{l}2.86 \\
2.86 \\
2.86\end{array}$ & 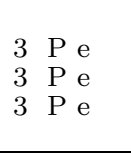 \\
\hline $\begin{array}{l}N l v=9, \quad 3, \mathrm{o}: \\
2 \mathrm{~s} 22 \mathrm{p} \\
2 \mathrm{~s} 22 \mathrm{p} \\
2 \mathrm{~s} 22 \mathrm{p} \\
2 \mathrm{~s} 22 \mathrm{p} \\
2 \mathrm{~s} 22 \mathrm{p} \\
2 \mathrm{~s} 22 \mathrm{p} \\
2 \mathrm{~s} 22 \mathrm{p} \\
2 \mathrm{~s} 22 \mathrm{p} \\
2 \mathrm{~s} 22 \mathrm{p} \\
N l v(\mathrm{c})=9: \text { set }\end{array}$ & $\begin{array}{l}\mathrm{P}(210 \\
(2 \mathrm{Po}) \\
(2 \mathrm{Po}) \\
(2 \mathrm{Po}) \\
(2 \mathrm{Po}) \\
(2 \mathrm{Po}) \\
(2 \mathrm{Po}) \\
(2 \mathrm{Po}) \\
\text { (2Po) } \\
\text { (2Po) } \\
\text { complete } \\
\end{array}$ & $\begin{array}{l}\text { D }( \\
1 / 2 \\
1 / 2 \\
1 / 2 \\
3 / 2 \\
3 / 2 \\
3 / 2 \\
3 / 2 \\
3 / 2 \\
3 / 2\end{array}$ & $\begin{array}{l}321 \\
3 d \\
3 d \\
3 d \\
3 d \\
3 d \\
3 d \\
3 d \\
3 d \\
3 d\end{array}$ & $\begin{array}{l}\mathrm{F} \\
3 \\
2 \\
1 \\
2 \\
4 \\
3 \\
1 \\
2 \\
0\end{array}$ & $\begin{array}{l}432 . \\
-5.00116 \mathrm{E}+01 \\
-4.99604 \mathrm{E}+01 \\
-4.98528 \mathrm{E}+01 \\
-4.94634 \mathrm{E}+01 \\
-4.94577 \mathrm{E}+01 \\
-4.94017 \mathrm{E}+01 \\
-4.92676 \mathrm{E}+01 \\
-4.92368 \mathrm{E}+01 \\
-4.90612 \mathrm{E}+01\end{array}$ & $\begin{array}{l}2.97 \\
2.97 \\
2.97 \\
2.95 \\
2.95 \\
2.96 \\
2.96 \\
2.96 \\
2.97\end{array}$ & $\begin{array}{ll}3 & \mathrm{DF} \text { o } \\
3 & \mathrm{PDF} \text { o } \\
3 & \mathrm{PD} \mathrm{o} \\
3 & \mathrm{PDF} \text { o } \\
3 & \mathrm{~F} \mathrm{o} \\
3 & \mathrm{DF} \text { o } \\
3 & \mathrm{PD} \mathrm{o} \\
3 & \mathrm{PDF} \text { o } \\
3 & \mathrm{P} \mathrm{o}\end{array}$ \\
\hline $\begin{array}{l}N l v=9, \quad 5, \mathrm{o}: \\
2 \mathrm{~s} 2 \mathrm{p} 2 \\
2 \mathrm{~s} 2 \mathrm{p} 2 \\
2 \mathrm{~s} 2 \mathrm{p} 2 \\
2 \mathrm{~s} 2 \mathrm{p} 2 \\
2 \mathrm{~s} 2 \mathrm{p} 2 \\
2 \mathrm{~s} 2 \mathrm{p} 2 \\
2 \mathrm{~s} 2 \mathrm{p} 2 \\
2 \mathrm{~s} 2 \mathrm{p} 2 \\
2 \mathrm{~s} 2 \mathrm{p} 2 \\
N l v(\mathrm{c})=9: \text { set }\end{array}$ & $\begin{array}{c}\mathrm{S}(22) \mathrm{P} \\
(4 \mathrm{Pe}) \\
(4 \mathrm{Pe}) \\
(4 \mathrm{Pe}) \\
(4 \mathrm{Pe}) \\
(4 \mathrm{Pe}) \\
(4 \mathrm{Pe}) \\
(4 \mathrm{Pe}) \\
(4 \mathrm{Pe}) \\
(4 \mathrm{Pe}) \\
\text { complete } \\
\end{array}$ & $\begin{array}{l}\text { ( } 32 \\
1 / 2 \\
1 / 2 \\
1 / 2 \\
1 / 2 \\
3 / 2 \\
3 / 2 \\
5 / 2 \\
5 / 2 \\
5 / 2\end{array}$ & $\begin{array}{l}) \mathrm{D} \\
3 \mathrm{p} \\
3 \mathrm{p} \\
3 \mathrm{p} \\
3 \mathrm{p} \\
3 \mathrm{p} \\
3 \mathrm{p} \\
3 \mathrm{p} \\
3 \mathrm{p} \\
3 \mathrm{p}\end{array}$ & $\begin{array}{l}43 \\
1 \\
0 \\
2 \\
1 \\
3 \\
2 \\
4 \\
3 \\
2\end{array}$ & $\begin{array}{l}210) \\
-4.89841 \mathrm{E}+01 \\
-4.89298 \mathrm{E}+01 \\
-4.88377 \mathrm{E}+01 \\
-4.87720 \mathrm{E}+01 \\
-4.85537 \mathrm{E}+01 \\
-4.82586 \mathrm{E}+01 \\
-4.77166 \mathrm{E}+01 \\
-4.76095 \mathrm{E}+01 \\
-4.72496 \mathrm{E}+01\end{array}$ & $\begin{array}{l}2.89 \\
2.89 \\
2.90 \\
2.90 \\
2.89 \\
2.90 \\
2.90 \\
2.90 \\
2.91\end{array}$ & 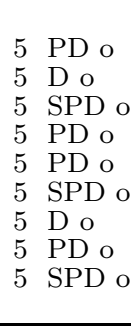 \\
\hline $\begin{array}{l}N l v=3, \quad 3, \mathrm{e}: \\
2 \mathrm{~s} 2 \mathrm{p} 2 \\
2 \mathrm{~s} 2 \mathrm{p} 2 \\
2 \mathrm{~s} 2 \mathrm{p} 2 \\
N l v(\mathrm{c})=3 \text { : set }\end{array}$ & 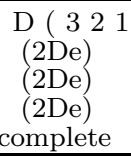 & $\begin{array}{l}\text { ) } \\
3 / 2 \\
5 / 2 \\
5 / 2\end{array}$ & $\begin{array}{l}3 \mathrm{~s} \\
3 \mathrm{~s} \\
3 \mathrm{~s}\end{array}$ & $\begin{array}{l}1 \\
2 \\
3\end{array}$ & $\begin{array}{l}-4.78489 \mathrm{E}+01 \\
-4.77709 \mathrm{E}+01 \\
-4.76659 \mathrm{E}+01\end{array}$ & $\begin{array}{l}2.84 \\
2.84 \\
2.84\end{array}$ & $\begin{array}{ll}3 & \mathrm{D} e \\
3 & \mathrm{D} \\
3 & \mathrm{D} \\
\mathrm{e}\end{array}$ \\
\hline
\end{tabular}


same configuration to show the correspondence between two sets of representations, in $J$-levels and in $L S$ terms. (Listing of the lowest levels of equivalent electron states are omitted as they are given in energy comparison table). The grouping of levels provides the check for completeness of sets of energy levels that should belong to the corresponding $L S$ term, and detects any missing level. The title line of each set of levels in the tables lists spin multiplicity $(2 S+1)$, parity, all possible $L$-values that can be formed from the core or target term, and outer or the valence electron angular momentum. The $J$-values for each possible $L S$ term are specified within the parentheses next to the value of $L$. "Nlv" is the total number of $J$-levels that are expected from this set of $L S$ terms. This line is followed by the set of BPRM energy levels of same configurations. "Nlv(c)", at the end of the set, specifies the total number of $J$-levels obtained. If $N l v=N l v(\mathrm{c})$ for a set, the calculated energy set is complete. The correspondence of couplings and completeness of levels are carried out by the program PRCBPID which also detects and prints the missing levels. For example, in the set for ${ }^{3}(\mathrm{G}, \mathrm{H}, \mathrm{I})^{\mathrm{e}}$ for Ar XIII near the end of Table 2b, the set is found to be incomplete where four levels of $J=5,4,6,5$ are missing. Sets with missing levels usually lie in the high energy region. Each level of a set is further identified by all possible $L S$ terms (specified in the last column of the set). The multiple $L S$ terms can be reduced to the most probable (but approximately) one using Hund's rule, as explained above. It may be noted that levels are grouped consistently in closely spaced energies and in effective quantum numbers confirming proper designation of the $L S$ terms.

The BPRM energy levels for Ar XIII and Fe XXI are compared in Table 4 with the limited number of levels observed. The 55 observed levels of Ar XIII (Kelly, NIST) are not in the compiled list by the NIST. However, the calculated fine structure energies agree with these observed ones to about $1 \%$ for most of the levels. The difference is upto $9 \%$. The agreement between the observed and calculated energies is much better for Fe XXI (Table 4). There are 39 observed energy levels of Fe XXI (Sugar \& Corliss 1985). Present energies agree with the observed ones to less than or about $1 \%$ for all except two levels that agree within $2 \%$. In Table 4 the column next to the $J$-column is for the energy level index, $I_{J}$. The energy level index specifies the position, in ascending order, of the level in the calculated set of energies of $J \pi$ symmetry. It is necessary to use the level indices to make correspondence between the calculated and observed levels for later use.

\subsection{Oscillators strengths}

The total number of oscillator strengths ( $f$-values) for bound-bound fine structure transitions is 198259 for Ar XIII and is 300079 for Fe XXI. These include both the dipole allowed $(\Delta S=0)$ and the intercombination
$(\Delta S \neq 0)$ transitions. Complete sets of $f$-values for both Ar XIII and Fe XXI are available electronically.

Partial sets of the oscillator strengths for Ar XIII and Fe XXI are presented in Tables 5 and 6 respectively. The format of the tables is the same as that of the electronic files for the $f$-values. At the top of each table, the two numbers are the nuclear charge $(Z=18$ for Ar XIII and $=26$ for Fe XXI) and number of electrons in the ion, $N_{\text {elc }}$ (=6 for carbon like ions). Below this line are the sets of oscillator strengths belonging to a pair of symmetries, $J_{i} \pi_{i}-J_{k} \pi_{k}$, specified at the top. The symmetries are expressed in the form of $2 J_{i}$ and $\pi_{i}(\pi=0$ for even and $=1$ for odd parity), $2 J_{k}$ and $\pi_{k}$. For example, Tables 5 and 6 present partial transitions among the levels of symmetries $J=0^{\mathrm{e}}$ and $J=1^{\circ}$ for Ar XIII and Fe XXI respectively. The line following the transition symmetries are the number of bound levels, $N_{J i}$ and $N_{J k}$. This line is followed by $N_{J i} \times N_{J k}$ number of transitions. The first two columns are the energy level indices, $I_{i}$ and $I_{k}$ (as mentioned above), and the third and the fourth columns are their energies, $E_{i}$ and $E_{k}$, in Rydberg unit. The fifth column is the $g f_{\mathrm{L}}$ for the allowed transitions $(\Delta J=0, \pm 1) . f_{\mathrm{L}}$ is the oscillator strength in length form, and $g=2 J+1$ is the statistical weight factor of the initial or the lower level. A negative value for $g f$ means that $i$ is the lower level, while a positive one means that $k$ is the lower level. Column six is the line strength $(S)$. The last column in the table gives the transition probability, $A_{k i}\left(\mathrm{~s}^{-1}\right)$. Complete spectroscopic identification of the transition can be obtained from Tables $2 \mathrm{a}$ and $3 \mathrm{a}$ by referring to the values of $J_{i} \pi_{i}, I_{i}, J_{k} \pi_{k}$, and $I_{k}$. For example, the first transition for Fe XXI in Table 6 corresponds to, as identified in Table 3a, dipole allowed transition $2 \mathrm{~s}^{2} 2 \mathrm{p}^{2}\left({ }^{3} \mathrm{P}_{0}{ }_{0}\right)\left(I_{i}=1\right) \rightarrow$ $2 \mathrm{~s} 2 \mathrm{p}^{3}\left({ }^{3} \mathrm{D}^{\mathrm{o}}{ }_{1}\right)\left(I_{k}=1\right)$.

A set of transition probabilities for both Ar XIII and Fe XXI has been reprocessed such that observed energy differences, rather than the calculated ones, are used to obtain the $f$ - and $A$-values from BPRM line strengths $(S)$. The $S$-values are energy independent quantities. As the observed energies have lower uncertainties than the calculated ones, use of them, instead of the calculated energies, with the $S$-values (Eqs. 7 and 8) improves the accuracy of the $f$ - and $A$-values for the relevant transitions. (This is a commonly used procedure adopted first in the NIST compilation.) The astrophysical models also in general use the observed transition energies for the relevant $f$-, $S$ and $A$-values. For any comparison or spectral diagnostics, therefore, values from these sets should be used.

The reprocessing of $f$ - and $A$-values has been carried out for all the allowed transitions $(\Delta J=0, \pm 1)$ among the observed levels. The set consists of 333 transitions of Ar XII and 184 of Fe XXI (these sets are also available electronically). Sample sets of the reprocessed oscillator strengths are presented in Tables 7a and 8a for Ar XIII and Fe XXI. Transitions are listed in $J \pi$ order, and in 
Table 4. Comparison of absolute calculated BPRM energies $\left(E_{\mathrm{c}}\right)$ with the observed ones $\left(E_{\mathrm{o}}\right.$, Sugar \& Corliss 1985$)$ for Ar XIII and Fe XXI. $I_{J}$ is the level index for the energy position in symmetry $J \pi$. The asterisk next to the $J$-value indicates that the term has incomplete set of observed fine structure levels

\begin{tabular}{|c|c|c|c|c|c|c|c|c|c|c|c|}
\hline \multicolumn{2}{|c|}{ Level } & $J$ & $\bar{I} I_{J}$ & $E_{\mathrm{o}}(\mathrm{Ry})$ & $E_{\mathrm{c}}(\mathrm{Ry})$ & Level & & $J$ & $\overline{I J}$ & $E_{\mathrm{o}}(\mathrm{Ry})$ & $E_{\mathrm{c}}(\mathrm{Ry})$ \\
\hline \multicolumn{12}{|c|}{ Ar XIII } \\
\hline $2 \mathrm{~s}^{2} 2 \mathrm{p}^{2}$ & ${ }^{3} \mathrm{P}$ & 2 & 1 & -50.133 & -50.209 & $2 \mathrm{~s}^{2} 2 \mathrm{p} 3 \mathrm{~d}$ & ${ }^{3} \mathrm{P}^{\mathrm{o}}$ & 2 & 9 & -19.053 & -19.149 \\
\hline $2 \mathrm{~s}^{2} 2 \mathrm{p}^{2}$ & ${ }^{3} \mathrm{P}$ & 1 & 1 & -50.243 & -50.326 & $2 \mathrm{~s}^{2} 2 \mathrm{p} 3 \mathrm{~d}$ & ${ }^{3} \mathrm{P}^{\mathrm{o}}$ & 1 & 8 & -19.037 & -19.112 \\
\hline $2 \mathrm{~s}^{2} 2 \mathrm{p}^{2}$ & ${ }^{3} \mathrm{P}$ & 0 & 1 & -50.332 & -50.416 & $2 \mathrm{~s}^{2} 2 \mathrm{p} 3 \mathrm{~d}$ & ${ }^{3} \mathrm{P}^{\mathrm{o}}$ & 0 & 3 & -19.025 & -19.079 \\
\hline $2 \mathrm{~s}^{2} 2 \mathrm{p}^{2}$ & ${ }^{1} \mathrm{D}$ & 2 & 2 & -49.558 & -49.621 & $2 \mathrm{~s}^{2} 2 \mathrm{p} 3 \mathrm{~d}$ & ${ }^{1} \mathrm{P}^{\mathrm{o}}$ & 1 & 9 & -18.740 & -18.772 \\
\hline $2 \mathrm{~s}^{2} 2 \mathrm{p}^{2}$ & ${ }^{1} \mathrm{~S}$ & 0 & 2 & -48.856 & -48.905 & $2 \mathrm{~s}^{2} 2 \mathrm{p} 3 \mathrm{~d}$ & ${ }^{1} \mathrm{~F}^{\mathrm{o}}$ & 3 & 4 & -18.736 & -18.787 \\
\hline $2 \mathrm{~s} 2 \mathrm{p}^{3}$ & ${ }^{5} \mathrm{~S}^{\circ}$ & 2 & 1 & -48.274 & -48.346 & $2 \mathrm{~s} 2 \mathrm{p}^{2} 3 \mathrm{p}$ & ${ }^{3} \mathrm{D}^{\circ}$ & 3 & 8 & -17.126 & -16.822 \\
\hline $2 \mathrm{~s} 2 \mathrm{p}^{3}$ & ${ }^{3} \mathrm{D}^{\circ}$ & 3 & 1 & -46.469 & -46.508 & $2 \mathrm{~s} 2 \mathrm{p}^{2} 3 \mathrm{p}$ & ${ }^{3} \mathrm{D}^{\mathrm{o}}$ & 2 & 16 & -17.126 & -16.805 \\
\hline $2 \mathrm{~s} 2 \mathrm{p}^{3}$ & ${ }^{3} \mathrm{D}^{\circ}$ & 2 & 2 & -46.480 & -46.533 & $2 \mathrm{~s} 2 \mathrm{p}^{2} 3 \mathrm{p}$ & ${ }^{3} \mathrm{D}^{\circ}$ & 1 & 16 & -17.126 & -16.605 \\
\hline $2 \mathrm{~s} 2 \mathrm{p}^{3}$ & ${ }^{3} \mathrm{D}^{\circ}$ & 1 & 1 & -46.476 & -46.527 & $2 \mathrm{~s} 2 \mathrm{p}^{2}\left({ }^{4} \mathrm{P}\right) 3 \mathrm{~d}$ & ${ }^{5} \mathrm{P}$ & $3^{*}$ & 6 & -17.117 & -17.281 \\
\hline $2 \mathrm{~s} 2 \mathrm{p}^{3}$ & ${ }^{3} \mathrm{P}^{\mathrm{o}}$ & 2 & 3 & -45.803 & -45.826 & $2 \mathrm{~s} 2 \mathrm{p}^{2}\left({ }^{4} \mathrm{P}\right) 3 \mathrm{~d}$ & ${ }^{5} \mathrm{P}$ & $2^{*}$ & 14 & -17.074 & -17.274 \\
\hline $2 \mathrm{~s} 2 \mathrm{p}^{3}$ & ${ }^{3} \mathrm{P}^{\mathrm{o}}$ & 1 & 2 & -45.814 & -45.844 & $2 \mathrm{~s} 2 \mathrm{p}^{2} 3 \mathrm{p}$ & ${ }^{1} \mathrm{D}^{\mathrm{o}}$ & 2 & 15 & -16.019 & -16.867 \\
\hline $2 \mathrm{~s} 2 \mathrm{p}^{3}$ & ${ }^{3} \mathrm{P}^{\mathrm{o}}$ & 0 & 1 & -45.817 & -45.852 & $2 \mathrm{~s} 2 \mathrm{p}^{2} 3 \mathrm{p}$ & ${ }^{3} \mathrm{P}^{\mathrm{o}}$ & 2 & 19 & -15.784 & -15.731 \\
\hline $2 \mathrm{~s} 2 \mathrm{p}^{3}$ & ${ }^{1} \mathrm{D}^{\circ}$ & 2 & 4 & -44.630 & -44.627 & $2 \mathrm{~s}^{2} \mathrm{p}^{2} 3 \mathrm{p}$ & ${ }^{3} \mathrm{P}^{\mathrm{o}}$ & 1 & 18 & -15.784 & -15.798 \\
\hline $2 \mathrm{~s} 2 \mathrm{p}^{3}$ & ${ }^{3} \mathrm{~S}^{\circ}$ & 1 & 3 & -44.604 & -44.597 & $2 \mathrm{~s} 2 \mathrm{p}^{2} 3 \mathrm{p}$ & ${ }^{3} \mathrm{P}^{\mathrm{o}}$ & 0 & 8 & -15.784 & -15.721 \\
\hline $2 \mathrm{~s} 2 \mathrm{p}^{3}$ & ${ }^{1} \mathrm{P}^{\mathrm{o}}$ & 1 & 4 & -43.966 & -43.949 & $2 \mathrm{~s} 2 \mathrm{p}^{2} 3 \mathrm{p}$ & ${ }^{1} \mathrm{P}^{\mathrm{o}}$ & 1 & 20 & -15.581 & -15.589 \\
\hline $2 \mathrm{~s}^{2} 2 \mathrm{p} 3 \mathrm{~s}$ & ${ }^{3} \mathrm{P}^{\mathrm{o}}$ & 2 & 5 & -21.535 & -21.845 & $2 \mathrm{~s} 2 \mathrm{p}^{2} 3 \mathrm{p}$ & ${ }^{3} \mathrm{~S}^{\mathrm{o}}$ & 1 & 21 & -15.563 & -15.475 \\
\hline $2 \mathrm{~s}^{2} 2 \mathrm{p} 3 \mathrm{~s}$ & ${ }^{3} \mathrm{P}^{\mathrm{o}}$ & 1 & 5 & -21.659 & -22.001 & $2 \mathrm{~s}^{2} 2 \mathrm{p} 4 \mathrm{~s}$ & ${ }^{1} \mathrm{P}^{\mathrm{o}}$ & 1 & 31 & -11.826 & -12.379 \\
\hline $2 \mathrm{~s}^{2} 2 \mathrm{p} 3 \mathrm{~s}$ & ${ }^{3} \mathrm{P}^{\circ}$ & 0 & 2 & -21.722 & -22.058 & $2 \mathrm{~s}^{2} 2 \mathrm{p} 4 \mathrm{~s}$ & ${ }^{3} \mathrm{P}^{\circ}$ & 2 & 29 & -11.580 & -12.395 \\
\hline $2 \mathrm{~s}^{2} 2 \mathrm{p} 3 \mathrm{~s}$ & ${ }^{1} \mathrm{P}^{\mathrm{o}}$ & 1 & 6 & -21.381 & -21.725 & $2 \mathrm{~s}^{2} 2 \mathrm{p} 4 \mathrm{~s}$ & ${ }^{3} \mathrm{P}^{\mathrm{o}}$ & 1 & 30 & -11.580 & -12.588 \\
\hline $2 \mathrm{~s}^{2} 2 \mathrm{p} 3 \mathrm{~d}$ & ${ }^{3} \mathrm{~F}^{\circ}$ & 4 & 1 & -19.286 & -19.358 & $2 \mathrm{~s}^{2} 2 \mathrm{p} 4 \mathrm{~s}$ & ${ }^{3} \mathrm{P}^{\mathrm{o}}$ & 0 & 12 & -11.580 & -12.602 \\
\hline $2 \mathrm{~s}^{2} 2 \mathrm{p} 3 \mathrm{~d}$ & ${ }^{3} \mathrm{~F}^{\mathrm{o}}$ & 3 & 2 & -19.419 & -19.463 & $2 \mathrm{~s}^{2} 2 \mathrm{p} 4 \mathrm{~d}$ & ${ }^{1} \mathrm{P}^{\mathrm{o}}$ & 1 & 45 & -10.774 & -10.520 \\
\hline $2 \mathrm{~s}^{2} 2 \mathrm{p} 3 \mathrm{~d}$ & ${ }^{3} \mathrm{~F}^{\mathrm{o}}$ & 2 & 7 & -19.520 & -19.428 & $2 \mathrm{~s}^{2} 2 \mathrm{p} 4 \mathrm{~d}$ & ${ }^{1} \mathrm{D}^{\mathrm{o}}$ & 2 & 46 & -10.599 & -10.641 \\
\hline $2 \mathrm{~s}^{2} 2 \mathrm{p} 3 \mathrm{~d}$ & ${ }^{1} \mathrm{D}^{\mathrm{o}}$ & 2 & 6 & -19.398 & -19.575 & $2 \mathrm{~s}^{2} 2 \mathrm{p} 4 \mathrm{~d}$ & ${ }^{3} \mathrm{P}^{\mathrm{o}}$ & 2 & 44 & -10.565 & -10.835 \\
\hline $2 \mathrm{~s}^{2} 2 \mathrm{p} 3 \mathrm{~d}$ & ${ }^{3} \mathrm{D}^{\circ}$ & 3 & 3 & -19.106 & -19.177 & $2 \mathrm{~s}^{2} 2 \mathrm{p} 4 \mathrm{~d}$ & ${ }^{3} \mathrm{P}^{\mathrm{o}}$ & 1 & 43 & -10.565 & -10.785 \\
\hline $2 \mathrm{~s}^{2} 2 \mathrm{p} 3 \mathrm{~d}$ & ${ }^{3} \mathrm{D}^{\circ}$ & 2 & 8 & -19.194 & -19.208 & $2 \mathrm{~s}^{2} 2 \mathrm{p} 4 \mathrm{~d}$ & ${ }^{3} \mathrm{P}^{\mathrm{o}}$ & 0 & 18 & -10.565 & -10.625 \\
\hline $2 \mathrm{~s}^{2} 2 \mathrm{p} 3 \mathrm{~d}$ & ${ }^{3} \mathrm{D}^{\circ}$ & 1 & 7 & -19.253 & -19.258 & & & & & & \\
\hline \multicolumn{12}{|c|}{ Fe XXI } \\
\hline $2 \mathrm{~s}^{2} 2 \mathrm{p}^{2}$ & ${ }^{3} \mathrm{P}$ & 2 & 1 & -123.050 & -123.185 & $2 \mathrm{~s}^{2} 2 \mathrm{p} 3 \mathrm{~d}$ & ${ }^{1} \mathrm{D}^{\mathrm{o}}$ & 2 & 6 & -50.320 & -50.589 \\
\hline $2 \mathrm{~s}^{2} 2 \mathrm{p}^{2}$ & ${ }^{3} \mathrm{P}$ & 1 & 1 & -123.440 & -123.615 & $2 \mathrm{~s}^{2} 2 \mathrm{p} 3 \mathrm{~d}$ & ${ }^{3} \mathrm{~F}^{\mathrm{o}}$ & $3^{*}$ & 2 & -50.289 & -50.012 \\
\hline $2 \mathrm{~s}^{2} 2 \mathrm{p}^{2}$ & ${ }^{3} \mathrm{P}$ & 0 & 1 & -124.110 & -124.292 & $2 \mathrm{~s}^{2} 2 \mathrm{p} 3 \mathrm{~d}$ & ${ }^{3} \mathrm{D}^{\mathrm{o}}$ & $3^{*}$ & 3 & -49.436 & -49.402 \\
\hline $2 \mathrm{~s}^{2} 2 \mathrm{p}^{2}$ & ${ }^{1} \mathrm{D}$ & 2 & 2 & -121.890 & -122.009 & $2 \mathrm{~s}^{2} 2 \mathrm{p} 3 \mathrm{~d}$ & ${ }^{3} \mathrm{D}^{\mathrm{o}}$ & $2^{*}$ & 8 & -49.506 & -49.463 \\
\hline $2 \mathrm{~s}^{2} 2 \mathrm{p}^{2}$ & ${ }^{1} \mathrm{~S}$ & 0 & 2 & -120.730 & -120.849 & $2 \mathrm{~s}^{2} 2 \mathrm{p} 3 \mathrm{~d}$ & ${ }^{3} \mathrm{P}^{\mathrm{o}}$ & $2^{*}$ & 9 & -49.109 & -49.237 \\
\hline $2 \mathrm{~s} 2 \mathrm{p}^{3}$ & ${ }^{5} \mathrm{~S}^{\mathrm{O}}$ & 2 & 1 & -119.680 & -119.850 & $2 \mathrm{~s}^{2} 2 \mathrm{p} 3 \mathrm{~d}$ & ${ }^{1} \mathrm{P}^{\mathrm{o}}$ & 1 & 11 & -48.535 & -48.480 \\
\hline $2 \mathrm{~s} 2 \mathrm{p}^{3}$ & ${ }^{3} \mathrm{D}^{\circ}$ & 3 & 1 & -116.790 & -116.897 & $2 \mathrm{~s}^{2} 2 \mathrm{p} 3 \mathrm{~d}$ & ${ }^{1} \mathrm{~F}^{\mathrm{o}}$ & 3 & 4 & -48.355 & -48.578 \\
\hline $2 \mathrm{~s} 2 \mathrm{p}^{3}$ & ${ }^{3} \mathrm{D}^{\circ}$ & 2 & 2 & -117.030 & -117.155 & $2 \mathrm{~s}^{2} 2 \mathrm{p} 4 \mathrm{~d}$ & ${ }^{3} \mathrm{~F}^{\mathrm{o}}$ & $3^{*}$ & 22 & -27.939 & -27.934 \\
\hline $2 \mathrm{~s} 2 \mathrm{p}^{3}$ & ${ }^{3} \mathrm{D}^{\circ}$ & 1 & 1 & -117.040 & -117.168 & $2 \mathrm{~s}^{2} 2 \mathrm{p} 4 \mathrm{~d}$ & ${ }^{3} \mathrm{P}^{\mathrm{o}}$ & $2^{*}$ & 39 & -27.703 & -27.949 \\
\hline $2 \mathrm{~s} 2 \mathrm{p}^{3}$ & ${ }^{3} \mathrm{P}^{\mathrm{o}}$ & 2 & 3 & -115.530 & -115.651 & $2 \mathrm{~s}^{2} 2 \mathrm{p} 4 \mathrm{~d}$ & ${ }^{3} \mathrm{P}^{\mathrm{o}}$ & $1^{*}$ & 40 & -26.718 & -26.990 \\
\hline $2 \mathrm{~s} 2 \mathrm{p}^{3}$ & ${ }^{3} \mathrm{P}^{\mathrm{o}}$ & 1 & 2 & -115.690 & -115.815 & $2 \mathrm{~s}^{2} 2 \mathrm{p} 4 \mathrm{~d}$ & ${ }^{3} \mathrm{D}^{\mathrm{o}}$ & 3 & 23 & -26.718 & -27.064 \\
\hline $2 \mathrm{~s} 2 \mathrm{p}^{3}$ & ${ }^{3} \mathrm{P}^{\mathrm{o}}$ & 0 & 1 & -115.760 & -115.895 & $2 \mathrm{~s}^{2} 2 \mathrm{p} 4 \mathrm{~d}$ & ${ }^{3} \mathrm{D}^{\circ}$ & 2 & 41 & -27.019 & -27.005 \\
\hline $2 \mathrm{~s} 2 \mathrm{p}^{3}$ & ${ }^{3} \mathrm{~S}^{\mathrm{o}}$ & 1 & 3 & -114.130 & -114.241 & $2 \mathrm{~s}^{2} 2 \mathrm{p} 4 \mathrm{~d}$ & ${ }^{3} \mathrm{D}^{\mathrm{o}}$ & 1 & 39 & -27.693 & -27.887 \\
\hline $2 \mathrm{~s} 2 \mathrm{p}^{3}$ & ${ }^{1} \mathrm{D}^{\mathrm{o}}$ & 2 & 4 & -113.850 & -113.922 & $2 \mathrm{~s}^{2} 2 \mathrm{p} 4 \mathrm{~d}$ & ${ }^{1} \mathrm{D}^{\mathrm{o}}$ & 2 & 40 & -26.837 & -27.069 \\
\hline $2 \mathrm{~s} 2 \mathrm{p}^{3}$ & ${ }^{1} \mathrm{P}^{\mathrm{o}}$ & 1 & 4 & -112.620 & -112.695 & $2 \mathrm{~s}^{2} 2 \mathrm{p} 4 \mathrm{~d}$ & ${ }^{1} \mathrm{~F}^{\mathrm{o}}$ & 3 & 24 & -26.782 & -26.785 \\
\hline $2 p^{4}$ & ${ }^{3} \mathrm{P}$ & 2 & 3 & -109.110 & -109.226 & $2 \mathrm{~s}^{2} 2 \mathrm{p} 5 \mathrm{~d}$ & ${ }^{3} \mathrm{D}^{\mathrm{o}}$ & $3^{*}$ & 50 & -16.567 & -16.849 \\
\hline $2 p^{4}$ & ${ }^{3} \mathrm{P}$ & 1 & 2 & -108.250 & -108.362 & $2 \mathrm{~s}^{2} 2 \mathrm{p} 5 \mathrm{~d}$ & ${ }^{3} \mathrm{P}^{\mathrm{o}}$ & $1^{*}$ & 67 & -16.494 & -16.813 \\
\hline $2 p^{4}$ & ${ }^{3} \mathrm{P}$ & 0 & 3 & -108.300 & -108.406 & $2 \mathrm{~s}^{2} 2 \mathrm{p} 5 \mathrm{~d}$ & ${ }^{1} \mathrm{D}^{\mathrm{o}}$ & 2 & 72 & -16.494 & -16.854 \\
\hline $2 p^{4}$ & ${ }^{1} \mathrm{D}$ & 2 & 4 & -107.550 & -107.626 & $2 \mathrm{~s}^{2} 2 \mathrm{p} 5 \mathrm{~d}$ & ${ }^{1} \mathrm{~F}^{\mathrm{o}}$ & 3 & 51 & -16.457 & -16.724 \\
\hline $2 p^{4}$ & ${ }^{1} \mathrm{~S}$ & 0 & 4 & -105.450 & -105.511 & & & & & & \\
\hline
\end{tabular}


Table 5. Oscillator strengths and transition probabilities for Ar XIII (see text for explanation)

\begin{tabular}{|c|c|c|c|c|c|c|}
\hline \multicolumn{7}{|c|}{$\begin{array}{ll}18 & 6\end{array}$} \\
\hline & 0 & 2 & & & & \\
\hline 56 & 136 & $E_{i}(\mathrm{Ry})$ & $E_{j}(\mathrm{Ry})$ & $g f_{\mathrm{L}}$ & $S$ & $A_{j i}\left(\mathrm{~s}^{-1}\right)$ \\
\hline 1 & 1 & $-5.04159 \mathrm{E}+01$ & $-4.65272 \mathrm{E}+01$ & $-7.432 \mathrm{E}-02$ & $5.734 \mathrm{E}-02$ & $3.009 \mathrm{E}+09$ \\
\hline 1 & 2 & $-5.04159 \mathrm{E}+01$ & $-4.58436 \mathrm{E}+01$ & $-3.719 \mathrm{E}-02$ & $2.440 \mathrm{E}-02$ & $2.082 \mathrm{E}+09$ \\
\hline 1 & 3 & $-5.04159 \mathrm{E}+01$ & $-4.45972 \mathrm{E}+01$ & $-7.896 \mathrm{E}-02$ & $4.071 \mathrm{E}-02$ & $7.158 \mathrm{E}+09$ \\
\hline 1 & 4 & $-5.04159 \mathrm{E}+01$ & $-4.39490 \mathrm{E}+01$ & $-8.538 \mathrm{E}-03$ & 3.961E-03 & $9.561 \mathrm{E}+08$ \\
\hline 1 & 5 & $-5.04159 \mathrm{E}+01$ & $-2.20006 \mathrm{E}+01$ & $-5.614 \mathrm{E}-02$ & $5.927 \mathrm{E}-03$ & $1.214 \mathrm{E}+11$ \\
\hline 1 & 6 & $-5.04159 \mathrm{E}+01$ & $-2.17248 \mathrm{E}+01$ & $-3.410 \mathrm{E}-04$ & $3.566 \mathrm{E}-05$ & $7.517 \mathrm{E}+08$ \\
\hline 1 & 7 & $-5.04159 \mathrm{E}+01$ & $-1.92584 \mathrm{E}+01$ & $-8.122 \mathrm{E}-01$ & $7.820 \mathrm{E}-02$ & $2.111 \mathrm{E}+12$ \\
\hline 1 & 8 & $-5.04159 \mathrm{E}+01$ & $-1.91117 \mathrm{E}+01$ & $-3.833 \mathrm{E}-01$ & 3.673E-02 & $1.006 \mathrm{E}+12$ \\
\hline 1 & 9 & $-5.04159 \mathrm{E}+01$ & $-1.87724 \mathrm{E}+01$ & $-8.515 \mathrm{E}-03$ & 8.073E-04 & $2.283 \mathrm{E}+10$ \\
\hline 1 & 10 & $-5.04159 \mathrm{E}+01$ & $-1.86813 \mathrm{E}+01$ & $-2.095 \mathrm{E}-02$ & $1.980 \mathrm{E}-03$ & $5.649 \mathrm{E}+10$ \\
\hline 1 & 11 & $-5.04159 \mathrm{E}+01$ & $-1.86458 \mathrm{E}+01$ & $-5.138 \mathrm{E}-03$ & $4.852 \mathrm{E}-03$ & $1.389 \mathrm{E}+10$ \\
\hline 1 & 12 & $-5.04159 \mathrm{E}+01$ & $-1.84751 \mathrm{E}+01$ & $-1.566 \mathrm{E}-04$ & $1.471 \mathrm{E}-05$ & $4.278 \mathrm{E}+08$ \\
\hline 1 & 13 & $-5.04159 \mathrm{E}+01$ & $-1.82444 \mathrm{E}+01$ & $-2.107 \mathrm{E}-01$ & $1.965 \mathrm{E}-02$ & $5.839 \mathrm{E}+11$ \\
\hline 1 & 14 & $-5.04159 \mathrm{E}+01$ & $-1.79813 \mathrm{E}+01$ & $-9.334 \mathrm{E}-02$ & 8.633E-03 & $2.629 \mathrm{E}+11$ \\
\hline 1 & 15 & $-5.04159 \mathrm{E}+01$ & $-1.66700 \mathrm{E}+01$ & $-1.963 \mathrm{E}-02$ & $1.745 \mathrm{E}-03$ & $5.986 \mathrm{E}+10$ \\
\hline 1 & 16 & $-5.04159 \mathrm{E}+01$ & $-1.66046 \mathrm{E}+01$ & $-2.252 \mathrm{E}-04$ & $1.998 \mathrm{E}-05$ & $6.894 \mathrm{E}+08$ \\
\hline 1 & 17 & $-5.04159 \mathrm{E}+01$ & $-1.64673 \mathrm{E}+01$ & $-2.083 \mathrm{E}-03$ & $1.841 \mathrm{E}-04$ & $6.427 \mathrm{E}+09$ \\
\hline 1 & 18 & $-5.04159 \mathrm{E}+01$ & $-1.57979 \mathrm{E}+01$ & $-7.669 \mathrm{E}-03$ & $6.646 \mathrm{E}-04$ & $2.461 \mathrm{E}+10$ \\
\hline 1 & 19 & $-5.04159 \mathrm{E}+01$ & $-1.57593 \mathrm{E}+01$ & $-4.275 \mathrm{E}-02$ & $3.701 \mathrm{E}-03$ & $1.375 \mathrm{E}+11$ \\
\hline 1 & 20 & $-5.04159 \mathrm{E}+01$ & $-1.55895 \mathrm{E}+01$ & $-1.030 \mathrm{E}-02$ & $8.873 \mathrm{E}-04$ & $3.346 \mathrm{E}+10$ \\
\hline 1 & 21 & $-5.04159 \mathrm{E}+01$ & $-1.54751 \mathrm{E}+01$ & $-1.992 \mathrm{E}-02$ & $1.710 \mathrm{E}-03$ & $6.512 \mathrm{E}+10$ \\
\hline 1 & 22 & $-5.04159 \mathrm{E}+01$ & $-1.54647 \mathrm{E}+01$ & $-8.200 \mathrm{E}-03$ & 7.038E-04 & $2.682 \mathrm{E}+10$ \\
\hline 1 & 23 & $-5.04159 \mathrm{E}+01$ & $-1.48961 \mathrm{E}+01$ & $-2.989 \mathrm{E}-04$ & $2.525 \mathrm{E}-05$ & $1.010 \mathrm{E}+09$ \\
\hline 1 & 24 & $-5.04159 \mathrm{E}+01$ & $-1.46203 \mathrm{E}+01$ & $-5.201 \mathrm{E}-03$ & 4.359E-04 & $1.784 \mathrm{E}+10$ \\
\hline 1 & 25 & $-5.04159 \mathrm{E}+01$ & $-1.42630 \mathrm{E}+01$ & $-1.841 \mathrm{E}-03$ & $1.528 \mathrm{E}-04$ & $6.443 \mathrm{E}+09$ \\
\hline 1 & 26 & $-5.04159 \mathrm{E}+01$ & $-1.33759 \mathrm{E}+01$ & $-4.990 \mathrm{E}-04$ & $4.042 \mathrm{E}-05$ & $1.833 \mathrm{E}+09$ \\
\hline 1 & 27 & $-5.04159 \mathrm{E}+01$ & $-1.31269 \mathrm{E}+01$ & $-3.161 \mathrm{E}-05$ & $2.543 \mathrm{E}-06$ & $1.177 \mathrm{E}+08$ \\
\hline 1 & 28 & $-5.04159 \mathrm{E}+01$ & $-1.30556 \mathrm{E}+01$ & $-1.439 \mathrm{E}-06$ & $1.156 \mathrm{E}-07$ & $5.377 \mathrm{E}+06$ \\
\hline 1 & 29 & $-5.04159 \mathrm{E}+01$ & $-1.26698 \mathrm{E}+01$ & $-4.509 \mathrm{E}-03$ & $3.584 \mathrm{E}-04$ & $1.720 \mathrm{E}+10$ \\
\hline 1 & 30 & $-5.04159 \mathrm{E}+01$ & $-1.25883 \mathrm{E}+01$ & $-2.180 \mathrm{E}-03$ & $1.729 \mathrm{E}-04$ & $8.353 \mathrm{E}+09$ \\
\hline 1 & 31 & $-5.04159 \mathrm{E}+01$ & $-1.23791 \mathrm{E}+01$ & $-1.555 \mathrm{E}-03$ & $1.226 \mathrm{E}-04$ & $6.024 \mathrm{E}+09$ \\
\hline 1 & 32 & $-5.04159 \mathrm{E}+01$ & $-1.19016 \mathrm{E}+01$ & $-4.197 \mathrm{E}-03$ & $3.269 \mathrm{E}-04$ & $1.667 \mathrm{E}+10$ \\
\hline 1 & 33 & $-5.04159 \mathrm{E}+01$ & $-1.18386 \mathrm{E}+01$ & $-1.906 \mathrm{E}-03$ & $1.482 \mathrm{E}-04$ & $7.595 \mathrm{E}+09$ \\
\hline 1 & 34 & $-5.04159 \mathrm{E}+01$ & $-1.17009 \mathrm{E}+01$ & $-4.479 \mathrm{E}-03$ & $3.471 \mathrm{E}-04$ & $1.797 \mathrm{E}+10$ \\
\hline 1 & 35 & $-5.04159 \mathrm{E}+01$ & $-1.15532 \mathrm{E}+01$ & $-6.841 \mathrm{E}-04$ & $5.281 \mathrm{E}-05$ & $2.766 \mathrm{E}+09$ \\
\hline 1 & 36 & $-5.04159 \mathrm{E}+01$ & $-1.14449 \mathrm{E}+01$ & $-2.326 \mathrm{E}-03$ & $1.791 \mathrm{E}-04$ & $9.457 \mathrm{E}+09$ \\
\hline 1 & 37 & $-5.04159 \mathrm{E}+01$ & $-1.13763 \mathrm{E}+01$ & $-3.650 \mathrm{E}-03$ & $2.805 \mathrm{E}-04$ & $1.489 \mathrm{E}+10$ \\
\hline 1 & 38 & $-5.04159 \mathrm{E}+01$ & $-1.13214 \mathrm{E}+01$ & $-5.940 \mathrm{E}-03$ & $4.558 \mathrm{E}-04$ & $2.431 \mathrm{E}+10$ \\
\hline 1 & 39 & $-5.04159 \mathrm{E}+01$ & $-1.12634 \mathrm{E}+01$ & $-4.570 \mathrm{E}-02$ & $3.502 \mathrm{E}-03$ & $1.876 \mathrm{E}+11$ \\
\hline 1 & 40 & $-5.04159 \mathrm{E}+01$ & $-1.11175 \mathrm{E}+01$ & $-2.745 \mathrm{E}-02$ & $2.096 \mathrm{E}-03$ & $1.135 \mathrm{E}+11$ \\
\hline 1 & 41 & $-5.04159 \mathrm{E}+01$ & $-1.10946 \mathrm{E}+01$ & $-4.751 \mathrm{E}-03$ & $3.625 \mathrm{E}-04$ & $1.967 \mathrm{E}+10$ \\
\hline 1 & 42 & $-5.04159 \mathrm{E}+01$ & $-1.09356 \mathrm{E}+01$ & $-1.423 \mathrm{E}-02$ & $1.081 \mathrm{E}-03$ & $5.940 \mathrm{E}+10$ \\
\hline 1 & 43 & $-5.04159 \mathrm{E}+01$ & $-1.07848 \mathrm{E}+01$ & $-8.384 \mathrm{E}-02$ & $6.347 \mathrm{E}-03$ & $3.526 \mathrm{E}+11$ \\
\hline 1 & 44 & $-5.04159 \mathrm{E}+01$ & $-1.06338 \mathrm{E}+01$ & $-1.724 \mathrm{E}-01$ & $1.300 \mathrm{E}-02$ & $7.306 \mathrm{E}+11$ \\
\hline 1 & 45 & $-5.04159 \mathrm{E}+01$ & $-1.05204 \mathrm{E}+01$ & $-7.928 \mathrm{E}-03$ & $5.962 \mathrm{E}-04$ & $3.379 \mathrm{E}+10$ \\
\hline 1 & 46 & $-5.04159 \mathrm{E}+01$ & $-1.03856 \mathrm{E}+01$ & $-7.162 \mathrm{E}-05$ & $5.367 \mathrm{E}-06$ & $3.073 \mathrm{E}+08$ \\
\hline 1 & 47 & $-5.04159 \mathrm{E}+01$ & $-9.45149 \mathrm{E}+00$ & $-1.226 \mathrm{E}-03$ & $8.979 \mathrm{E}-05$ & $5.508 \mathrm{E}+09$ \\
\hline 1 & 48 & $-5.04159 \mathrm{E}+01$ & $-9.40487 \mathrm{E}+00$ & $-5.841 \mathrm{E}-05$ & $4.273 \mathrm{E}-06$ & $2.630 \mathrm{E}+08$ \\
\hline 1 & 49 & $-5.04159 \mathrm{E}+01$ & $-9.36936 \mathrm{E}+00$ & $-1.525 \mathrm{E}-06$ & $1.115 \mathrm{E}-07$ & $6.878 \mathrm{E}+06$ \\
\hline 1 & 50 & $-5.04159 \mathrm{E}+01$ & $-8.69880 \mathrm{E}+00$ & $-7.136 \mathrm{E}-06$ & $5.132 \mathrm{E}-07$ & $3.325 \mathrm{E}+07$ \\
\hline
\end{tabular}


Table 6. Oscillator strengths and transition probabilities for Fe XXI (see text for explanation)

\begin{tabular}{|c|c|c|c|c|c|c|}
\hline \multicolumn{7}{|c|}{$\begin{array}{ll}26 & 6\end{array}$} \\
\hline & 0 & 2 & & & & \\
\hline 64 & 157 & $E_{i}(\mathrm{Ry})$ & $E_{j}(\mathrm{Ry})$ & $g f_{\mathrm{L}}$ & $S$ & $A_{j i}\left(\mathrm{~s}^{-1}\right)$ \\
\hline 1 & 1 & $-1.24292 \mathrm{E}+02$ & $-1.17168 \mathrm{E}+02$ & $-8.556 \mathrm{E}-02$ & 3.603E-02 & $1.163 \mathrm{E}+10$ \\
\hline 1 & 2 & $-1.24292 \mathrm{E}+02$ & $-1.15815 \mathrm{E}+02$ & $-2.267 \mathrm{E}-02$ & 8.023E-03 & $4.362 \mathrm{E}+09$ \\
\hline 1 & 3 & $-1.24292 \mathrm{E}+02$ & $-1.14241 \mathrm{E}+02$ & $-3.606 \mathrm{E}-02$ & $1.076 \mathrm{E}-02$ & $9.752 \mathrm{E}+09$ \\
\hline 1 & 4 & $-1.24292 \mathrm{E}+02$ & $-1.12695 \mathrm{E}+02$ & $-1.038 \mathrm{E}-04$ & $2.685 \mathrm{E}-05$ & $3.738 \mathrm{E}+07$ \\
\hline 1 & 5 & $-1.24292 \mathrm{E}+02$ & $-5.45579 \mathrm{E}+01$ & $-5.459 \mathrm{E}-02$ & $2.348 \mathrm{E}-03$ & $7.108 \mathrm{E}+11$ \\
\hline 1 & 6 & $-1.24292 \mathrm{E}+02$ & $-5.36838 \mathrm{E}+01$ & $-2.443 \mathrm{E}-06$ & $1.038 \mathrm{E}-07$ & $3.261 \mathrm{E}+07$ \\
\hline 1 & 7 & $-1.24292 \mathrm{E}+02$ & $-4.98528 \mathrm{E}+01$ & $-1.097 \mathrm{E}+00$ & $4.421 \mathrm{E}-02$ & $1.628 \mathrm{E}+13$ \\
\hline 1 & 8 & $-1.24292 \mathrm{E}+02$ & $-4.92676 \mathrm{E}+01$ & $-2.376 \mathrm{E}-01$ & $9.501 \mathrm{E}-03$ & $3.581 \mathrm{E}+12$ \\
\hline 1 & 9 & $-1.24292 \mathrm{E}+02$ & $-4.89841 \mathrm{E}+01$ & $-6.728 \mathrm{E}-02$ & $2.680 \mathrm{E}-03$ & $1.022 \mathrm{E}+12$ \\
\hline 1 & 10 & $-1.24292 \mathrm{E}+02$ & $-4.87720 \mathrm{E}+01$ & $-2.967 \mathrm{E}-02$ & $1.179 \mathrm{E}-03$ & $4.530 \mathrm{E}+11$ \\
\hline 1 & 11 & $-1.24292 \mathrm{E}+02$ & $-4.84796 \mathrm{E}+01$ & $-2.779 \mathrm{E}-02$ & $1.100 \mathrm{E}-03$ & $4.277 \mathrm{E}+11$ \\
\hline 1 & 12 & $-1.24292 \mathrm{E}+02$ & $-4.79949 \mathrm{E}+01$ & $-4.288 \mathrm{E}-02$ & $1.686 \mathrm{E}-03$ & $6.684 \mathrm{E}+11$ \\
\hline 1 & 13 & $-1.24292 \mathrm{E}+02$ & $-4.78167 \mathrm{E}+01$ & $-1.718 \mathrm{E}-01$ & $6.739 \mathrm{E}-03$ & $2.690 \mathrm{E}+12$ \\
\hline 1 & 14 & $-1.24292 \mathrm{E}+02$ & $-4.69872 \mathrm{E}+01$ & $-2.536 \mathrm{E}-02$ & $9.842 \mathrm{E}-04$ & $4.058 \mathrm{E}+11$ \\
\hline 1 & 15 & $-1.24292 \mathrm{E}+02$ & $-4.57908 \mathrm{E}+01$ & $-3.615 \mathrm{E}-02$ & $1.382 \mathrm{E}-03$ & $5.965 \mathrm{E}+11$ \\
\hline 1 & 16 & $-1.24292 \mathrm{E}+02$ & $-4.51108 \mathrm{E}+01$ & $-4.164 \mathrm{E}-03$ & $1.578 \mathrm{E}-04$ & $6.990 \mathrm{E}+10$ \\
\hline 1 & 17 & $-1.24292 \mathrm{E}+02$ & $-4.48241 \mathrm{E}+01$ & $-1.864 \mathrm{E}-03$ & 7.037E-05 & $3.151 \mathrm{E}+10$ \\
\hline 1 & 18 & $-1.24292 \mathrm{E}+02$ & $-4.43456 \mathrm{E}+01$ & $-1.420 \mathrm{E}-03$ & $5.329 \mathrm{E}-05$ & $2.431 \mathrm{E}+10$ \\
\hline 1 & 19 & $-1.24292 \mathrm{E}+02$ & $-4.40629 \mathrm{E}+01$ & $-1.372 \mathrm{E}-02$ & $5.130 \mathrm{E}-04$ & $2.364 \mathrm{E}+11$ \\
\hline 1 & 20 & $-1.24292 \mathrm{E}+02$ & $-4.38519 \mathrm{E}+01$ & $-1.681 \mathrm{E}-02$ & $6.269 \mathrm{E}-04$ & $2.912 \mathrm{E}+11$ \\
\hline 1 & 21 & $-1.24292 \mathrm{E}+02$ & $-4.33769 \mathrm{E}+01$ & $-4.298 \mathrm{E}-02$ & $1.594 \mathrm{E}-03$ & $7.534 \mathrm{E}+11$ \\
\hline 1 & 22 & $-1.24292 \mathrm{E}+02$ & $-4.26163 \mathrm{E}+01$ & $-1.653 \mathrm{E}-03$ & $6.072 \mathrm{E}-05$ & $2.953 \mathrm{E}+10$ \\
\hline 1 & 23 & $-1.24292 \mathrm{E}+02$ & $-4.22948 \mathrm{E}+01$ & $-7.745 \mathrm{E}-03$ & $2.834 \mathrm{E}-04$ & $1.394 \mathrm{E}+11$ \\
\hline 1 & 24 & $-1.24292 \mathrm{E}+02$ & $-4.18241 \mathrm{E}+01$ & $-1.151 \mathrm{E}-02$ & $4.187 \mathrm{E}-04$ & $2.096 \mathrm{E}+11$ \\
\hline 1 & 25 & $-1.24292 \mathrm{E}+02$ & $-4.13257 \mathrm{E}+01$ & $-9.296 \mathrm{E}-04$ & $3.361 \mathrm{E}-05$ & $1.713 \mathrm{E}+10$ \\
\hline 1 & 26 & $-1.24292 \mathrm{E}+02$ & $-3.98368 \mathrm{E}+01$ & $-8.523 \mathrm{E}-04$ & $3.028 \mathrm{E}-05$ & $1.628 \mathrm{E}+10$ \\
\hline 1 & 27 & $-1.24292 \mathrm{E}+02$ & $-3.91261 \mathrm{E}+01$ & $-5.482 \mathrm{E}-07$ & $1.931 \mathrm{E}-08$ & $1.065 \mathrm{E}+07$ \\
\hline 1 & 28 & $-1.24292 \mathrm{E}+02$ & $-3.89117 \mathrm{E}+01$ & $-2.576 \mathrm{E}-06$ & $9.051 \mathrm{E}-08$ & $5.028 \mathrm{E}+07$ \\
\hline 1 & 29 & $-1.24292 \mathrm{E}+02$ & $-3.81248 \mathrm{E}+01$ & $-3.895 \mathrm{E}-03$ & $1.356 \mathrm{E}-04$ & $7.743 \mathrm{E}+10$ \\
\hline 1 & 30 & $-1.24292 \mathrm{E}+02$ & $-3.72353 \mathrm{E}+01$ & $-3.496 \mathrm{E}-03$ & $1.205 \mathrm{E}-04$ & $7.093 \mathrm{E}+10$ \\
\hline 1 & 31 & $-1.24292 \mathrm{E}+02$ & $-3.68057 \mathrm{E}+01$ & $-3.001 \mathrm{E}-05$ & $1.029 \mathrm{E}-06$ & $6.150 \mathrm{E}+08$ \\
\hline 1 & 32 & $-1.24292 \mathrm{E}+02$ & $-3.66023 \mathrm{E}+01$ & $-2.169 \mathrm{E}-03$ & $7.420 \mathrm{E}-05$ & $4.465 \mathrm{E}+10$ \\
\hline 1 & 33 & $-1.24292 \mathrm{E}+02$ & $-3.62569 \mathrm{E}+01$ & $-1.857 \mathrm{E}-03$ & $6.328 \mathrm{E}-05$ & $3.853 \mathrm{E}+10$ \\
\hline 1 & 34 & $-1.24292 \mathrm{E}+02$ & $-3.56225 \mathrm{E}+01$ & $-6.271 \mathrm{E}-04$ & $2.122 \mathrm{E}-05$ & $1.320 \mathrm{E}+10$ \\
\hline 1 & 35 & $-1.24292 \mathrm{E}+02$ & $-3.52504 \mathrm{E}+01$ & $-4.418 \mathrm{E}-04$ & $1.489 \mathrm{E}-05$ & $9.379 \mathrm{E}+09$ \\
\hline 1 & 36 & $-1.24292 \mathrm{E}+02$ & $-3.41344 \mathrm{E}+01$ & $-2.004 \mathrm{E}-04$ & $6.668 \mathrm{E}-06$ & $4.361 \mathrm{E}+09$ \\
\hline 1 & 37 & $-1.24292 \mathrm{E}+02$ & $-2.96152 \mathrm{E}+01$ & $-3.262 \mathrm{E}-03$ & $1.034 \mathrm{E}-04$ & $7.829 \mathrm{E}+10$ \\
\hline 1 & 38 & $-1.24292 \mathrm{E}+02$ & $-2.86486 \mathrm{E}+01$ & $-5.945 \mathrm{E}-03$ & $1.865 \mathrm{E}-04$ & $1.456 \mathrm{E}+11$ \\
\hline 1 & 39 & $-1.24292 \mathrm{E}+02$ & $-2.78866 \mathrm{E}+01$ & $-1.027 \mathrm{E}-01$ & $3.196 \mathrm{E}-03$ & $2.556 \mathrm{E}+12$ \\
\hline 1 & 40 & $-1.24292 \mathrm{E}+02$ & $-2.69896 \mathrm{E}+01$ & $-1.375 \mathrm{E}-01$ & $4.239 \mathrm{E}-03$ & $3.485 \mathrm{E}+12$ \\
\hline 1 & 41 & $-1.24292 \mathrm{E}+02$ & $-2.67722 \mathrm{E}+01$ & $-5.394 \mathrm{E}-03$ & $1.659 \mathrm{E}-04$ & $1.373 \mathrm{E}+11$ \\
\hline 1 & 42 & $-1.24292 \mathrm{E}+02$ & $-2.51918 \mathrm{E}+01$ & $-1.245 \mathrm{E}-03$ & $3.769 \mathrm{E}-05$ & $3.273 \mathrm{E}+10$ \\
\hline 1 & 43 & $-1.24292 \mathrm{E}+02$ & $-2.48551 \mathrm{E}+01$ & $-3.036 \mathrm{E}-02$ & $9.160 \mathrm{E}-02$ & $8.038 \mathrm{E}+11$ \\
\hline 1 & 44 & $-1.24292 \mathrm{E}+02$ & $-2.47189 \mathrm{E}+01$ & $-6.847 \mathrm{E}-04$ & $2.063 \mathrm{E}-05$ & $1.818 \mathrm{E}+10$ \\
\hline 1 & 45 & $-1.24292 \mathrm{E}+02$ & $-2.44906 \mathrm{E}+01$ & $-1.546 \mathrm{E}-02$ & $4.647 \mathrm{E}-04$ & $4.124 \mathrm{E}+11$ \\
\hline 1 & 46 & $-1.24292 \mathrm{E}+02$ & $-2.40150 \mathrm{E}+01$ & $-5.308 \mathrm{E}-02$ & $1.588 \mathrm{E}-03$ & $1.429 \mathrm{E}+12$ \\
\hline 1 & 47 & $-1.24292 \mathrm{E}+02$ & $-2.33917 \mathrm{E}+01$ & $-9.461 \mathrm{E}-06$ & $2.813 \mathrm{E}-07$ & $2.579 \mathrm{E}+08$ \\
\hline 1 & 48 & $-1.24292 \mathrm{E}+02$ & $-2.29626 \mathrm{E}+01$ & $-1.697 \mathrm{E}-06$ & $5.024 \mathrm{E}-08$ & $4.664 \mathrm{E}+07$ \\
\hline 1 & 49 & $-1.24292 \mathrm{E}+02$ & $-2.29205 \mathrm{E}+01$ & $-3.144 \mathrm{E}-05$ & $9.304 \mathrm{E}-07$ & $8.651 \mathrm{E}+08$ \\
\hline 1 & 50 & $-1.24292 \mathrm{E}+02$ & $-2.20492 \mathrm{E}+01$ & $-4.263 \mathrm{E}-03$ & $1.251 \mathrm{E}-04$ & $1.193 \mathrm{E}+11$ \\
\hline
\end{tabular}


Table 7. a. Sample set of reprocessed $f$ - and $A$-values for Ar XIII using observed transition energies. The negative sign for the energies is omitted for convenience. $I_{i}$ and $I_{j}$ are the level indices

\begin{tabular}{|c|c|c|c|c|c|c|c|c|c|}
\hline$C_{i}$ & $C_{j}$ & $S_{i} L_{i} \pi_{i}$ & $S_{j} L_{j} \pi_{j}$ & $g_{i}\left(I_{i}\right)$ & $g_{j}\left(I_{j}\right)$ & $E_{i}(\mathrm{Ry})$ & $E_{j}(\mathrm{Ry})$ & $f$ & $A_{j i}\left(\mathrm{~s}^{-1}\right)$ \\
\hline $2 \mathrm{~s}^{2} 2 \mathrm{p}^{2}$ & $-2 \mathrm{~s} 2 \mathrm{p}^{3}$ & ${ }^{3} \mathrm{P}^{\mathrm{e}}$ & ${ }^{3} \mathrm{D}^{\circ}$ & $1(1)$ & $3(1)$ & 50.3320 & 46.4760 & $7.370 \mathrm{E}-02$ & $2.93 \mathrm{E}+09$ \\
\hline $2 \mathrm{~s}^{2} 2 \mathrm{p}^{2}$ & $-2 \mathrm{~s} 2 \mathrm{p}^{3}$ & ${ }^{3} \mathrm{P}^{\mathrm{e}}$ & ${ }^{3} \mathrm{P}^{\mathrm{o}}$ & $1(1)$ & $3(2)$ & 50.3320 & 45.8140 & $3.675 \mathrm{E}-02$ & $2.01 \mathrm{E}+09$ \\
\hline $2 \mathrm{~s}^{2} 2 \mathrm{p}^{2}$ & $-2 \mathrm{~s} 2 \mathrm{p}^{3}$ & ${ }^{3} \mathrm{P}^{\mathrm{e}}$ & ${ }^{3} \mathrm{~S}^{\circ}$ & $1(1)$ & $3(3)$ & 50.3320 & 44.6040 & $7.773 \mathrm{E}-02$ & $6.83 \mathrm{E}+09$ \\
\hline $2 s^{2} 2 p^{2}$ & $-2 \mathrm{~s} 2 \mathrm{p}^{3}$ & ${ }^{3} \mathrm{P}^{\mathrm{e}}$ & ${ }^{1} \mathrm{P}^{\mathrm{o}}$ & $1(1)$ & $3(4)$ & 50.3320 & 43.9660 & $8.405 \mathrm{E}-03$ & $9.12 \mathrm{E}+08$ \\
\hline $2 s^{2} 2 p^{2}$ & $-2 s^{2} 2 p 3 s$ & ${ }^{3} \mathrm{P}^{\mathrm{e}}$ & ${ }^{3} \mathrm{P}^{\mathrm{o}}$ & $1(1)$ & $3(5)$ & 50.3320 & 21.6590 & $5.665 \mathrm{E}-02$ & $1.25 \mathrm{E}+11$ \\
\hline $2 \mathrm{~s}^{2} 2 \mathrm{p}^{2}$ & $-2 s^{2} 2 p 3 s$ & ${ }^{3} \mathrm{P}^{\mathrm{e}}$ & ${ }^{1} \mathrm{P}^{\mathrm{o}}$ & $1(1)$ & $3(6)$ & 50.3320 & 21.3810 & $3.441 \mathrm{E}-04$ & $7.72 \mathrm{E}+08$ \\
\hline $2 \mathrm{~s}^{2} 2 \mathrm{p}^{2}$ & $-2 \mathrm{~s}^{2} 2 \mathrm{p} 3 \mathrm{~d}$ & ${ }^{3} \mathrm{P}^{\mathrm{e}}$ & ${ }^{3} \mathrm{D}^{\mathrm{o}}$ & $1(1)$ & $3(7)$ & 50.3320 & 19.2530 & $8.101 \mathrm{E}-01$ & $2.10 \mathrm{E}+12$ \\
\hline $2 s^{2} 2 p^{2}$ & $-2 \mathrm{~s}^{2} 2 \mathrm{p} 3 \mathrm{~d}$ & ${ }^{3} \mathrm{P}^{\mathrm{e}}$ & ${ }^{3} \mathrm{P}^{\mathrm{o}}$ & $1(1)$ & $3(8)$ & 50.3320 & 19.0370 & $3.832 \mathrm{E}-01$ & $1.00 \mathrm{E}+12$ \\
\hline $2 s^{2} 2 p^{2}$ & $-2 \mathrm{~s}^{2} 2 \mathrm{p} 3 \mathrm{~d}$ & ${ }^{3} \mathrm{P}^{\mathrm{e}}$ & ${ }^{1} \mathrm{P}^{\mathrm{o}}$ & $1(1)$ & $3(9)$ & 50.3320 & 18.7400 & $8.501 \mathrm{E}-03$ & $2.27 \mathrm{E}+10$ \\
\hline $2 \mathrm{~s}^{2} 2 \mathrm{p}^{2}$ & $-2 \mathrm{~s} 2 \mathrm{p}^{2} 3 \mathrm{p}$ & ${ }^{3} \mathrm{P}^{\mathrm{e}}$ & ${ }^{3} \mathrm{D}^{\mathrm{o}}$ & $1(1)$ & $3(16)$ & 50.3320 & 17.1260 & $2.212 \mathrm{E}-04$ & $6.53 \mathrm{E}+08$ \\
\hline $2 \mathrm{~s}^{2} 2 \mathrm{p}^{2}$ & $-2 \mathrm{~s} 2 \mathrm{p}^{2} 3 \mathrm{p}$ & ${ }^{3} \mathrm{P}^{\mathrm{e}}$ & ${ }^{3} \mathrm{P}^{\mathrm{o}}$ & $1(1)$ & $3(18)$ & 50.3320 & 15.7840 & $7.654 \mathrm{E}-03$ & $2.45 \mathrm{E}+10$ \\
\hline $2 s^{2} 2 p^{2}$ & $-2 \mathrm{~s} 2 \mathrm{p}^{2} 3 \mathrm{p}$ & ${ }^{3} \mathrm{P}^{\mathrm{e}}$ & ${ }^{1} \mathrm{P}^{\mathrm{o}}$ & $1(1)$ & $3(20)$ & 50.3320 & 15.5810 & $1.028 \mathrm{E}-02$ & $3.32 \mathrm{E}+10$ \\
\hline $2 s^{2} 2 p^{2}$ & $-2 \mathrm{~s} 2 \mathrm{p}^{2} 3 \mathrm{p}$ & ${ }^{3} \mathrm{P}^{\mathrm{e}}$ & ${ }^{3} \mathrm{~S}^{\circ}$ & $1(1)$ & $3(21)$ & 50.3320 & 15.5630 & $1.982 \mathrm{E}-02$ & $6.41 \mathrm{E}+10$ \\
\hline $2 s^{2} 2 p^{2}$ & $-2 s^{2} 2 p 4 s$ & ${ }^{3} \mathrm{P}^{\mathrm{e}}$ & ${ }^{3} \mathrm{P}^{\mathrm{o}}$ & $1(1)$ & $3(30)$ & 50.3320 & 11.5800 & $2.233 \mathrm{E}-03$ & $8.98 \mathrm{E}+09$ \\
\hline $2 s^{2} 2 p^{2}$ & $-2 s^{2} 2 p 4 s$ & ${ }^{3} \mathrm{P}^{\mathrm{e}}$ & ${ }^{1} \mathrm{P}^{\mathrm{o}}$ & $1(1)$ & $3(31)$ & 50.3320 & 11.8260 & $1.574 \mathrm{E}-03$ & $6.25 \mathrm{E}+09$ \\
\hline $2 s^{2} 2 p^{2}$ & $-2 \mathrm{~s}^{2} 2 \mathrm{p} 4 \mathrm{~d}$ & ${ }^{3} \mathrm{P}^{\mathrm{e}}$ & ${ }^{3} \mathrm{P}^{\mathrm{o}}$ & $1(1)$ & $3(43)$ & 50.3320 & 10.5650 & $8.413 \mathrm{E}-02$ & $3.56 \mathrm{E}+11$ \\
\hline $2 \mathrm{~s}^{2} 2 \mathrm{p}^{2}$ & $-2 \mathrm{~s}^{2} 2 \mathrm{p} 4 \mathrm{~d}$ & ${ }^{3} \mathrm{P}^{\mathrm{e}}$ & ${ }^{1} \mathrm{P}^{\mathrm{o}}$ & $1(1)$ & $3(45)$ & 50.3320 & 10.7740 & $7.861 \mathrm{E}-03$ & $3.29 \mathrm{E}+10$ \\
\hline $2 \mathrm{~s}^{2} 2 \mathrm{p}^{2}$ & $-2 \mathrm{~s} 2 \mathrm{p}^{3}$ & ${ }^{1} \mathrm{~S}^{\mathrm{e}}$ & ${ }^{3} \mathrm{D}^{\mathrm{o}}$ & $1(2)$ & $3(1)$ & 48.8560 & 46.4760 & $2.257 \mathrm{E}-04$ & $3.42 \mathrm{E}+06$ \\
\hline $2 \mathrm{~s}^{2} 2 \mathrm{p}^{2}$ & $-2 \mathrm{~s} 2 \mathrm{p}^{3}$ & ${ }^{1} \mathrm{~S}^{\mathrm{e}}$ & ${ }^{3} \mathrm{P}^{\mathrm{o}}$ & $1(2)$ & $3(2)$ & 48.8560 & 45.8140 & $4.438 \mathrm{E}-04$ & $1.10 \mathrm{E}+07$ \\
\hline $2 \mathrm{~s}^{2} 2 \mathrm{p}^{2}$ & $-2 \mathrm{~s} 2 \mathrm{p}^{3}$ & ${ }^{1} \mathrm{~S}^{\mathrm{e}}$ & ${ }^{3} \mathrm{~S}^{\circ}$ & $1(2)$ & $3(3)$ & 48.8560 & 44.6040 & $8.290 \mathrm{E}-03$ & $4.01 \mathrm{E}+08$ \\
\hline $2 \mathrm{~s}^{2} 2 \mathrm{p}^{2}$ & $-2 \mathrm{~s} 2 \mathrm{p}^{3}$ & ${ }^{1} \mathrm{~S}^{\mathrm{e}}$ & ${ }^{1} \mathrm{P}^{\mathrm{o}}$ & $1(2)$ & $3(4)$ & 48.8560 & 43.9660 & $1.314 \mathrm{E}-01$ & $8.41 \mathrm{E}+09$ \\
\hline $2 s^{2} 2 p^{2}$ & $-2 s^{2} 2 p 3 s$ & ${ }^{1} \mathrm{~S}^{\mathrm{e}}$ & ${ }^{3} \mathrm{P}^{\mathrm{o}}$ & $1(2)$ & $3(5)$ & 48.8560 & 21.6590 & $8.977 \mathrm{E}-04$ & $1.78 \mathrm{E}+09$ \\
\hline $2 s^{2} 2 p^{2}$ & $-2 s^{2} 2 p 3 s$ & ${ }^{1} \mathrm{~S}^{\mathrm{e}}$ & ${ }^{1} \mathrm{P}^{\mathrm{o}}$ & $1(2)$ & $3(6)$ & 48.8560 & 21.3810 & $7.492 \mathrm{E}-02$ & $1.51 \mathrm{E}+11$ \\
\hline $2 \mathrm{~s}^{2} 2 \mathrm{p}^{2}$ & $-2 \mathrm{~s}^{2} 2 \mathrm{p} 3 \mathrm{~d}$ & ${ }^{1} \mathrm{~S}^{\mathrm{e}}$ & ${ }^{3} \mathrm{D}^{\mathrm{o}}$ & $1(2)$ & $3(7)$ & 48.8560 & 19.2530 & $8.055 \mathrm{E}-03$ & $1.89 \mathrm{E}+10$ \\
\hline $2 \mathrm{~s}^{2} 2 \mathrm{p}^{2}$ & $-2 \mathrm{~s}^{2} 2 \mathrm{p} 3 \mathrm{~d}$ & ${ }^{1} \mathrm{~S}^{\mathrm{e}}$ & ${ }^{3} \mathrm{P}^{\mathrm{o}}$ & $1(2)$ & $3(8)$ & 48.8560 & 19.0370 & $1.741 \mathrm{E}-03$ & $4.15 \mathrm{E}+09$ \\
\hline $2 \mathrm{~s}^{2} 2 \mathrm{p}^{2}$ & $-2 \mathrm{~s}^{2} 2 \mathrm{p} 3 \mathrm{~d}$ & ${ }^{1} \mathrm{~S}^{\mathrm{e}}$ & ${ }^{1} \mathrm{P}^{\mathrm{o}}$ & $1(2)$ & $3(9)$ & 48.8560 & 18.7400 & $1.227 \mathrm{E}+00$ & $2.98 \mathrm{E}+12$ \\
\hline $2 \mathrm{~s}^{2} 2 \mathrm{p}^{2}$ & $-2 \mathrm{~s} 2 \mathrm{p}^{2} 3 \mathrm{p}$ & ${ }^{1} \mathrm{~S}^{\mathrm{e}}$ & ${ }^{3} \mathrm{D}^{\mathrm{o}}$ & $1(2)$ & $3(16)$ & 48.8560 & 17.1260 & 8.095E-03 & $2.18 \mathrm{E}+10$ \\
\hline $2 \mathrm{~s}^{2} 2 \mathrm{p}^{2}$ & $-2 \mathrm{~s} 2 \mathrm{p}^{2} 3 \mathrm{p}$ & ${ }^{1} \mathrm{~S}^{\mathrm{e}}$ & ${ }^{3} \mathrm{P}^{\mathrm{o}}$ & $1(2)$ & 3 (18) & 48.8560 & 15.7840 & $4.672 \mathrm{E}-02$ & $1.37 \mathrm{E}+11$ \\
\hline $2 s^{2} 2 p^{2}$ & $-2 \mathrm{~s} 2 \mathrm{p}^{2} 3 \mathrm{p}$ & ${ }^{1} \mathrm{~S}^{\mathrm{e}}$ & ${ }^{1} \mathrm{P}^{\mathrm{o}}$ & $1(2)$ & $3(20)$ & 48.8560 & 15.5810 & $2.188 \mathrm{E}-01$ & $6.49 \mathrm{E}+11$ \\
\hline $2 \mathrm{~s}^{2} 2 \mathrm{p}^{2}$ & $-2 \mathrm{~s} 2 \mathrm{p}^{2} 3 \mathrm{p}$ & ${ }^{1} \mathrm{~S}^{\mathrm{e}}$ & ${ }^{3} \mathrm{~S}^{\circ}$ & $1(2)$ & $3(21)$ & 48.8560 & 15.5630 & $6.007 \mathrm{E}-04$ & $1.78 \mathrm{E}+09$ \\
\hline $2 \mathrm{~s}^{2} 2 \mathrm{p}^{2}$ & $-2 s^{2} 2 p 4 s$ & ${ }^{1} \mathrm{~S}^{\mathrm{e}}$ & ${ }^{3} \mathrm{P}^{\mathrm{o}}$ & $1(2)$ & $3(30)$ & 48.8560 & 11.5800 & $2.184 \mathrm{E}-03$ & $8.13 \mathrm{E}+09$ \\
\hline $2 s^{2} 2 p^{2}$ & $-2 s^{2} 2 p 4 s$ & ${ }^{1} \mathrm{~S}^{\mathrm{e}}$ & ${ }^{1} \mathrm{P}^{\mathrm{o}}$ & $1(2)$ & $3(31)$ & 48.8560 & 11.8260 & $3.867 \mathrm{E}-03$ & $1.42 \mathrm{E}+10$ \\
\hline $2 \mathrm{~s}^{2} 2 \mathrm{p}^{2}$ & $-2 \mathrm{~s}^{2} 2 \mathrm{p} 4 \mathrm{~d}$ & ${ }^{1} \mathrm{~S}^{\mathrm{e}}$ & ${ }^{3} \mathrm{P}^{\mathrm{o}}$ & $1(2)$ & $3(43)$ & 48.8560 & 10.5650 & $2.516 \mathrm{E}-02$ & $9.88 \mathrm{E}+10$ \\
\hline $2 s^{2} 2 p^{2}$ & $-2 \mathrm{~s}^{2} 2 \mathrm{p} 4 \mathrm{~d}$ & ${ }^{1} \mathrm{~S}^{\mathrm{e}}$ & ${ }^{1} \mathrm{P}^{\mathrm{o}}$ & $1(2)$ & $3(45)$ & 48.8560 & 10.7740 & $2.355 \mathrm{E}-01$ & $9.14 \mathrm{E}+11$ \\
\hline $2 \mathrm{~S} 2 \mathrm{p}^{3}$ & $-2 p^{4}$ & ${ }^{3} \mathrm{D}^{\circ}$ & ${ }^{3} \mathrm{P}^{\mathrm{e}}$ & $3(1)$ & $1(3)$ & 46.4760 & 41.2420 & $3.321 \mathrm{E}-02$ & $2.19 \mathrm{E}+10$ \\
\hline $2 \mathrm{~s} 2 \mathrm{p}^{3}$ & $-2 p^{4}$ & ${ }^{3} \mathrm{P}^{\mathrm{o}}$ & ${ }^{3} \mathrm{P}^{\mathrm{e}}$ & $3(2)$ & $1(3)$ & 45.8140 & 41.2420 & $2.436 \mathrm{E}-02$ & $1.23 \mathrm{E}+10$ \\
\hline $2 \mathrm{~s} 2 \mathrm{p}^{3}$ & $-2 \mathrm{p}^{4}$ & ${ }^{3} \mathrm{~S}^{\circ}$ & ${ }^{3} \mathrm{P}^{\mathrm{e}}$ & $3(3)$ & $1(3)$ & 44.6040 & 41.2420 & $1.657 \mathrm{E}-02$ & $4.51 \mathrm{E}+09$ \\
\hline $2 \mathrm{~s} 2 \mathrm{p}^{3}$ & $-2 p^{4}$ & ${ }^{1} \mathrm{P}^{\mathrm{o}}$ & ${ }^{3} \mathrm{P}^{\mathrm{e}}$ & $3(4)$ & $1(3)$ & 43.9660 & 41.2420 & $1.568 \mathrm{E}-03$ & $2.80 \mathrm{E}+08$ \\
\hline $2 p^{4}$ & $-2 \mathrm{~s}^{2} 2 \mathrm{p} 3 \mathrm{~s}$ & ${ }^{3} \mathrm{P}^{\mathrm{e}}$ & ${ }^{3} \mathrm{P}^{\mathrm{o}}$ & $1(3)$ & $3(5)$ & 41.2420 & 21.6590 & $2.841 \mathrm{E}-06$ & $2.92 \mathrm{E}+06$ \\
\hline $2 p^{4}$ & $-2 s^{2} 2 p 3 s$ & ${ }^{3} \mathrm{P}^{\mathrm{e}}$ & ${ }^{1} \mathrm{P}^{\mathrm{o}}$ & $1(3)$ & $3(6)$ & 41.2420 & 21.3810 & $9.904 \mathrm{E}-07$ & $1.05 \mathrm{E}+06$ \\
\hline $2 p^{4}$ & $-2 \mathrm{~s}^{2} 2 \mathrm{p} 3 \mathrm{~d}$ & ${ }^{3} \mathrm{P}^{\mathrm{e}}$ & ${ }^{3} \mathrm{D}^{\circ}$ & $1(3)$ & $3(7)$ & 41.2420 & 19.2530 & $7.887 \mathrm{E}-06$ & $1.02 \mathrm{E}+07$ \\
\hline $2 \mathrm{p}^{4}$ & $-2 \mathrm{~s}^{2} 2 \mathrm{p} 3 \mathrm{~d}$ & ${ }^{3} \mathrm{P}^{\mathrm{e}}$ & ${ }^{3} \mathrm{P}^{\mathrm{o}}$ & $1(3)$ & $3(8)$ & 41.2420 & 19.0370 & $2.720 \mathrm{E}-05$ & $3.59 \mathrm{E}+07$ \\
\hline $2 p^{4}$ & $-2 \mathrm{~s}^{2} 2 \mathrm{p} 3 \mathrm{~d}$ & ${ }^{3} \mathrm{P}^{\mathrm{e}}$ & ${ }^{1} \mathrm{P}^{\mathrm{o}}$ & $1(3)$ & $3(9)$ & 41.2420 & 18.7400 & $6.594 \mathrm{E}-06$ & $8.94 \mathrm{E}+06$ \\
\hline $2 p^{4}$ & $-2 \mathrm{~s} 2 \mathrm{p}^{2} 3 \mathrm{p}$ & ${ }^{3} \mathrm{P}^{\mathrm{e}}$ & ${ }^{3} \mathrm{D}^{\circ}$ & $1(3)$ & $3(16)$ & 41.2420 & 17.1260 & $9.606 \mathrm{E}-09$ & $1.50 \mathrm{E}+04$ \\
\hline $2 p^{4}$ & $-2 \mathrm{~s} 2 \mathrm{p}^{2} 3 \mathrm{p}$ & ${ }^{3} \mathrm{P}^{\mathrm{e}}$ & ${ }^{3} \mathrm{P}^{\mathrm{o}}$ & $1(3)$ & $3(18)$ & 41.2420 & 15.7840 & $4.387 \mathrm{E}-04$ & $7.61 \mathrm{E}+08$ \\
\hline $2 \mathrm{p}^{4}$ & $-2 \mathrm{~s} 2 \mathrm{p}^{2} 3 \mathrm{p}$ & ${ }^{3} \mathrm{P}^{\mathrm{e}}$ & ${ }^{1} \mathrm{P}^{\mathrm{o}}$ & $1(3)$ & $3(20)$ & 41.2420 & 15.5810 & $5.815 \mathrm{E}-04$ & $1.03 \mathrm{E}+09$ \\
\hline $2 p^{4}$ & $-2 \mathrm{~s} 2 \mathrm{p}^{2} 3 \mathrm{p}$ & ${ }^{3} \mathrm{P}^{\mathrm{e}}$ & ${ }^{3} \mathrm{~S}^{\mathrm{o}}$ & $1(3)$ & $3(21)$ & 41.2420 & 15.5630 & $1.354 \mathrm{E}-02$ & $2.39 \mathrm{E}+10$ \\
\hline $2 p^{4}$ & $-2 \mathrm{~s}^{2} 2 \mathrm{p} 4 \mathrm{~s}$ & ${ }^{3} \mathrm{P}^{\mathrm{e}}$ & ${ }^{3} \mathrm{P}^{\mathrm{o}}$ & $1(3)$ & $3(30)$ & 41.2420 & 11.5800 & $3.678 \mathrm{E}-07$ & $8.66 \mathrm{E}+05$ \\
\hline $2 p^{4}$ & $-2 s^{2} 2 p 4 s$ & ${ }^{3} \mathrm{P}^{\mathrm{e}}$ & ${ }^{1} \mathrm{P}^{\mathrm{o}}$ & $1(3)$ & $3(31)$ & 41.2420 & 11.8260 & $1.251 \mathrm{E}-05$ & $2.90 \mathrm{E}+07$ \\
\hline $2 p^{4}$ & $-2 \mathrm{~s}^{2} 2 \mathrm{p} 4 \mathrm{~d}$ & ${ }^{3} \mathrm{P}^{\mathrm{e}}$ & ${ }^{3} \mathrm{P}^{\mathrm{o}}$ & $1(3)$ & $3(43)$ & 41.2420 & 10.5650 & $1.511 \mathrm{E}-02$ & $3.81 \mathrm{E}+10$ \\
\hline $2 \mathrm{p}^{4}$ & $-2 \mathrm{~s}^{2} 2 \mathrm{p} 4 \mathrm{~d}$ & ${ }^{3} \mathrm{P}^{\mathrm{e}}$ & ${ }^{1} \mathrm{P}^{\mathrm{o}}$ & $1(3)$ & $3(45)$ & 41.2420 & 10.7740 & $4.366 \mathrm{E}-04$ & $1.09 \mathrm{E}+09$ \\
\hline $2 \mathrm{~s}^{2} 2 \mathrm{p}^{2}$ & $-2 \mathrm{~s} 2 \mathrm{p}^{3}$ & ${ }^{3} \mathrm{P}^{\mathrm{e}}$ & ${ }^{3} \mathrm{P}^{\mathrm{o}}$ & $3(1)$ & $1(1)$ & 50.2430 & 45.8170 & $2.071 \mathrm{E}-02$ & $9.78 \mathrm{E}+09$ \\
\hline $2 \mathrm{~s} 2 \mathrm{p}^{3}$ & $-2 p^{4}$ & ${ }^{3} \mathrm{P}^{\mathrm{o}}$ & ${ }^{3} \mathrm{P}^{\mathrm{e}}$ & $1(1)$ & $3(2)$ & 45.8170 & 41.2860 & $4.223 \mathrm{E}-02$ & $2.32 \mathrm{E}+09$ \\
\hline $2 s^{2} 2 p^{2}$ & $-2 s^{2} 2 p 3 s$ & ${ }^{3} \mathrm{P}^{\mathrm{e}}$ & ${ }^{3} \mathrm{P}^{\mathrm{o}}$ & $3(1)$ & $1(2)$ & 50.2430 & 21.7220 & $1.874 \mathrm{E}-02$ & $3.67 \mathrm{E}+11$ \\
\hline $2 p^{4}$ & $-2 s^{2} 2 p 3 s$ & ${ }^{3} \mathrm{P}^{\mathrm{e}}$ & ${ }^{3} \mathrm{P}^{\mathrm{o}}$ & $3(2)$ & $1(2)$ & 41.2860 & 21.7220 & $7.065 \mathrm{E}-07$ & $6.52 \mathrm{E}+06$ \\
\hline
\end{tabular}


Table 7. b. Fine structure transitions of Ar XIII are ordered in $L S$ multiplets and are compared with previous values. Notation $a \pm b$ means $a \times 10^{b}$, "O" means for others and "P" for present. The last line of a set of dipole allowed transitions corresponds to the LS multiplet

\begin{tabular}{|c|c|c|c|c|c|c|c|c|c|}
\hline$C_{i}$ & $C_{j}$ & $S_{i} L_{i} \pi_{i}$ & $S_{j} L_{j} \pi_{j}$ & $g_{i}\left(I_{i}\right)$ & $g_{j}\left(I_{j}\right)$ & $f_{i j}(O)$ & $f_{i j}(P)$ & $A_{j i}\left(\mathrm{~s}^{-1}, O\right)$ & $A_{j i}\left(\mathrm{~s}^{-1}, P\right)$ \\
\hline $2 \mathrm{~s} 22 \mathrm{p} 2$ & $-2 \mathrm{~s} 2 \mathrm{p} 3$ & ${ }^{3} \mathrm{P} 0$ & ${ }^{3} \mathrm{D} 1$ & $1(1)$ & $3(1)$ & $8.267-2^{1}$ & $7.370-2$ & & \\
\hline $2 \mathrm{~s} 22 \mathrm{p} 2$ & $-2 \mathrm{~s} 2 \mathrm{p} 3$ & ${ }^{3} \mathrm{P} 0$ & ${ }^{3} \mathrm{D} 1$ & $3(1)$ & $3(1)$ & $1.163-2^{1}$ & 1.078-2 & & \\
\hline $2 \mathrm{~s} 22 \mathrm{p} 2$ & $-2 \mathrm{~s} 2 \mathrm{p} 3$ & ${ }^{3} \mathrm{P} 0$ & ${ }^{3} \mathrm{D} 1$ & $3(1)$ & $5(2)$ & $5.865-2^{1}$ & $5.352-2$ & & \\
\hline $2 \mathrm{~s} 22 \mathrm{p} 2$ & $-2 \mathrm{~s} 2 \mathrm{p} 3$ & ${ }^{3} \mathrm{P} 0$ & ${ }^{3} \mathrm{D} 1$ & $5(1)$ & $3(2)$ & $1.487-4^{1}$ & $9.623-6$ & & \\
\hline $2 \mathrm{~s} 22 \mathrm{p} 2$ & $-2 \mathrm{~s} 2 \mathrm{p} 3$ & ${ }^{3} \mathrm{P} 0$ & ${ }^{3} \mathrm{D} 1$ & $5(1)$ & $5(2)$ & $3.399-3^{1}$ & $2.623-3$ & & \\
\hline $2 \mathrm{~s} 22 \mathrm{p} 2$ & $-2 \mathrm{~s} 2 \mathrm{p} 3$ & ${ }^{3} \mathrm{P} 0$ & ${ }^{3} \mathrm{D} 1$ & $5(1)$ & $7(1)$ & $5.037-2^{1}$ & $4.507-2$ & & \\
\hline $2 \mathrm{~s} 22 \mathrm{p} 2$ & $-2 \mathrm{~s} 2 \mathrm{p} 3$ & ${ }^{3} \mathrm{P} 0$ & ${ }^{3} \mathrm{D} 1$ & 9 & 15 & $5.33-2^{2}$ & $5.209-2$ & & \\
\hline $2 \mathrm{~s} 22 \mathrm{p} 2$ & $-2 \mathrm{~s} 2 \mathrm{p} 3$ & ${ }^{3} \mathrm{PO}$ & ${ }^{3} \mathrm{P} 1$ & $1(1)$ & $3(2)$ & $5.750-2^{1}$ & & & \\
\hline $2 \mathrm{~s} 22 \mathrm{p} 2$ & $-2 \mathrm{~s} 2 \mathrm{p} 3$ & ${ }^{3} \mathrm{P} 0$ & ${ }^{3} \mathrm{P} 1$ & $3(1)$ & $1(1)$ & $2.347-2^{1}$ & $2.071-2$ & & \\
\hline $2 \mathrm{~s} 22 \mathrm{p} 2$ & $-2 \mathrm{~s} 2 \mathrm{p} 3$ & ${ }^{3} \mathrm{P} 0$ & ${ }^{3} \mathrm{P} 1$ & $3(1)$ & $3(2)$ & $2.642-2^{1}$ & $1.574-2$ & & \\
\hline $2 \mathrm{~s} 22 \mathrm{p} 2$ & $-2 \mathrm{~s} 2 \mathrm{p} 3$ & ${ }^{3} \mathrm{P} 0$ & ${ }^{3} \mathrm{P} 1$ & $3(1)$ & $5(3)$ & $1.622-2^{1}$ & $1.318-2$ & & \\
\hline $2 \mathrm{~s} 22 \mathrm{p} 2$ & $-2 \mathrm{~s} 2 \mathrm{p} 3$ & ${ }^{3} \mathrm{P} 0$ & ${ }^{3} \mathrm{P} 1$ & $5(1)$ & $3(2)$ & $1.421-2^{1}$ & $1.947-2$ & & \\
\hline $2 \mathrm{~s} 22 \mathrm{p} 2$ & $-2 \mathrm{~s} 2 \mathrm{p} 3$ & ${ }^{3} \mathrm{P} 0$ & ${ }^{3} \mathrm{P} 1$ & $5(1)$ & $5(3)$ & $5.865-2^{1}$ & $5.271-2$ & & \\
\hline $2 \mathrm{~s} 22 \mathrm{p} 2$ & $-2 \mathrm{~s} 2 \mathrm{p} 3$ & ${ }^{3} \mathrm{P} 0$ & ${ }^{3} \mathrm{P} 1$ & 9 & 9 & $5.96-2^{2}$ & $6.096-2$ & & \\
\hline $2 \mathrm{~s} 22 \mathrm{p} 2$ & $-2 \mathrm{~s} 2 \mathrm{p} 3$ & ${ }^{3} \mathrm{P} 0$ & ${ }^{3} \mathrm{~S} 1$ & $1(1)$ & $3(3)$ & $7.053-2^{1}$ & $7.773-2$ & & \\
\hline $2 \mathrm{~s} 22 \mathrm{p} 2$ & $-2 \mathrm{~s} 2 \mathrm{p} 3$ & ${ }^{3} \mathrm{P} 0$ & ${ }^{3} \mathrm{~S} 1$ & $3(1)$ & $3(3)$ & $7.163-2^{1}$ & $7.544-2$ & & \\
\hline $2 \mathrm{~s} 22 \mathrm{p} 2$ & $-2 \mathrm{~s} 2 \mathrm{p} 3$ & ${ }^{3} \mathrm{P} 0$ & ${ }^{3} \mathrm{~S} 1$ & $5(1)$ & $3(3)$ & $8.013-2^{1}$ & $6.635-2$ & & \\
\hline $2 \mathrm{~s} 22 \mathrm{p} 2$ & $-2 \mathrm{~s} 2 \mathrm{p} 3$ & ${ }^{3} \mathrm{P} 0$ & ${ }^{3} \mathrm{~S} 1$ & 9 & 3 & $7.0-2^{2}$ & $7.060-2$ & & \\
\hline $2 \mathrm{~s} 22 \mathrm{p} 2$ & $-2 \mathrm{~s} 2 \mathrm{p} 3$ & ${ }^{3} \mathrm{P} 0$ & ${ }^{1} \mathrm{P} 1$ & $1(1)$ & $3(4)$ & $7.251-6^{1}$ & $8.405-3$ & & \\
\hline $2 \mathrm{~s} 22 \mathrm{p} 2$ & $-2 \mathrm{~s} 2 \mathrm{p} 3$ & ${ }^{3} \mathrm{P} 0$ & ${ }^{1} \mathrm{P} 1$ & $3(1)$ & $3(4)$ & $1.690-3^{1}$ & $3.256-3$ & & \\
\hline $2 \mathrm{~s} 22 \mathrm{p} 2$ & $-2 \mathrm{~s} 2 \mathrm{p} 3$ & ${ }^{3} \mathrm{P} 0$ & ${ }^{1} \mathrm{P} 1$ & $5(1)$ & $3(4)$ & $6.231-6^{1}$ & $5.59 \mathrm{E}-6$ & & \\
\hline $2 \mathrm{~s} 22 \mathrm{p} 2$ & $-2 \mathrm{~S} 2 \mathrm{p} 3$ & ${ }^{1} \mathrm{~S} 0$ & ${ }^{3} \mathrm{D} 1$ & $1(2)$ & $3(1)$ & $3.263-4^{1}$ & $2.257-4$ & & \\
\hline $2 \mathrm{~s} 22 \mathrm{p} 2$ & $-2 \mathrm{~s} 2 \mathrm{p} 3$ & ${ }^{1} \mathrm{~S} 0$ & ${ }^{3} \mathrm{P} 1$ & $1(2)$ & $3(2)$ & $7.137-4^{1}$ & 4.438-4 & & \\
\hline $2 \mathrm{~s} 22 \mathrm{p} 2$ & $-2 \mathrm{~s} 2 \mathrm{p} 3$ & ${ }^{1} \mathrm{~S} 0$ & ${ }^{3} \mathrm{~S} 1$ & $1(2)$ & $3(3)$ & $9.600-4^{1}$ & $8.290-3$ & & \\
\hline $2 \mathrm{~s} 22 \mathrm{p} 2$ & $-2 \mathrm{~s} 2 \mathrm{p} 3$ & ${ }^{1} \mathrm{~S} 0$ & ${ }^{1} \mathrm{P} 1$ & $1(2)$ & $3(4)$ & $1.514-1^{1}, 1.35-1^{2}$ & $1.314-1$ & & \\
\hline $2 \mathrm{~s} 22 \mathrm{p} 2$ & $-2 \mathrm{~s} 22 \mathrm{p} 3 \mathrm{~s}$ & ${ }^{3} \mathrm{P} 0$ & ${ }^{3} \mathrm{P} 1$ & $1(1)$ & $3(5)$ & $4.966-2^{1}$ & $5.665-2$ & & \\
\hline $2 \mathrm{~s} 22 \mathrm{p} 2$ & $-2 \mathrm{~s} 22 \mathrm{p} 3 \mathrm{~s}$ & ${ }^{3} \mathrm{P} 0$ & ${ }^{3} \mathrm{P} 1$ & $3(1)$ & $1(2)$ & $1.734-2^{1}$ & $1.874-2$ & & \\
\hline $2 \mathrm{~s} 22 \mathrm{p} 2$ & $-2 \mathrm{~s} 22 \mathrm{p} 3 \mathrm{~s}$ & ${ }^{3} \mathrm{P} 0$ & ${ }^{3} \mathrm{P} 1$ & $3(1)$ & $3(5)$ & $1.157-2^{1}$ & $1.431-2$ & & \\
\hline $2 \mathrm{~s} 22 \mathrm{p} 2$ & $-2 \mathrm{~s} 22 \mathrm{p} 3 \mathrm{~s}$ & ${ }^{3} \mathrm{P} 0$ & ${ }^{3} \mathrm{P} 1$ & $3(1)$ & $5(5)$ & $2.194-2^{1}$ & $2.357-2$ & & \\
\hline $2 \mathrm{~s} 22 \mathrm{p} 2$ & $-2 \mathrm{~s} 22 \mathrm{p} 3 \mathrm{~s}$ & ${ }^{3} \mathrm{P} 0$ & ${ }^{3} \mathrm{P} 1$ & $5(1)$ & $3(5)$ & $1.388-2^{1}$ & $1.110-2$ & & \\
\hline $2 \mathrm{~s} 22 \mathrm{p} 2$ & $-2 \mathrm{~s} 22 \mathrm{p} 3 \mathrm{~s}$ & ${ }^{3} \mathrm{P} 0$ & ${ }^{3} \mathrm{P} 1$ & $5(1)$ & $5(5)$ & $3.843-2^{1}$ & $4.274-2$ & & \\
\hline $2 \mathrm{~s} 22 \mathrm{p} 2$ & $-2 \mathrm{~s} 22 \mathrm{p} 3 \mathrm{~s}$ & ${ }^{3} \mathrm{P} 0$ & ${ }^{3} \mathrm{P} 1$ & 9 & 9 & $6.07-2^{2}$ & $5.507-2$ & & \\
\hline 2S2p3 & $-2 \mathrm{p} 4$ & ${ }^{3} \mathrm{D} 1$ & ${ }^{3} \mathrm{P} 0$ & $3(1)$ & $1(3)$ & $3.817-2^{1}$ & $3.321-2$ & & \\
\hline 2S2p3 & $-2 \mathrm{p} 4$ & ${ }^{3} \mathrm{D} 1$ & ${ }^{3} \mathrm{P} 0$ & $3(1)$ & $3(2)$ & $3.765-2^{1}$ & $3.720-2$ & & \\
\hline 2S2p3 & $-2 \mathrm{p} 4$ & ${ }^{3} \mathrm{D} 1$ & ${ }^{3} \mathrm{P} 0$ & $3(1)$ & $5(3)$ & $4.857-3^{1}$ & $3.430-3$ & & \\
\hline 2S2p3 & $-2 \mathrm{p} 4$ & ${ }^{3} \mathrm{D} 1$ & ${ }^{3} \mathrm{P} 0$ & 15 & 9 & $6.867-2^{2}$ & $1.452-2$ & & \\
\hline $2 \mathrm{~s} 2 \mathrm{p} 3$ & $-2 \mathrm{p} 4$ & ${ }^{3} \mathrm{~S} 1$ & ${ }^{3} \mathrm{P} 0$ & $3(3)$ & $1(3)$ & $7.175-2^{1}$ & $1.657-2$ & & \\
\hline $2 \mathrm{~s} 2 \mathrm{p} 3$ & $-2 \mathrm{p} 4$ & ${ }^{3} \mathrm{~S} 1$ & ${ }^{3} \mathrm{P} 0$ & $3(3)$ & $3(2)$ & $6.660-2^{1}$ & $5.504-2$ & & \\
\hline $2 \mathrm{~s} 2 \mathrm{p} 3$ & $-2 \mathrm{p} 4$ & ${ }^{3} \mathrm{~S} 1$ & ${ }^{3} \mathrm{P} 0$ & $3(3)$ & $5(3)$ & $5.916-2^{1}$ & $9.966-2$ & & \\
\hline $2 \mathrm{~s} 2 \mathrm{p} 3$ & $-2 \mathrm{p} 4$ & ${ }^{3} \mathrm{~S} 1$ & ${ }^{3} \mathrm{P} 0$ & 3 & 9 & $1.58-1^{2}$ & $1.716-1$ & & \\
\hline $2 \mathrm{~s} 2 \mathrm{p} 3$ & $-2 \mathrm{p} 4$ & ${ }^{1} \mathrm{P} 1$ & ${ }^{3} \mathrm{P} 0$ & $3(4)$ & $1(3)$ & $8.069-5^{1}$ & $1.568-3$ & & \\
\hline $2 \mathrm{~s} 2 \mathrm{p} 3$ & $-2 \mathrm{p} 4$ & ${ }^{1} \mathrm{P} 1$ & ${ }^{3} \mathrm{P} 0$ & $3(4)$ & $3(2)$ & $1.103-3^{1}$ & $1.809-3$ & & \\
\hline $2 \mathrm{~s} 2 \mathrm{p} 3$ & $-2 \mathrm{p} 4$ & ${ }^{1} \mathrm{P} 1$ & ${ }^{3} \mathrm{P} 0$ & $3(4)$ & $5(3)$ & $-3.784-4^{1}$ & $4.007-4$ & & \\
\hline $2 \mathrm{~s} 22 \mathrm{p} 2$ & $-2 \mathrm{~s} 2 \mathrm{p} 3$ & ${ }^{3} \mathrm{P} 0$ & ${ }^{5} \mathrm{~S} 1$ & $3(1)$ & $5(1)$ & & $3.752-5$ & $7.28+5^{3}$ & $7.01+5$ \\
\hline $2 \mathrm{~s} 22 \mathrm{p} 2$ & $-2 \mathrm{~s} 2 \mathrm{p} 3$ & ${ }^{3} \mathrm{PO}$ & ${ }^{5} \mathrm{~S} 1$ & $5(1)$ & $5(1)$ & & $4.810-5$ & $1.35+6^{3}$ & $1.34+6$ \\
\hline $2 \mathrm{~s} 22 \mathrm{p} 2$ & $-2 \mathrm{~s} 2 \mathrm{p} 3$ & ${ }^{1} \mathrm{D} 0$ & ${ }^{5} \mathrm{~S} 1$ & $5(2)$ & $5(1)$ & & $4.730-7$ & $7.15+3^{3}$ & $6.26+3$ \\
\hline
\end{tabular}

1 Zhang \& Sampson (1997), ${ }^{2}$ Luo \& Pradhan (1989), ${ }^{3}$ Mendoza et al. (1999). 
Table 8. a. Sample set of reprocessed $f$ - and $A$-values for Fe XXI using observed transition energies. The negative sign for the energies is omitted for convenience. $I_{i}$ and $I_{j}$ are the level indices

\begin{tabular}{|c|c|c|c|c|c|c|c|c|c|}
\hline$C_{i}$ & $C_{j}$ & $S_{i} L_{i} \pi_{i}$ & $S_{j} L_{j} \pi_{j}$ & $g_{i}\left(I_{i}\right)$ & $g_{j}\left(I_{j}\right)$ & $E_{i}(\mathrm{Ry})$ & $E_{j}(\mathrm{Ry})$ & $f$ & $A_{j i}\left(\mathrm{~s}^{-1}\right)$ \\
\hline $2 \mathrm{~s}^{2} 2 \mathrm{p} 2$ & $-2 \mathrm{~s} 2 \mathrm{p}^{3}$ & ${ }^{3} \mathrm{P}^{\mathrm{e}}$ & ${ }^{3} \mathrm{D}^{\circ}$ & $1(1)$ & $3(1)$ & 124.1100 & 117.0400 & $8.491 \mathrm{E}-02$ & $1.14 \mathrm{E}+10$ \\
\hline $2 \mathrm{~s}^{2} 2 \mathrm{p}^{2}$ & $-2 \mathrm{~s} 2 \mathrm{p}^{3}$ & ${ }^{3} \mathrm{P}^{\mathrm{e}}$ & ${ }^{3} \mathrm{P}^{\mathrm{o}}$ & $1(1)$ & $3(2)$ & 124.1100 & 115.6900 & $2.252 \mathrm{E}-02$ & $4.27 \mathrm{E}+09$ \\
\hline $2 \mathrm{~s}^{2} 2 \mathrm{p}^{2}$ & $-2 \mathrm{~s} 2 \mathrm{p}^{3}$ & ${ }^{3} \mathrm{P}^{\mathrm{e}}$ & ${ }^{3} \mathrm{~S}^{\circ}$ & $1(1)$ & $3(3)$ & 124.1100 & 114.1300 & $3.579 \mathrm{E}-02$ & $9.55 \mathrm{E}+09$ \\
\hline $2 s^{2} 2 p^{2}$ & $-2 \mathrm{~s} 2 \mathrm{p}^{3}$ & ${ }^{3} \mathrm{P}^{\mathrm{e}}$ & ${ }^{1} \mathrm{P}^{\mathrm{o}}$ & $1(1)$ & $3(4)$ & 124.1100 & 112.6200 & $1.028 \mathrm{E}-04$ & $3.63 \mathrm{E}+07$ \\
\hline $2 \mathrm{~s}^{2} 2 \mathrm{p}^{2}$ & $-2 \mathrm{~s}^{2} 2 \mathrm{p} 3 \mathrm{~d}$ & ${ }^{3} \mathrm{P}^{\mathrm{e}}$ & ${ }^{1} \mathrm{P}^{\mathrm{o}}$ & $1(1)$ & $3(11)$ & 124.1100 & 48.5350 & $2.771 \mathrm{E}-02$ & $4.24 \mathrm{E}+11$ \\
\hline $2 \mathrm{~s}^{2} 2 \mathrm{p}^{2}$ & $-2 \mathrm{~s}^{2} 2 \mathrm{p} 4 \mathrm{~d}$ & ${ }^{3} \mathrm{P}^{\mathrm{e}}$ & ${ }^{3} \mathrm{D}^{\circ}$ & $1(1)$ & 3 (39) & 124.1100 & 27.6930 & $1.027 \mathrm{E}-01$ & $2.56 \mathrm{E}+12$ \\
\hline $2 \mathrm{~s}^{2} 2 \mathrm{p}^{2}$ & $-2 s^{2} 2 p 4 d$ & ${ }^{3} \mathrm{P}^{\mathrm{e}}$ & ${ }^{3} \mathrm{P}^{\circ}$ & $1(1)$ & $3(40)$ & 124.1100 & 26.7180 & $1.376 \mathrm{E}-01$ & $3.49 \mathrm{E}+12$ \\
\hline $2 \mathrm{~s}^{2} 2 \mathrm{p}^{2}$ & $-2 \mathrm{~s}^{2} 2 \mathrm{p} 5 \mathrm{~d}$ & ${ }^{3} \mathrm{P}^{\mathrm{e}}$ & ${ }^{3} \mathrm{P}^{\mathrm{o}}$ & $1(1)$ & $3(67)$ & 124.1100 & 16.4940 & $5.546 \mathrm{E}-02$ & $1.72 \mathrm{E}+12$ \\
\hline $2 s^{2} 2 p^{2}$ & $-2 \mathrm{~s} 2 \mathrm{p}^{3}$ & ${ }^{1} \mathrm{~S}^{\mathrm{e}}$ & ${ }^{3} \mathrm{D}^{\mathrm{o}}$ & $1(2)$ & $3(1)$ & 120.7300 & 117.0400 & $1.066 \mathrm{E}-03$ & $3.88 \mathrm{E}+07$ \\
\hline $2 s^{2} 2 p^{2}$ & $-2 \mathrm{~s} 2 \mathrm{p}^{3}$ & ${ }^{1} \mathrm{~S}^{\mathrm{e}}$ & ${ }^{3} \mathrm{P}^{\mathrm{o}}$ & $1(2)$ & $3(2)$ & 120.7300 & 115.6900 & $2.208 \mathrm{E}-03$ & $1.50 \mathrm{E}+08$ \\
\hline $2 \mathrm{~s}^{2} 2 \mathrm{p}^{2}$ & $-2 \mathrm{~s} 2 \mathrm{p}^{3}$ & ${ }^{1} \mathrm{~S}^{\mathrm{e}}$ & ${ }^{3} \mathrm{~S}^{\mathrm{o}}$ & $1(2)$ & $3(3)$ & 120.7300 & 114.1300 & $5.568 \mathrm{E}-03$ & $6.49 \mathrm{E}+08$ \\
\hline $2 \mathrm{~s}^{2} 2 \mathrm{p}^{2}$ & $-2 \mathrm{~s} 2 \mathrm{p}^{3}$ & ${ }^{1} \mathrm{~S}^{\mathrm{e}}$ & ${ }^{1} \mathrm{P}^{\mathrm{o}}$ & $1(2)$ & $3(4)$ & 120.7300 & 112.6200 & $9.789 \mathrm{E}-02$ & $1.72 \mathrm{E}+10$ \\
\hline $2 s^{2} 2 p^{2}$ & $-2 \mathrm{~s}^{2} 2 \mathrm{p} 3 \mathrm{~d}$ & ${ }^{1} \mathrm{~S}^{\mathrm{e}}$ & ${ }^{1} \mathrm{P}^{\mathrm{o}}$ & $1(2)$ & 3 (11) & 120.7300 & 48.5350 & $1.276 \mathrm{E}+00$ & $1.78 \mathrm{E}+13$ \\
\hline $2 \mathrm{~s}^{2} 2 \mathrm{p}^{2}$ & $-2 s^{2} 2 p 4 d$ & ${ }^{1} \mathrm{~S}^{\mathrm{e}}$ & ${ }^{3} \mathrm{D}^{\mathrm{o}}$ & $1(2)$ & $3(39)$ & 120.7300 & 27.6930 & $2.722 \mathrm{E}-02$ & $6.31 \mathrm{E}+11$ \\
\hline $2 \mathrm{~s}^{2} 2 \mathrm{p}^{2}$ & $-2 \mathrm{~s}^{2} 2 \mathrm{p} 4 \mathrm{~d}$ & ${ }^{1} \mathrm{~S}^{\mathrm{e}}$ & ${ }^{3} \mathrm{P}^{\mathrm{o}}$ & $1(2)$ & $3(40)$ & 120.7300 & 26.7180 & $1.458 \mathrm{E}-03$ & $3.45 \mathrm{E}+10$ \\
\hline $2 s^{2} 2 p^{2}$ & $-2 \mathrm{~s}^{2} 2 \mathrm{p} 5 \mathrm{~d}$ & ${ }^{1} \mathrm{~S}^{\mathrm{e}}$ & ${ }^{3} \mathrm{P}^{\mathrm{o}}$ & $1(2)$ & 3 (67) & 120.7300 & 16.4940 & $1.678 \mathrm{E}-03$ & $4.88 \mathrm{E}+10$ \\
\hline $2 \mathrm{~s} 2 \mathrm{p}^{3}$ & $-2 p^{4}$ & ${ }^{3} \mathrm{D}^{\mathrm{o}}$ & ${ }^{3} \mathrm{P}^{\mathrm{e}}$ & $3(1)$ & $1(3)$ & 117.0400 & 108.3000 & $1.911 \mathrm{E}-02$ & $3.52 \mathrm{E}+10$ \\
\hline $2 \mathrm{~s} 2 \mathrm{p}^{3}$ & $-2 p^{4}$ & ${ }^{3} \mathrm{P}^{\mathrm{o}}$ & ${ }^{3} \mathrm{P}^{\mathrm{e}}$ & $3(2)$ & $1(3)$ & 115.6900 & 108.3000 & $1.379 \mathrm{E}-02$ & $1.81 \mathrm{E}+10$ \\
\hline $2 \mathrm{~s} 2 \mathrm{p}^{3}$ & $-2 p^{4}$ & ${ }^{3} \mathrm{~S}^{\circ}$ & ${ }^{3} \mathrm{P}^{\mathrm{e}}$ & $3(3)$ & $1(3)$ & 114.1300 & 108.3000 & $2.095 \mathrm{E}-02$ & $1.72 \mathrm{E}+10$ \\
\hline $2 \mathrm{~s} 2 \mathrm{p}^{3}$ & $-2 p^{4}$ & ${ }^{1} \mathrm{P}^{\mathrm{o}}$ & ${ }^{3} \mathrm{P}^{\mathrm{e}}$ & $3(4)$ & $1(3)$ & 112.6200 & 108.3000 & $3.176 \mathrm{E}-05$ & $1.43 \mathrm{E}+07$ \\
\hline $2 p^{4}$ & $-2 \mathrm{~s}^{2} 2 \mathrm{p} 3 \mathrm{~d}$ & ${ }^{3} \mathrm{P}^{\mathrm{e}}$ & ${ }^{1} \mathrm{P}^{\mathrm{o}}$ & $1(3)$ & 3 (11) & 108.3000 & 48.5350 & $1.067 \mathrm{E}-06$ & $1.02 \mathrm{E}+07$ \\
\hline $2 p^{4}$ & $-2 \mathrm{~s}^{2} 2 \mathrm{p} 4 \mathrm{~d}$ & ${ }^{3} \mathrm{P}^{\mathrm{e}}$ & ${ }^{3} \mathrm{D}^{\circ}$ & $1(3)$ & $3(39)$ & 108.3000 & 27.6930 & $2.334 \mathrm{E}-05$ & $4.06 \mathrm{E}+08$ \\
\hline $2 p^{4}$ & $-2 s^{2} 2 p 4 d$ & ${ }^{3} \mathrm{P}^{\mathrm{e}}$ & ${ }^{3} \mathrm{P}^{\mathrm{o}}$ & $1(3)$ & $3(40)$ & 108.3000 & 26.7180 & $1.608 \mathrm{E}-04$ & $2.87 \mathrm{E}+09$ \\
\hline $2 p^{4}$ & $-2 \mathrm{~s}^{2} 2 \mathrm{p} 5 \mathrm{~d}$ & ${ }^{3} \mathrm{P}^{\mathrm{e}}$ & ${ }^{3} \mathrm{P}^{\mathrm{o}}$ & $1(3)$ & 3 (67) & 108.3000 & 16.4940 & $5.095 \mathrm{E}-04$ & $1.15 \mathrm{E}+10$ \\
\hline $2 \mathrm{~s} 2 \mathrm{p}^{3}$ & $-2 p^{4}$ & ${ }^{3} \mathrm{D}^{\mathrm{o}}$ & ${ }^{1} \mathrm{~S}^{\mathrm{e}}$ & $3(1)$ & $1(4)$ & 117.0400 & 105.4500 & $8.736 \mathrm{E}-05$ & $2.83 \mathrm{E}+08$ \\
\hline $2 \mathrm{~s} 2 \mathrm{p}^{3}$ & $-2 p^{4}$ & ${ }^{3} \mathrm{P}^{\mathrm{o}}$ & ${ }^{1} \mathrm{~S}^{\mathrm{e}}$ & $3(2)$ & $1(4)$ & 115.6900 & 105.4500 & $1.739 \mathrm{E}-03$ & $4.39 \mathrm{E}+09$ \\
\hline $2 \mathrm{~s} 2 \mathrm{p}^{3}$ & $-2 p^{4}$ & ${ }^{3} \mathrm{~S}^{\circ}$ & ${ }^{1} \mathrm{~S}^{\mathrm{e}}$ & $3(3)$ & $1(4)$ & 114.1300 & 105.4500 & $2.770 \mathrm{E}-03$ & $5.03 \mathrm{E}+09$ \\
\hline $2 \mathrm{~s} 2 \mathrm{p}^{3}$ & $-2 p^{4}$ & ${ }^{1} \mathrm{P}^{\mathrm{o}}$ & ${ }^{1} \mathrm{~S}^{\mathrm{e}}$ & $3(4)$ & $1(4)$ & 112.6200 & 105.4500 & $6.055 \mathrm{E}-02$ & $7.50 \mathrm{E}+10$ \\
\hline $2 p^{4}$ & $-2 \mathrm{~s}^{2} 2 \mathrm{p} 3 \mathrm{~d}$ & ${ }^{1} \mathrm{~S}^{\mathrm{e}}$ & ${ }^{1} \mathrm{P}^{\mathrm{o}}$ & $1(4)$ & 3 (11) & 105.4500 & 48.5350 & $8.194 \mathrm{E}-05$ & $7.11 \mathrm{E}+08$ \\
\hline $2 p^{4}$ & $-2 \mathrm{~s}^{2} 2 \mathrm{p} 4 \mathrm{~d}$ & ${ }^{1} \mathrm{~S}^{\mathrm{e}}$ & ${ }^{3} \mathrm{D}^{\circ}$ & $1(4)$ & $3(39)$ & 105.4500 & 27.6930 & $7.892 \mathrm{E}-06$ & $1.28 \mathrm{E}+08$ \\
\hline $2 p^{4}$ & $-2 \mathrm{~s}^{2} 2 \mathrm{p} 4 \mathrm{~d}$ & ${ }^{1} \mathrm{~S}^{\mathrm{e}}$ & ${ }^{3} \mathrm{P}^{\mathrm{o}}$ & $1(4)$ & $3(40)$ & 105.4500 & 26.7180 & $1.302 \mathrm{E}-05$ & $2.16 \mathrm{E}+08$ \\
\hline $2 p^{4}$ & $-2 \mathrm{~s}^{2} 2 \mathrm{p} 5 \mathrm{~d}$ & ${ }^{1} \mathrm{~S}^{\mathrm{e}}$ & ${ }^{3} \mathrm{P}^{\mathrm{o}}$ & $1(4)$ & $3(67)$ & 105.4500 & 16.4940 & $3.718 \mathrm{E}-08$ & $7.88 \mathrm{E}+05$ \\
\hline $2 \mathrm{~s}^{2} 2 \mathrm{p}^{2}$ & $-2 \mathrm{~s} 2 \mathrm{p}^{3}$ & ${ }^{3} \mathrm{P}^{\mathrm{e}}$ & ${ }^{3} \mathrm{P}^{\mathrm{o}}$ & $3(1)$ & 1 (1) & 123.4400 & 115.7600 & $1.537 \mathrm{E}-02$ & $2.18 \mathrm{E}+10$ \\
\hline $2 \mathrm{~s} 2 \mathrm{p}^{3}$ & $-2 p^{4}$ & ${ }^{3} \mathrm{P}^{\circ}$ & ${ }^{3} \mathrm{P}^{\mathrm{e}}$ & $1(1)$ & $3(2)$ & 115.7600 & 108.2500 & $3.117 \mathrm{E}-02$ & $4.71 \mathrm{E}+09$ \\
\hline $2 \mathrm{~s}^{2} 2 \mathrm{p}^{2}$ & $-2 \mathrm{~s} 2 \mathrm{p}^{3}$ & ${ }^{3} \mathrm{P}^{\mathrm{e}}$ & ${ }^{3} \mathrm{D}^{\circ}$ & $3(1)$ & $3(1)$ & 123.4400 & 117.0400 & $2.453 \mathrm{E}-03$ & $8.07 \mathrm{E}+08$ \\
\hline $2 \mathrm{~s}^{2} 2 \mathrm{p}^{2}$ & $-2 \mathrm{~s} 2 \mathrm{p}^{3}$ & ${ }^{3} \mathrm{P}^{\mathrm{e}}$ & ${ }^{3} \mathrm{P}^{\mathrm{o}}$ & $3(1)$ & $3(2)$ & 123.4400 & 115.6900 & $3.234 \mathrm{E}-02$ & $1.56 \mathrm{E}+10$ \\
\hline $2 \mathrm{~s}^{2} 2 \mathrm{p}^{2}$ & $-2 \mathrm{~s} 2 \mathrm{p}^{3}$ & ${ }^{3} \mathrm{P}^{\mathrm{e}}$ & ${ }^{3} \mathrm{~S}^{\mathrm{o}}$ & $3(1)$ & $3(3)$ & 123.4400 & 114.1300 & 3.671E-02 & $2.56 \mathrm{E}+10$ \\
\hline $2 \mathrm{~s}^{2} 2 \mathrm{p}^{2}$ & $-2 \mathrm{~s} 2 \mathrm{p}^{3}$ & ${ }^{3} \mathrm{P}^{\mathrm{e}}$ & ${ }^{1} \mathrm{P}^{\mathrm{o}}$ & $3(1)$ & $3(4)$ & 123.4400 & 112.6200 & $5.291 \mathrm{E}-03$ & $4.98 \mathrm{E}+09$ \\
\hline $2 s^{2} 2 p^{2}$ & $-2 \mathrm{~s}^{2} 2 \mathrm{p} 3 \mathrm{~d}$ & ${ }^{3} \mathrm{P}^{\mathrm{e}}$ & ${ }^{1} \mathrm{P}^{\mathrm{o}}$ & $3(1)$ & 3 (11) & 123.4400 & 48.5350 & $6.550 \mathrm{E}-03$ & $2.95 \mathrm{E}+11$ \\
\hline $2 \mathrm{~s}^{2} 2 \mathrm{p}^{2}$ & $-2 \mathrm{~s}^{2} 2 \mathrm{p} 4 \mathrm{~d}$ & ${ }^{3} \mathrm{P}^{\mathrm{e}}$ & ${ }^{3} \mathrm{D}^{\circ}$ & $3(1)$ & $3(39)$ & 123.4400 & 27.6930 & $4.825 \mathrm{E}-02$ & $3.55 \mathrm{E}+12$ \\
\hline $2 s^{2} 2 p^{2}$ & $-2 \mathrm{~s}^{2} 2 \mathrm{p} 4 \mathrm{~d}$ & ${ }^{3} \mathrm{P}^{\mathrm{e}}$ & ${ }^{3} \mathrm{P}^{\mathrm{o}}$ & $3(1)$ & $3(40)$ & 123.4400 & 26.7180 & $2.762 \mathrm{E}-03$ & $2.08 \mathrm{E}+11$ \\
\hline $2 s^{2} 2 p^{2}$ & $-2 \mathrm{~s}^{2} 2 \mathrm{p} 5 \mathrm{~d}$ & ${ }^{3} \mathrm{P}^{\mathrm{e}}$ & ${ }^{3} \mathrm{P}^{\circ}$ & $3(1)$ & $3(67)$ & 123.4400 & 16.4940 & $4.713 \mathrm{E}-04$ & $4.33 \mathrm{E}+10$ \\
\hline $2 \mathrm{~s} 2 \mathrm{p}^{3}$ & $-2 p^{4}$ & ${ }^{3} \mathrm{D}^{0}$ & ${ }^{3} \mathrm{P}^{\mathrm{e}}$ & $3(1)$ & $3(2)$ & 117.0400 & 108.2500 & $2.322 \mathrm{E}-02$ & $1.44 \mathrm{E}+10$ \\
\hline $2 \mathrm{~s} 2 \mathrm{p}^{3}$ & $-2 p^{4}$ & ${ }^{3} \mathrm{P}^{\mathrm{o}}$ & ${ }^{3} \mathrm{P}^{\mathrm{e}}$ & $3(2)$ & $3(2)$ & 115.6900 & 108.2500 & $1.921 \mathrm{E}-04$ & $8.54 \mathrm{E}+07$ \\
\hline $2 \mathrm{~s} 2 \mathrm{p}^{3}$ & $-2 p^{4}$ & ${ }^{3} \mathrm{~S}^{\mathrm{o}}$ & ${ }^{3} \mathrm{P}^{\mathrm{e}}$ & $3(3)$ & $3(2)$ & 114.1300 & 108.2500 & $4.638 \mathrm{E}-02$ & $1.29 \mathrm{E}+10$ \\
\hline $2 \mathrm{~s} 2 \mathrm{p}^{3}$ & $-2 p^{4}$ & ${ }^{1} \mathrm{P}^{\mathrm{o}}$ & ${ }^{3} \mathrm{P}^{\mathrm{e}}$ & $3(4)$ & $3(2)$ & 112.6200 & 108.2500 & $4.176 \mathrm{E}-03$ & $6.41 \mathrm{E}+08$ \\
\hline $2 p^{4}$ & $-2 s^{2} 2 p 3 d$ & ${ }^{3} \mathrm{P}^{\mathrm{e}}$ & ${ }^{1} \mathrm{P}^{\mathrm{o}}$ & $3(2)$ & $3(11)$ & 108.2500 & 48.5350 & $5.993 \mathrm{E}-06$ & $1.72 \mathrm{E}+08$ \\
\hline $2 p^{4}$ & $-2 \mathrm{~s}^{2} .2 \mathrm{p} .4 \mathrm{~d}$ & ${ }^{3} \mathrm{P}^{\mathrm{e}}$ & ${ }^{3} \mathrm{D}^{\circ}$ & $3(2)$ & $3(39)$ & 108.2500 & 27.6930 & $5.489 \mathrm{E}-06$ & $2.86 \mathrm{E}+08$ \\
\hline $2 p^{4}$ & $-2 \mathrm{~s}^{2} .2 \mathrm{p} .4 \mathrm{~d}$ & ${ }^{3} \mathrm{P}^{\mathrm{e}}$ & ${ }^{3} \mathrm{P}^{\mathrm{o}}$ & $3(2)$ & $3(40)$ & 108.2500 & 26.7180 & $2.160 \mathrm{E}-06$ & $1.15 \mathrm{E}+08$ \\
\hline $2 p^{4}$ & $-2 \mathrm{~s}^{2} .2 \mathrm{p} .5 \mathrm{~d}$ & ${ }^{3} \mathrm{P}^{\mathrm{e}}$ & ${ }^{3} \mathrm{P}^{\mathrm{o}}$ & $3(2)$ & $3(67)$ & 108.2500 & 16.4940 & $1.847 \mathrm{E}-04$ & $1.25 \mathrm{E}+10$ \\
\hline $2 \mathrm{~s}^{2} \cdot 2 \mathrm{p} 2$ & $-2 \mathrm{~s} \cdot 2 \mathrm{p} 3$ & ${ }^{3} \mathrm{P}^{\mathrm{e}}$ & ${ }^{5} \mathrm{~S}^{\circ}$ & $3(1)$ & $5(1)$ & 123.4400 & 119.6800 & $5.155 \mathrm{E}-04$ & $3.51 \mathrm{E}+07$ \\
\hline $2 \mathrm{~s}^{2} .2 \mathrm{p} 2$ & $-2 \mathrm{~s} \cdot 2 \mathrm{p} 3$ & ${ }^{3} \mathrm{P}^{\mathrm{e}}$ & ${ }^{3} \mathrm{D}^{\circ}$ & $3(1)$ & $5(2)$ & 123.4400 & 117.0300 & $4.621 \mathrm{E}-02$ & $9.15 \mathrm{E}+09$ \\
\hline $2 \mathrm{~s}^{2} .2 \mathrm{p} 2$ & $-2 \mathrm{~s} \cdot 2 \mathrm{p} 3$ & ${ }^{3} \mathrm{P}^{\mathrm{e}}$ & ${ }^{3} \mathrm{P}^{\circ}$ & $3(1)$ & $5(3)$ & 123.4400 & 115.5300 & $1.379 \mathrm{E}-03$ & $4.16 \mathrm{E}+08$ \\
\hline $2 \mathrm{~s}^{2} .2 \mathrm{p} 2$ & $-2 \mathrm{~s} 2 \mathrm{p} 3$ & ${ }^{3} \mathrm{P}^{\mathrm{e}}$ & ${ }^{1} \mathrm{D}^{\circ}$ & $3(1)$ & $5(4)$ & 123.4400 & 113.8500 & $9.379 \mathrm{E}-04$ & $4.16 \mathrm{E}+08$ \\
\hline $2 \mathrm{~s}^{2} .2 \mathrm{p} 2$ & $-2 \mathrm{~s}^{2} .2 \mathrm{p} .3 \mathrm{~d}$ & ${ }^{3} \mathrm{P}^{\mathrm{e}}$ & ${ }^{1} \mathrm{D}^{\mathrm{o}}$ & $3(1)$ & $5(6)$ & 123.4400 & 50.3200 & $4.192 \mathrm{E}-05$ & $1.08 \mathrm{E}+09$ \\
\hline $2 \mathrm{~s}^{2} .2 \mathrm{p} 2$ & $-2 \mathrm{~s}^{2} .2 \mathrm{p} .3 \mathrm{~d}$ & ${ }^{3} \mathrm{P}^{\mathrm{e}}$ & ${ }^{3} \mathrm{D}^{\circ}$ & $3(1)$ & $5(8)$ & 123.4400 & 49.5060 & $8.502 \mathrm{E}-01$ & $2.24 \mathrm{E}+13$ \\
\hline $2 \mathrm{~s}^{2} .2 \mathrm{p} 2$ & $-2 \mathrm{~s}^{2} .2 \mathrm{p} .3 \mathrm{~d}$ & ${ }^{3} \mathrm{P}^{\mathrm{e}}$ & ${ }^{3} \mathrm{P}^{\mathrm{o}}$ & $3(1)$ & $5(9)$ & 123.4400 & 49.1090 & $6.579 \mathrm{E}-03$ & $1.75 \mathrm{E}+11$ \\
\hline $2 \mathrm{~s}^{2} .2 \mathrm{p} 2$ & $-2 \mathrm{~s}^{2} .2 \mathrm{p} .4 \mathrm{~d}$ & ${ }^{3} \mathrm{P}^{\mathrm{e}}$ & ${ }^{3} \mathrm{P}^{\mathrm{o}}$ & $3(1)$ & $5(39)$ & 123.4400 & 27.7030 & $1.682 \mathrm{E}-02$ & $7.43 \mathrm{E}+11$ \\
\hline $2 \mathrm{~s}^{2} \cdot 2 \mathrm{p} 2$ & $-2 \mathrm{~s}^{2} .2 \mathrm{p} .4 \mathrm{~d}$ & ${ }^{3} \mathrm{P}^{\mathrm{e}}$ & ${ }^{1} \mathrm{D}^{\circ}$ & $3(1)$ & $5(40)$ & 123.4400 & 26.8370 & $6.750 \mathrm{E}-02$ & $3.04 \mathrm{E}+12$ \\
\hline $2 \mathrm{~s}^{2} .2 \mathrm{p} 2$ & $-2 \mathrm{~s}^{2} \cdot 2 \mathrm{p} .4 \mathrm{~d}$ & ${ }^{3} \mathrm{P}^{\mathrm{e}}$ & ${ }^{3} \mathrm{D}^{\circ}$ & $3(1)$ & $5(41)$ & 123.4400 & 27.0190 & $7.369 \mathrm{E}-02$ & $3.30 \mathrm{E}+12$ \\
\hline $2 \mathrm{~s}^{2} .2 \mathrm{p} 2$ & $-2 \mathrm{~s}^{2} .2 \mathrm{p} .5 \mathrm{~d}$ & ${ }^{3} \mathrm{P}^{\mathrm{e}}$ & ${ }^{1} \mathrm{D}^{\mathrm{o}}$ & $3(1)$ & $5(72)$ & 123.4400 & 16.4940 & $1.839 \mathrm{E}-02$ & $1.01 \mathrm{E}+12$ \\
\hline $2 \mathrm{~s} \cdot 2 \mathrm{p} 3$ & $-2 p^{4}$ & ${ }^{5} \mathrm{~S}^{\circ}$ & ${ }^{3} \mathrm{P}^{\mathrm{e}}$ & $5(1)$ & $3(2)$ & 119.6800 & 108.2500 & $1.586 \mathrm{E}-04$ & $2.77 \mathrm{E}+08$ \\
\hline $2 \mathrm{~s} \cdot 2 \mathrm{p} 3$ & $-2 p^{4}$ & ${ }^{3} \mathrm{D}^{\circ}$ & ${ }^{3} \mathrm{P}^{\mathrm{e}}$ & $5(2)$ & $3(2)$ & 117.0300 & 108.2500 & $2.093 \mathrm{E}-02$ & $2.16 \mathrm{E}+10$ \\
\hline $2 \mathrm{~s} \cdot 2 \mathrm{p} 3$ & $-2 p^{4}$ & ${ }^{3} \mathrm{P}^{\mathrm{o}}$ & ${ }^{3} \mathrm{P}^{\mathrm{e}}$ & $5(3)$ & $3(2)$ & 115.5300 & 108.2500 & $2.219 \mathrm{E}-02$ & $1.57 \mathrm{E}+10$ \\
\hline
\end{tabular}


Table 8. b. Fine structure transitions of Ar XIII are ordered in $L S$ multiplets and are compared with previous values. Notation $a \pm b$ means $a \times 10^{b}$, "O" means for others and "P" for present. The last line of a set of dipole allowed transitions corresponds to the LS multiplet

\begin{tabular}{|c|c|c|c|c|c|c|c|c|c|}
\hline$C_{i}$ & $C_{j}$ & $S_{i} L_{i} \pi_{i}$ & $S_{j} L_{j} \pi_{j}$ & $g_{i}\left(I_{i}\right)$ & $g_{j}\left(I_{j}\right)$ & $f_{i j}(O)$ & $f_{i j}(P)$ & $A_{j i}\left(\mathrm{~s}^{-1}, O\right)$ & $A_{j i}\left(\mathrm{~s}^{-1}, P\right)$ \\
\hline $\begin{array}{l}2 \mathrm{~s} 22 \mathrm{p} 2 \\
2 \mathrm{~s} 22 \mathrm{p} 2 \\
2 \mathrm{~s} 22 \mathrm{p} 2 \\
2 \mathrm{~s} 22 \mathrm{p} 2 \\
2 \mathrm{~s} 22 \mathrm{p} 2 \\
2 \mathrm{~s} 22 \mathrm{p} 2 \\
2 \mathrm{~s} 22 \mathrm{p} 2\end{array}$ & $\begin{array}{l}-2 \mathrm{~s} 2 \mathrm{p} 3 \\
-2 \mathrm{~s} 2 \mathrm{p} 3 \\
-2 \mathrm{~s} 2 \mathrm{p} 3 \\
-2 \mathrm{~s} 2 \mathrm{p} 3 \\
-2 \mathrm{~s} 2 \mathrm{p} 3 \\
-2 \mathrm{~s} 2 \mathrm{p} 3 \\
-2 \mathrm{~s} 2 \mathrm{p} 3\end{array}$ & $\begin{array}{l}3 \mathrm{P} 0 \\
\text { 3P0 } \\
\text { 3P0 } \\
\text { 3P0 } \\
\text { 3P0 } \\
\text { 3P0 } \\
\text { 3P0 }\end{array}$ & $\begin{array}{l}{ }^{3} \mathrm{D} 1 \\
{ }^{3} \mathrm{D} 1 \\
{ }^{3} \mathrm{D} 1 \\
{ }^{3} \mathrm{D} 1 \\
{ }^{3} \mathrm{D} 1 \\
{ }^{3} \mathrm{D} 1 \\
{ }^{3} \mathrm{D} 1\end{array}$ & $\begin{array}{l}11(1) \\
3(1) \\
3(1) \\
5(1) \\
5(1) \\
5(1) \\
9\end{array}$ & $\begin{array}{l}3(1) \\
3 \\
5(1) \\
3 \\
5(1) \\
7(2) \\
7(1) \\
15\end{array}$ & $\begin{array}{l}9.3-2^{1} \\
2.4-3^{1} \\
5.1-2^{1} \\
1.5-4^{1} \\
4.4-5^{1} \\
2.95-2^{1} \\
4.5-2^{1}, 3.71-2^{2}\end{array}$ & $\begin{array}{l}8.49-2 \\
2.45-3 \\
4.62-2 \\
5.02-3 \\
7.64-6 \\
2.70-2 \\
4.20-2\end{array}$ & & \\
\hline $\begin{array}{l}2 \mathrm{~s} 22 \mathrm{p} 2 \\
2 \mathrm{~s} 22 \mathrm{p} 2 \\
2 \mathrm{~s} 22 \mathrm{p} 2 \\
2 \mathrm{~s} 22 \mathrm{p} 2 \\
2 \mathrm{~s} 22 \mathrm{p} 2 \\
2 \mathrm{~s} 22 \mathrm{p} 2 \\
2 \mathrm{~s} 22 \mathrm{p} 2\end{array}$ & $\begin{array}{l}-2 \mathrm{~s} 2 \mathrm{p} 3 \\
-2 \mathrm{~s} 2 \mathrm{p} 3 \\
-2 \mathrm{~s} 2 \mathrm{p} 3 \\
-2 \mathrm{~s} 2 \mathrm{p} 3 \\
-2 \mathrm{~s} 2 \mathrm{p} 3 \\
-2 \mathrm{~s} 2 \mathrm{p} 3 \\
-2 \mathrm{~s} 2 \mathrm{p} 3\end{array}$ & $\begin{array}{l}3 \mathrm{P} 0 \\
\text { 3P0 } \\
3 \mathrm{P} 0 \\
\text { 3P0 } \\
\text { 3P0 } \\
\text { 3P0 } \\
\text { 3P0 }\end{array}$ & $\begin{array}{l}{ }^{3} \mathrm{P} 1 \\
{ }^{3} \mathrm{P} 1 \\
{ }^{3} \mathrm{P} 1 \\
{ }^{3} \mathrm{P} 1 \\
{ }^{3} \mathrm{P} 1 \\
{ }^{3} \mathrm{P} 1 \\
{ }^{3} \mathrm{P} 1\end{array}$ & $\begin{aligned} & 1(1) \\
& 3(1) \\
& 3(1) \\
& 3(1) \\
& 5(1) \\
& 5(1) \\
& 9\end{aligned}$ & $\begin{array}{l}3(2) \\
11(1) \\
33(2) \\
5(3) \\
3(2) \\
5(3) \\
9\end{array}$ & $\begin{array}{l}2.25-2^{1} \\
1.70-2^{1} \\
3.54-2^{1} \\
1.2-3^{1} \\
4.4-3^{1} \\
4.79-2^{1} \\
4.98-2^{1}, 4.09-2^{2}\end{array}$ & $\begin{array}{l}2.25-2 \\
1.54-2 \\
3.23-2 \\
1.38-3 \\
1.13-2 \\
4.46-2 \\
5.03-2\end{array}$ & & \\
\hline $\begin{array}{l}2 \mathrm{~s} 22 \mathrm{p} 2 \\
2 \mathrm{~s} 22 \mathrm{p} 2 \\
2 \mathrm{~s} 22 \mathrm{p} 2 \\
2 \mathrm{~s} 22 \mathrm{p} 2\end{array}$ & $\begin{array}{l}-2 \mathrm{~s} 2 \mathrm{p} 3 \\
-2 \mathrm{~s} 2 \mathrm{p} 3 \\
-2 \mathrm{~s} 2 \mathrm{p} 3 \\
-2 \mathrm{~s} 2 \mathrm{p} 3\end{array}$ & $\begin{array}{l}3 \mathrm{P} 0 \\
3 \mathrm{P} 0 \\
3 \mathrm{P} 0 \\
3 \mathrm{P} 0\end{array}$ & $\begin{array}{l}{ }^{3} \mathrm{~S} 1 \\
{ }^{3} \mathrm{~S} 1 \\
{ }^{3} \mathrm{~S} 1 \\
{ }^{3} \mathrm{~S} 1\end{array}$ & $\begin{array}{r}1 \\
33(1) \\
5(1) \\
9\end{array}$ & $\begin{array}{r}3(3) \\
3(3) \\
3(3) \\
3\end{array}$ & $\begin{array}{l}3.70-2^{1} \\
3.79-2^{1} \\
6.0-2^{1} \\
5.1-2^{1}, 4.68-2^{2}\end{array}$ & $\begin{array}{l}3.58-2 \\
3.67-2 \\
4.01-2 \\
3.86-2\end{array}$ & & \\
\hline $\begin{array}{l}2 \mathrm{~s} 22 \mathrm{p} 2 \\
2 \mathrm{~s} 22 \mathrm{p} 2\end{array}$ & $\begin{array}{l}-2 \mathrm{~s} 2 \mathrm{p} 3 \\
-2 \mathrm{~s} 2 \mathrm{p} 3\end{array}$ & $\begin{array}{l}3 \mathrm{P} 0 \\
3 \mathrm{P} 0\end{array}$ & $\begin{array}{l}{ }^{1} \mathrm{D} 1 \\
{ }^{1} \mathrm{D} 1\end{array}$ & $\begin{array}{l}3(1) \\
5(1)\end{array}$ & $\begin{array}{l}5(4) \\
5(4)\end{array}$ & $\begin{array}{l}9.5-4^{1} \\
1.3-2^{1}\end{array}$ & $\begin{array}{l}9.38-4 \\
1.20-2\end{array}$ & & \\
\hline $\begin{array}{l}2 \mathrm{~s} 22 \mathrm{p} 2 \\
2 \mathrm{~s} 22 \mathrm{p} 2 \\
2 \mathrm{~s} 22 \mathrm{p} 2\end{array}$ & $\begin{array}{l}-2 \mathrm{~s} 2 \mathrm{p} 3 \\
-2 \mathrm{~s} 2 \mathrm{p} 3 \\
-2 \mathrm{~s} 2 \mathrm{p} 3\end{array}$ & $\begin{array}{l}3 \mathrm{P} 0 \\
3 \mathrm{P} 0 \\
3 \mathrm{P} 0\end{array}$ & $\begin{array}{l}{ }^{1} \mathrm{P} 1 \\
{ }^{1} \mathrm{P} 1 \\
{ }^{1} \mathrm{P} 1\end{array}$ & $\begin{array}{l}1(1) \\
3(1) \\
5(1)\end{array}$ & $\begin{array}{l}3(4) \\
3(4) \\
3(4)\end{array}$ & $\begin{array}{l}5.6-3^{1} \\
1.6-4^{1}\end{array}$ & $\begin{array}{l}1.03-4 \\
5.29-3 \\
8.45-4\end{array}$ & & \\
\hline $\begin{array}{l}2 \mathrm{~s} 22 \mathrm{p} 2 \\
2 \mathrm{~s} 22 \mathrm{p} 2 \\
2 \mathrm{~s} 22 \mathrm{p} 2\end{array}$ & $\begin{array}{l}-2 \mathrm{~s} 2 \mathrm{p} 3 \\
-2 \mathrm{~s} 2 \mathrm{p} 3 \\
-2 \mathrm{~s} 2 \mathrm{p} 3\end{array}$ & $\begin{array}{l}{ }^{1} \mathrm{D} 0 \\
{ }^{1} \mathrm{D} 0 \\
{ }^{1} \mathrm{D} 0\end{array}$ & $\begin{array}{l}{ }^{3} \mathrm{D} 1 \\
{ }^{3} \mathrm{D} 1 \\
{ }^{3} \mathrm{D} 1\end{array}$ & $\begin{array}{l}5(2) \\
5(2) \\
5(2)\end{array}$ & $\begin{array}{l}33(1) \\
5(2) \\
7(1)\end{array}$ & $\begin{array}{l}6.4-4^{1} \\
2.0-4^{1} \\
7.0-3^{1}\end{array}$ & $\begin{array}{l}2.96-5 \\
2.27-4 \\
6.59-3\end{array}$ & & \\
\hline $2 \mathrm{~s} 22 \mathrm{p} 2$ & $-2 \mathrm{~s} 2 \mathrm{p} 3$ & ${ }^{1} \mathrm{D} 0$ & ${ }^{1} \mathrm{P} 1$ & $5(2)$ & $3(4)$ & $6.2-2^{1}, 5.36-2^{2}$ & $5.30-2$ & & \\
\hline $2 \mathrm{~s} 22 \mathrm{p} 2$ & $-2 \mathrm{~s} 2 \mathrm{p} 3$ & ${ }^{1} \mathrm{D} 0$ & ${ }^{1} \mathrm{D} 1$ & 5 & 5 & $9.2-2^{1}, 9.14-2^{2}$ & $8.56-2$ & & \\
\hline $2 \mathrm{~s} 22 \mathrm{p} 2$ & $-2 \mathrm{~s} 2 \mathrm{p} 3$ & ${ }^{1} \mathrm{~S} 0$ & ${ }^{1} \mathrm{P} 1$ & $1(2)$ & $3(4)$ & $1.04-1^{2}$ & $9.79-2$ & & \\
\hline $2 \mathrm{~s} 22 \mathrm{p} 2$ & $-2 \mathrm{~s} 2 \mathrm{p} 3$ & ${ }^{1} \mathrm{~S} 0$ & ${ }^{3} \mathrm{D} 1$ & $1(2)$ & $3(1)$ & $1.5-3^{1}$ & $1.07-3$ & & \\
\hline $2 \mathrm{~s} 22 \mathrm{p} 2$ & $-2 \mathrm{~s} 2 \mathrm{p} 3$ & ${ }^{1} \mathrm{~S} 0$ & ${ }^{3} \mathrm{P} 1$ & $1(2)$ & $3(2)$ & $2.4-3^{1}$ & $2.21-3$ & & \\
\hline $2 \mathrm{~s} 22 \mathrm{p} 2$ & $-2 \mathrm{~s} 2 \mathrm{p} 3$ & ${ }^{1} \mathrm{~S} 0$ & ${ }^{3} \mathrm{~S} 1$ & $1(2)$ & $3(3)$ & $5.9-3^{1}$ & $5.57-3$ & & \\
\hline $\begin{array}{l}2 \mathrm{~s} 2 \mathrm{p} 3 \\
2 \mathrm{~s} 2 \mathrm{p} 3\end{array}$ & $\begin{array}{l}-2 \mathrm{p} 4 \\
-2 \mathrm{p} 4\end{array}$ & $\begin{array}{l}{ }^{3} \mathrm{P} 1 \\
{ }^{3} \mathrm{P} 1\end{array}$ & $\begin{array}{l}{ }^{1} \mathrm{D} 0 \\
{ }^{1} \mathrm{D} 0\end{array}$ & $\begin{array}{l}3(2) \\
5(3)\end{array}$ & $\begin{array}{l}5(4) \\
5(4)\end{array}$ & $\begin{array}{l}4.1-3^{1} \\
4.9-3^{1}\end{array}$ & $\begin{array}{l}7.81-5 \\
4.79-3\end{array}$ & & \\
\hline $\begin{array}{l}2 \mathrm{~s} 2 \mathrm{p} 3 \\
2 \mathrm{~s} 2 \mathrm{p} 3\end{array}$ & $\begin{array}{l}-2 \mathrm{p} 4 \\
-2 \mathrm{p} 4\end{array}$ & $\begin{array}{l}{ }^{3} \mathrm{D} 1 \\
{ }^{3} \mathrm{D} 1\end{array}$ & $\begin{array}{l}{ }^{1} \mathrm{D} 0 \\
{ }^{1} \mathrm{D} 0\end{array}$ & $\begin{array}{l}5(2) \\
7(1)\end{array}$ & $\begin{array}{l}5(4) \\
5(4)\end{array}$ & $\begin{array}{l}1.0-3^{1} \\
6.2-3^{1}\end{array}$ & $\begin{array}{l}1.08-3 \\
5.96-3\end{array}$ & & \\
\hline $\begin{array}{l}2 \mathrm{~s} 2 \mathrm{p} 3 \\
2 \mathrm{~s} 2 \mathrm{p} 3 \\
2 \mathrm{~s} 2 \mathrm{p} 3 \\
2 \mathrm{~s} 2 \mathrm{p} 3\end{array}$ & $\begin{array}{l}-2 \mathrm{p} 4 \\
-2 \mathrm{p} 4 \\
-2 \mathrm{p} 4 \\
-2 \mathrm{p} 4\end{array}$ & $\begin{array}{l}{ }^{3} \mathrm{~S} 1 \\
{ }^{3} \mathrm{~S} 1 \\
{ }^{3} \mathrm{~S} 1 \\
{ }^{3} \mathrm{~S} 1\end{array}$ & $\begin{array}{l}{ }^{3} \mathrm{P} 0 \\
{ }^{3} \mathrm{P} 0 \\
{ }^{3} \mathrm{P} 0 \\
{ }^{3} \mathrm{P} 0\end{array}$ & $\begin{array}{r}3(3) \\
3(3) \\
3(3) \\
3\end{array}$ & $\begin{array}{r}1(3) \\
3(2) \\
5(3) \\
9\end{array}$ & $\begin{array}{l}2.35-2^{1} \\
5.2-2^{1} \\
5.6-2^{1} \\
0.13^{1}, 0.112^{2}\end{array}$ & $\begin{array}{l}2.10-2 \\
4.64-2 \\
6.60-2 \\
0.133\end{array}$ & & \\
\hline $\begin{array}{l}2 \mathrm{~s} 2 \mathrm{p} 3 \\
2 \mathrm{~s} 2 \mathrm{p} 3 \\
2 \mathrm{~s} 2 \mathrm{p} 3 \\
2 \mathrm{~s} 2 \mathrm{p} 3 \\
2 \mathrm{~s} 2 \mathrm{p} 3 \\
2 \mathrm{~s} 2 \mathrm{p} 3 \\
2 \mathrm{~s} 2 \mathrm{p} 3\end{array}$ & $\begin{array}{l}-2 \mathrm{p} 4 \\
-2 \mathrm{p} 4 \\
-2 \mathrm{p} 4 \\
-2 \mathrm{p} 4 \\
-2 \mathrm{p} 4 \\
-2 \mathrm{p} 4 \\
-2 \mathrm{p} 4\end{array}$ & $\begin{array}{l}{ }^{3} \mathrm{P} 1 \\
{ }^{3} \mathrm{P} 1 \\
{ }^{3} \mathrm{P} 1 \\
{ }^{3} \mathrm{P} 1 \\
{ }^{3} \mathrm{P} 1 \\
{ }^{3} \mathrm{P} 1 \\
{ }^{3} \mathrm{P} 1\end{array}$ & $\begin{array}{l}{ }^{3} \mathrm{P} 0 \\
{ }^{3} \mathrm{P} 0 \\
{ }^{3} \mathrm{P} 0 \\
{ }^{3} \mathrm{P} 0 \\
{ }^{3} \mathrm{P} 0 \\
{ }^{3} \mathrm{P} 0 \\
{ }^{3} \mathrm{P} 0\end{array}$ & $\begin{array}{r}3(2) \\
1(1) \\
3(2) \\
5(3) \\
3(2) \\
5(3) \\
9\end{array}$ & $\begin{array}{r}1(3) \\
3(2) \\
3(2) \\
3(2) \\
5(3) \\
5(3) \\
9\end{array}$ & $\begin{array}{l}1.55-2^{1} \\
3.41-2^{1} \\
3.4-4^{1} \\
2.50-2^{1} \\
1.84-2^{1} \\
1.11-2^{1} \\
3.6-2^{1}, 2.62-2^{2}\end{array}$ & $\begin{array}{l}1.38-2 \\
3.12-2 \\
1.92-4 \\
2.22-2 \\
2.68-3 \\
1.12-2 \\
2.70-2\end{array}$ & & \\
\hline $\begin{array}{l}2 \mathrm{~s} 2 \mathrm{p} 3 \\
2 \mathrm{~s} 2 \mathrm{p} 3 \\
2 \mathrm{~s} 2 \mathrm{p} 3 \\
2 \mathrm{~s} 2 \mathrm{p} 3 \\
2 \mathrm{~s} 2 \mathrm{p} 3 \\
2 \mathrm{~s} 2 \mathrm{p} 3 \\
2 \mathrm{~s} 2 \mathrm{p} 3\end{array}$ & $\begin{array}{l}-2 \mathrm{p} 4 \\
-2 \mathrm{p} 4 \\
-2 \mathrm{p} 4 \\
-2 \mathrm{p} 4 \\
-2 \mathrm{p} 4 \\
-2 \mathrm{p} 4 \\
-2 \mathrm{p} 4\end{array}$ & $\begin{array}{l}\text { 3D1 } \\
\text { 3D1 } \\
\text { 3D1 } \\
\text { 3D1 } \\
\text { 3D1 } \\
\text { 3D1 } \\
\text { 3D1 }\end{array}$ & $\begin{array}{l}{ }^{3} \mathrm{P} 0 \\
{ }^{3} \mathrm{P} 0 \\
{ }^{3} \mathrm{P} 0 \\
{ }^{3} \mathrm{P} 0 \\
{ }^{3} \mathrm{P} 0 \\
{ }^{3} \mathrm{P} 0 \\
{ }^{3} \mathrm{P} 0\end{array}$ & $\begin{array}{l}3(1) \\
3(1) \\
5(2) \\
3(1) \\
5(2) \\
7(1) \\
15\end{array}$ & $\begin{array}{r}1(3) \\
3(2) \\
3(2) \\
5(3) \\
5(3) \\
5(3) \\
9\end{array}$ & $\begin{array}{l}2.03-2^{1} \\
2.52-2^{1} \\
2.20-2^{1} \\
1.25-2^{1} \\
2.92-2^{1} \\
4.67-2^{1} \\
5.1-2^{1}, 4.69-2^{2}\end{array}$ & $\begin{array}{l}1.91-2 \\
2.32-2 \\
2.09-2 \\
8.03-4 \\
2.62-2 \\
4.23-2 \\
4.43-2\end{array}$ & & \\
\hline $\begin{array}{l}2 \mathrm{~s} 2 \mathrm{p} 3 \\
2 \mathrm{~s} 2 \mathrm{p} 3 \\
2 \mathrm{~s} 2 \mathrm{p} 3\end{array}$ & $\begin{array}{l}-2 \mathrm{p} 4 \\
-2 \mathrm{p} 4 \\
-2 \mathrm{p} 4\end{array}$ & $\begin{array}{l}{ }^{1} \mathrm{P} 1 \\
{ }^{1} \mathrm{P} 1 \\
{ }^{1} \mathrm{P} 1\end{array}$ & $\begin{array}{l}{ }^{3} \mathrm{P} 0 \\
{ }^{3} \mathrm{P} 0 \\
{ }^{3} \mathrm{P} 0\end{array}$ & $\begin{array}{l}3(4) \\
3(4) \\
3(4)\end{array}$ & $\begin{array}{l}1(3) \\
3(2) \\
5(3)\end{array}$ & $\begin{array}{l}4.8-3^{1} \\
1.9-3^{1}\end{array}$ & $\begin{array}{l}3.18-5 \\
4.18-3 \\
2.31-3\end{array}$ & & \\
\hline $\begin{array}{l}2 \mathrm{~s} 22 \mathrm{p} 2 \\
2 \mathrm{~s} 22 \mathrm{p} 2\end{array}$ & $\begin{array}{l}-2 \mathrm{~s} 2 \mathrm{p} 3 \\
-2 \mathrm{~s} 2 \mathrm{p} 3\end{array}$ & $\begin{array}{l}3 \mathrm{P} 0 \\
3 \mathrm{P} 0\end{array}$ & $\begin{array}{l}{ }^{5} \mathrm{~S} 1 \\
{ }^{5} \mathrm{~S} 1\end{array}$ & $\begin{array}{l}3(1) \\
5(1)\end{array}$ & $\begin{array}{l}5(1) \\
5(1)\end{array}$ & $\begin{array}{l}5.3-4^{1} \\
3.8-4^{1}\end{array}$ & $\begin{array}{l}5.155-4 \\
3.72-4\end{array}$ & $\begin{array}{l}3.61+7^{3} \\
3.27+7^{3}\end{array}$ & $\begin{array}{l}3.51+7 \\
3.39+7\end{array}$ \\
\hline $2 \mathrm{~s} 22 \mathrm{p} 2$ & $-2 \mathrm{~s} 2 \mathrm{p} 3$ & ${ }^{1} \mathrm{D} 0$ & ${ }^{5} \mathrm{~S} 1$ & 5 & 2 & & $3.53-5$ & $1.46+6^{3}$ & $1.38+6$ \\
\hline
\end{tabular}

\footnotetext{
1 Cheng et al. (1979), ${ }^{2}$ Luo \& Pradhan (1989), ${ }^{3}$ Mendoza et al. (1999).
} 
Table 9. Level indices of the calculated fine structure energy levels for various $J \pi$ symmetries that have been observed and among which the allowed transitions have been reprocessed using the observed energies. $n_{j}$ is the number of observed levels in each symmetry

\begin{tabular}{|c|c|c|}
\hline$J \pi$ & $n_{j}$ & level indices \\
\hline \multicolumn{3}{|r|}{ Ar XIII } \\
\hline $0^{\mathrm{e}}$ & 3 & $1,2,3$ \\
\hline $0^{\circ}$ & 6 & $1,2,3,8,12,18$ \\
\hline $1^{\mathrm{e}}$ & 2 & 1,2 \\
\hline $1^{\mathrm{o}}$ & 17 & $1,2,3,4,5,6,7,8,9,16,18,20,21,30,31,43,45$ \\
\hline $2^{\mathrm{e}}$ & 5 & $1,2,3,4,14$ \\
\hline $2^{\mathrm{o}}$ & 15 & $1,2,3,4,5,6,7,8,9,15,16,19,29,44,46$ \\
\hline $3^{\mathrm{e}}$ & 1 & 6 \\
\hline $3^{\circ}$ & 5 & $1,2,3,4,8$ \\
\hline $4^{\circ}$ & 1 & 1 \\
\hline \multicolumn{3}{|r|}{ Fe XXI } \\
\hline $0^{\mathrm{e}}$ & 4 & $1,2,3,4$ \\
\hline $0^{\circ}$ & 1 & 1 \\
\hline $1^{\mathrm{e}}$ & 2 & 1,2 \\
\hline $1^{\mathrm{o}}$ & 8 & $1,2,3,4,11,39,40,67$ \\
\hline $2^{\mathrm{e}}$ & 4 & $1,2,3,4$ \\
\hline $2^{\circ}$ & 11 & $1,2,3,4,6,8,9,39,40,41,72$ \\
\hline $3^{\circ}$ & 9 & $1,2,3,4,22,23,24,50,51$ \\
\hline
\end{tabular}

contrast to the large complete files (Tables 5 and 6 ) they are given with complete identification. The level index, $I_{i}$, for each energy level in the tables is given next to the $J$-value for an easy access to the complete $f$-file. For example, the transition $J=0^{\mathrm{e}}\left(I_{i}=1\right) \rightarrow J=1^{\circ}\left(I_{j}=1\right)$ in Table 7a corresponds to the first transition in Table 5, and hence, the $f$ - and $A$-values of Table 7a should replace those in Table 5 . The overall replacement in Tables 5 or 6 (as may be needed in a model calculation requring large number of transitions) can be carried out by sorting out the reprocessed transitions through their energy level indices. The set of level indices of each symmetry $J \pi$ for both Ar XIII and Fe XXI that correspond to these transitions are given in Table 9.

The reprocessed transitions are further ordered in terms of their configurations and $L S$ terms. This enables one to obtain the $f$-values for the $L S$ multiplets and check the completeness of the set of fine structure components belonging to the multiplet. However, the completeness depends also on the observed set of fine structure levels since the transitions correspond only to the observed levels. The $L S$ multiplets are useful for various comparisons with other calculations and experiment where fine structure transitions can not be resolved. A partial set of these transitions is presented in Table 7b for Ar XIII and Table $8 \mathrm{~b}$ for Fe XXI (the complete table is available electronically).

The oscillator strengths are compared with other calculations in Tables 7b for Ar XIII and 8b for Fe XXI. The oscillator strengths for a large number of fine structure transitions in Ar XIII and other carbon like ions have been obtained by Zhang \& Sampson (1997) in the relativistic distorted wave calculations. They consider the transitions among the $n=2$ and $n=3$ levels. The other source of transition probabilities for Ar XIII is TOPbase (the database for the Opacity Project data) where transition probabilties for $L S$ multiplets are given for states with $n \leq 10$. These values were calculated by Luo \& Pradhan (1989) in the close coupling approximation using non-relativistic R-matrix method. Comparison of the present BPRM oscillator strengths with the previous calculations in Table $7 \mathrm{~b}$ shows that present $L S$ multiplets obtained from the fine structure components agree quite well with those of Luo and Pradhan except for the transition, $2 \mathrm{~s} 2 \mathrm{p}^{3}\left({ }^{3} \mathrm{D}^{\circ}\right)-2 \mathrm{p}^{4}\left({ }^{3} \mathrm{P}\right)$. However, comparison with Zhang and Sampson shows various degrees of agreement. There is good agreement for some fine structure components compared to others in the same $L S$ multiplet, e.g. for $2 \mathrm{~s}^{2} 2 \mathrm{p}^{2}\left({ }^{3} \mathrm{P}\right)-2 \mathrm{~s} 2 \mathrm{p}^{3}\left({ }^{3} \mathrm{D}^{\circ}\right)$. The largest difference is found in the weak transitions.

The $f$-values of Fe XXI have been compiled by Fuhr et al. (1988) and Shirai et al. (1990) (both references present the same values). They report mainly the multiconfiguration Dirac-Fock calculations by Cheng et al. (1979). The other source of large amount of data in $L S$ coupling is from TOPbase (Cunto et al. 1993) calculated by Luo \& Pradhan (1989) under the Opacity Project. Similar to the above case of Ar XIII, the Fe XXI oscillator strengths compare well for some but poorly for other fine structure components within a multiplet of previous calculations (Table 8b). For example, most of the fine structure components of the dipole allowed multiplets, $2 \mathrm{~s}^{2} 2 \mathrm{p}^{2}\left({ }^{3} \mathrm{P}\right)-2 \mathrm{~s} 2 \mathrm{p}^{3}\left({ }^{3} \mathrm{D}^{\mathrm{o}},{ }^{3} \mathrm{P}^{\mathrm{o}},{ }^{3} \mathrm{~S}^{\mathrm{o}}\right)$, have comparable values from the two calculations, BPRM and Dirac-Fock, except for the weak transitions. While BPRM $f$-values agree well with those by Cheng et al. for the intercombination transitions, 5-5 and 5-7 of $2 \mathrm{~s}^{2} 2 \mathrm{p}^{2}\left({ }^{1} \mathrm{D}\right)-2 \mathrm{~s} 2 \mathrm{p}^{3}\left({ }^{3} \mathrm{D}^{\circ}\right)$, they differ considerably for the transition $5-3$. The $L S$ multiplets are compared with those of Luo \& Pradhan (1989) and in general agree very well with the present BPRM $A$-values.

Recently Mendoza et al. (1999) have calculated the transition probabilties for the intercombination transitions $2 \mathrm{~s} 2 \mathrm{p}^{3}\left({ }^{5} \mathrm{~S}_{2}^{\circ}\right)-2 \mathrm{~s}^{2} 2 \mathrm{p}^{2}\left({ }^{3} \mathrm{P}_{1,2},{ }^{1} \mathrm{D}_{2}\right)$ for the carbon isoelectronic sequence ions. Through extensive relativistic atomic structure calculations they study the effects of Breit interaction in the transition probabilties for these weak transitions. They estimate an accuracy better than $10 \%$ for $A$-values belonging to the ground levels ${ }^{3} \mathrm{P}_{1,2}$ and $20 \%$ for the ${ }^{1} \mathrm{D}_{2}$. Their values for Ar XIII agree very well with the present BPRM $A$-values (Table $7 \mathrm{~b}$ ), especially those to the ground levels; it is $4 \%$ for the ${ }^{5} S_{2}^{o}-{ }^{3} P_{1}$ and $0.01 \%$ for the ${ }^{5} \mathrm{~S}_{2}^{\circ}-{ }^{3} \mathrm{P}_{2}$ transitions. The agreement is $14 \%$ for the ${ }^{5} \mathrm{~S}_{2}^{\circ}-{ }^{1} \mathrm{D}_{2}$ for which they assign a larger uncertainty. For Fe XXI, present $f$-values for transitions to the ground configuration levels $2 \mathrm{~s} 2 \mathrm{p}^{3}\left({ }^{5} \mathrm{~S}^{\circ}\right)-2 \mathrm{~s}^{2} 2 \mathrm{p}^{2}\left({ }^{3} \mathrm{P}_{1,2},{ }^{1} \mathrm{D}_{2}\right)$ agree very well with 
those by Cheng et al. The agreement is also quite good with the $A$-values obtained in elaborate calculations by Mendoza et al. (1999); about $4 \%$ for both the transitions to the ground term, ${ }^{5} \mathrm{~S}_{2}^{\circ}-{ }^{3} \mathrm{P}_{1,2}$, and $5 \%$ for the ${ }^{5} \mathrm{~S}_{2}^{\mathrm{o}}-{ }^{1} \mathrm{D}_{2}$ transition.

Comparison of the present transition probabilities for the intercombination transitions with those of Mendoza et al. (1999) provides a measure of uncertainty of the present BPRM results. They studied the effect of Breit spin-spin and spin-other orbit interactions which are not included in the present work. The good agreement with them for these very weak transtions indicate the uncertainty of the present results to a maximum of $14 \%$ for most of the transitions. The discrepancy between the oscillator strengths in length and velocity forms $\left(f_{\mathrm{L}}\right.$ and $f_{\mathrm{V}}$ ) is an indicator of accuracy consistent with the method of calculations. In the present results, it is found that the dispersion of the $f_{\mathrm{L}}$ and $f_{\mathrm{V}}$ values for Fe XXI is mainly in the very weak transitons. However, the dispersion is larger for Ar XIII; it is expected that the dispersion is caused mainly by the values of $f_{\mathrm{V}}$. Inclusion of more configurations in the Ar XIII wavefunction expansion could have improved the agreement; $n=3$ configurations are still important and should be included in the target as spectroscopic configurations. However, that is computationally intractable in the BPRM calculations. It may be noted that the length form in a close coupling approximation is more accurate than the velocity form as it depends more on the asymptotic form of the wavefunction which is better represented in the R-matrix calculations.

\section{Conclusion}

We have described some of the first large-scale ab initio relativistic calculations of transition probabilities now in progress under the Iron Project. Large datasets for the fine structure energy levels and oscillator strengths for the carbon like Ar XIII and Fe XXI are presented and the amount exceeds considerably the presently available data. All the calculated energy levels, 1274 for Ar XIII and 1611 for Fe XXI, have been spectroscopically identified and checked for completeness. All data are available electronically. Part of the $f$-values have been reprocessed using available observed energies for better accuracy.
Acknowledgements. This work was partially supported by U.S. National Science Foundation (AST-9870089) and NASA (NAG5-7903). The computational work was carried out on the Cray T94 at the Ohio Supercomputer Center in Columbus, Ohio.

\section{References}

Berrington K.A., Eissner W.B., Norrington P.H., 1995, Comput. Phys. Commun. 92, 290

Cheng K.T., Kim Y.-K., Desclaux J.P., 1979, At. Data Nucl. Data Tables 24, 111

Cunto W., Mendoza C., Ochsenbein F., Zeippen C.J., 1993, A\&A 275, L5

Eissner W., Jones M., Nussbaumer H., 1974, Comput. Phys. Commun. 8, 270

Fuhr J.R., Martin G.A., Wiese W.L., 1988, J. Phys. Chem. Ref. Data 17, Suppl. 4

Hummer D.G., Berrington K.A., Eissner W., 1993, A\&A 279, 298 (IP)

Kelly R.L. (unpublished), data obtained from the NIST

Luo D., Pradhan A.K., 1989, J. Phys. B 22, 3377

Mendoza C., Zeippen C.J., Storey P.J., 1999, A\&AS 135, 159 (IP.XXXIII)

Nahar S.N., 1995, A\&A 293, 967 (IP.VII)

Nahar S.N., 1999, At. Data Nucl. Data Tables 72, 129

Nahar S.N., Delahaye F., Pradhan A.K., Zeippen C.J., 2000, A\&AS 144, 141

Nahar S.N., Pradhan A.K., 1996, A\&AS 119, 509 (IP.XVII)

Nahar S.N., Pradhan A.K., 1999, A\&AS 135, 347 (IP.XXXV)

Nahar S.N., Pradhan A.K., 2000, Phys. Scr. 61, 675

Pallavicini R., Serio S., Vaiana G.S., 1977, ApJ 223, 1058

Scott N.S., Burke P.G., 1980, J. Phys. B 12, 4299

Scott N.S., Taylor K.T., 1982, Comput. Phys. Commun. 25, 347

Seaton M.J., 1987, J. Phys. B 20, 6363

Seaton M.J., Yan Y., Mihalas D., Pradhan A.K., 1994, MNRAS 266, 805

Shirai T., Funatake Y., Mori J., Sugar J., Wiese W.L., Nakai Y., 1990, J. Phys. Chem. Ref. Data 19, 127

Sugar J., Corliss C., 1985, J. Phys. Chem. Ref. Data 14, Suppl.

The Opacity Project $1 \& 2$, compiled by the Opacity Project team, Institute of Physics, London, UK, 1995, 1996 (OP)

Zhang H.L., Sampson D.H., 1997, At. Data Nucl. Data Tables 65, 183 